\title{
Supply Voltage Dependence of Heavy Ion Induced SEEs on 65nm CMOS Bulk SRAMs
}

\author{
A Thesis Submitted to the College of \\ Graduate Studies and Research \\ In Partial Fulfillment of the Requirements \\ For the Degree of Master of Science \\ In the Department of Electrical and Computer Engineering \\ University of Saskatchewan \\ Saskatoon
} By QIONG WU

(C) Copyright Qiong Wu, June, 2015. All rights reserved. 


\section{PERMISSION TO USE}

In presenting this thesis in partial fulfilment of the requirements for a Postgraduate degree from the University of Saskatchewan, I agree that the Libraries of this University may make it freely available for inspection. I further agree that permission for copying of this thesis in any manner, in whole or in part, for scholarly purposes may be granted by the professor or professors who supervised my thesis work or, in their absence, by the Head of the Department or the Dean of the College in which my thesis work was done. It is understood that any copying or publication or use of this thesis or parts thereof for financial gain shall not be allowed without my written permission. It is also understood that due recognition shall be given to me and to the University of Saskatchewan in any scholarly use which may be made of any material in my thesis.

Requests for permission to copy or to make other use of material in this thesis in whole or part should be addressed to:

Head of the Department of Electrical and Computer Engineering

57 Campus Drive

University of Saskatchewan

Saskatoon, Saskatchewan

Canada

S7N 5A9 


\section{ACKNOWLEDGEMENT}

None of my accomplishments would be possible without the support and assistance of numerous individuals. First of all, I would like to thank Dr. Li Chen, my supervisor during my postgraduate studies at University of Saskatchewan. Dr. Chen has been incredibly helpful and truly an inspiration on both professional and personal levels. He was always there to lend advice when I asked and to give motivation when I needed it. Additionally, financial assistance provided by the Dr. Li Chen and University of Saskatchewan through research funding and Graduate Scholarship is thankfully acknowledged.

Thank you to my fellow graduate students, with whom I have spent so much time. I also want to express my sincere gratitude to Rui Liu and Yuanqing Li for all of their help with chip fabrication and data analysis. Thank you to everyone else past and present in room 2B60 and 3C80 of Engineering Building. Also, I would like to acknowledge the staff and faculty of the Electrical and Computer Engineering Department for their support. They all have helped me in different ways over the course of this work.

Lastly and with the most gravity, I would like to express my deepest gratitude to my husband. I am grateful to my parents, for their unselfish love and constant support. They have encouraged my education, instilled honesty, and nurtured a predilection to science. 


\begin{abstract}
The power consumption of Static Random Access Memory (SRAM) has become an important issue for modern integrated circuit design, considering the fact that they occupy large area and consume significant portion of power consumption in modern nanometer chips. SRAM operating in low power supply voltages has become an effective approach in reducing power consumption. Therefore, it is essential to experimentally characterize the single event effects (SEE) of hardened and unhardened SRAM cells to determine their appropriate applications, especially when a low supply voltage is preferred. In this thesis, a SRAM test chip was designed and fabricated with four cell arrays sharing the same peripheral circuits, including two types of unhardened cells (standard 6T and sub-threshold 10T) and two types of hardened cells (Quatro and DICE). The systems for functional and radiation tests were built up with power supply voltages that ranged from near threshold $0.4 \mathrm{~V}$ to normal supply 1 V. The test chip was irradiated with alpha particles and heavy ions with various linear energy transfers (LETs) at different core supply voltages, ranging from $1 \mathrm{~V}$ to $0.4 \mathrm{~V}$. Experimental results of the alpha test and heavy ion test were consistent with the results of the simulation. The cross sections of $6 \mathrm{~T}$ and 10T cells present much more significant sensitivities than Quatro and DICE cells for all tested supply voltages and LET. The 10T cell demonstrates a more optimal radiation performance than the 6T cell when LET is small $\left(0.44 \mathrm{MeV} \cdot \mathrm{cm}^{2} / \mathrm{mg}\right)$, yet no significant advantage is evident when LET is larger than this. In regards to the Quatro and DICE cells, one does not consistently show superior performance over the other in terms of soft error rates (SERs). Multi-bit upsets (MBUs) occupy a larger portion of total SEUs in DICE cell when relatively larger LET and smaller supply voltage are applied. It explains the
\end{abstract}


loss in radiation tolerance competition with Quatro cell when LET is bigger than 9.1 $\mathrm{MeV} \cdot \mathrm{cm}^{2} / \mathrm{mg}$ and supply voltage is smaller than $0.6 \mathrm{~V}$. In addition, the analysis of test results also demonstrated that the error amount distributions follow a Poisson distribution very well for each type of cell array. 


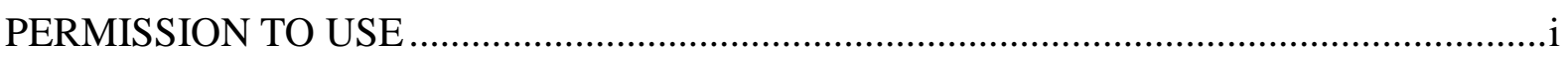

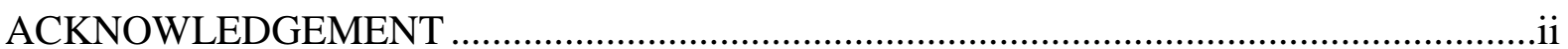

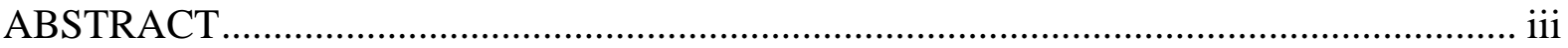

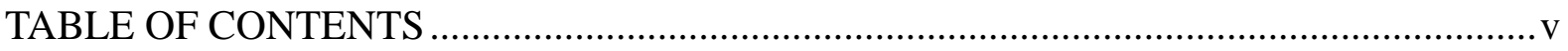

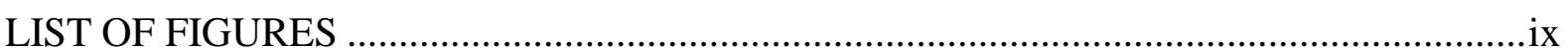

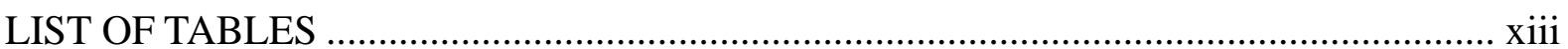

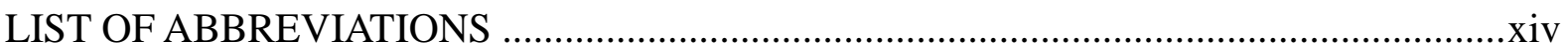

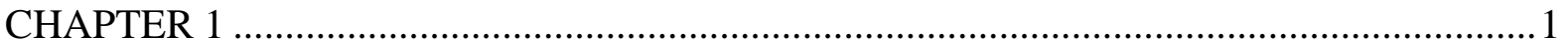

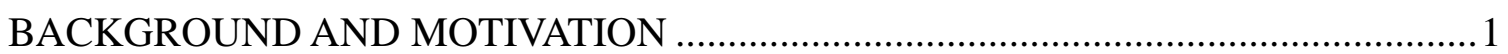

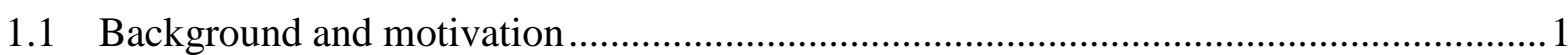

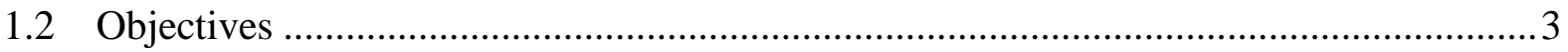

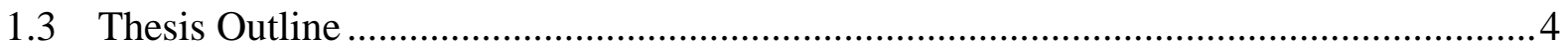

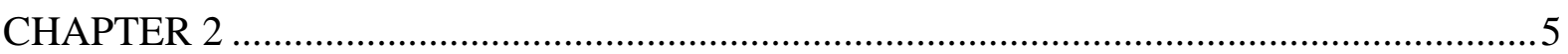

INTRODUCTION

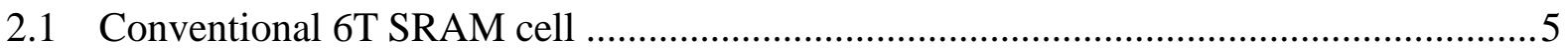

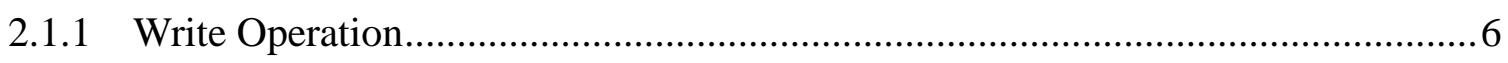

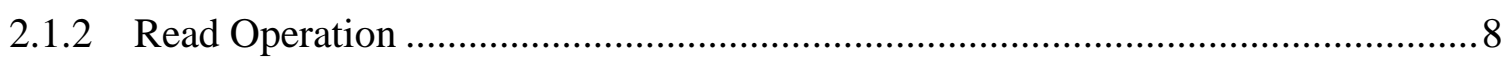

2.2 Challenges for Low Power Operation.................................................................. 10

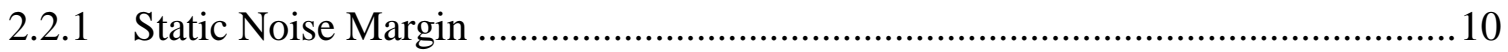

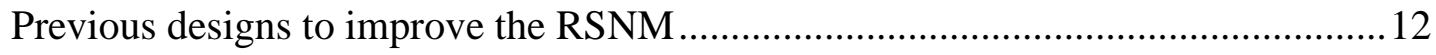

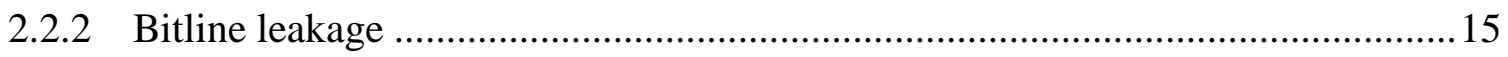


Previous design to reduce bitline leakage ........................................................ 17

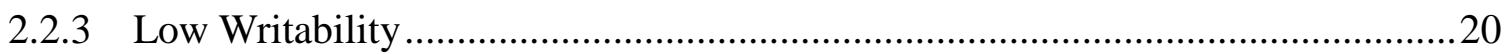

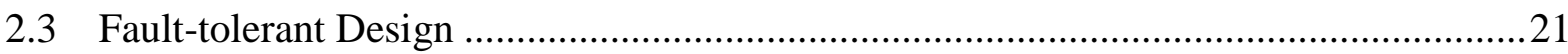

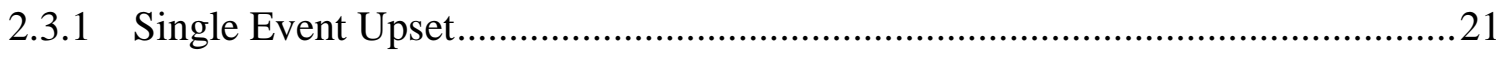

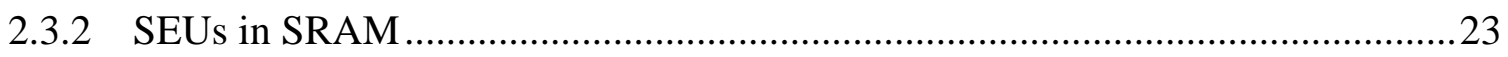

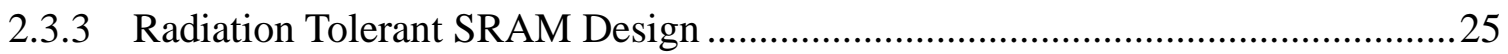

2.3.3.1 Dual Interlocked Storage Cell..........................................................25

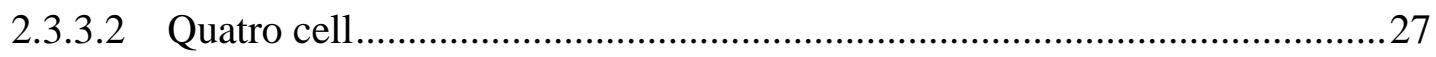

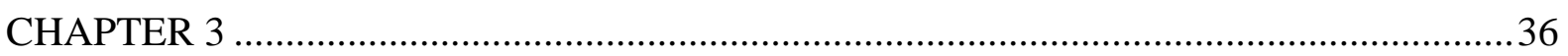

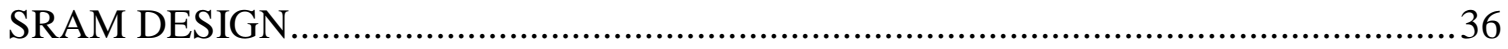

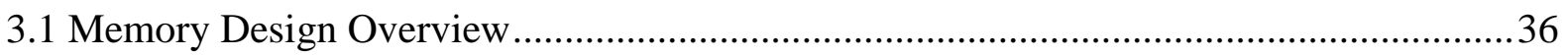

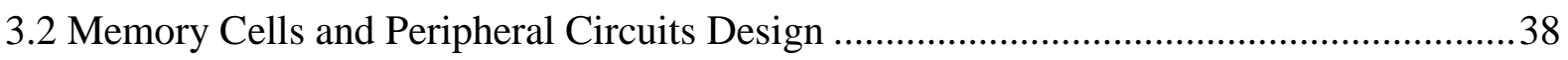

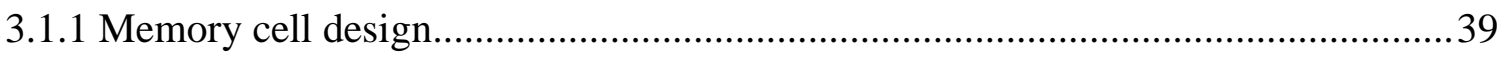

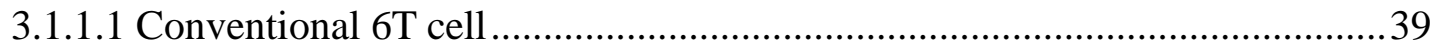

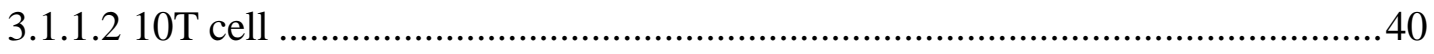

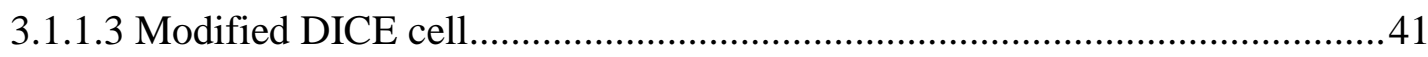

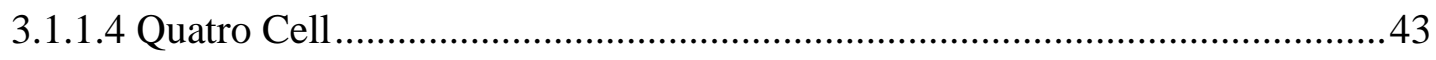

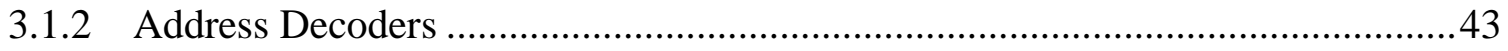

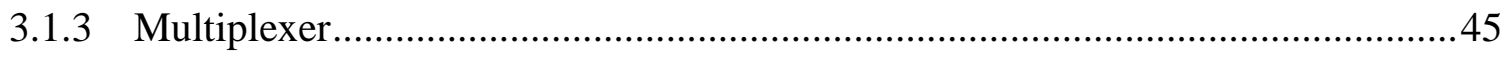

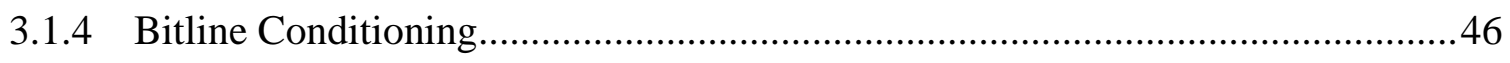

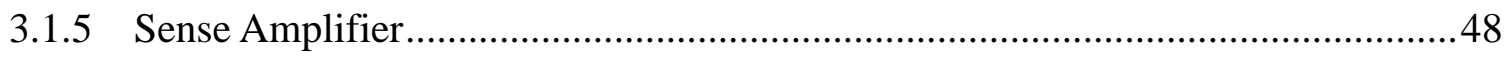

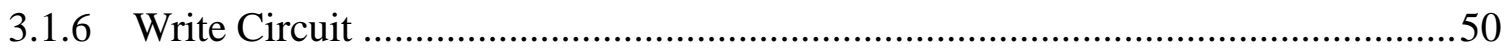




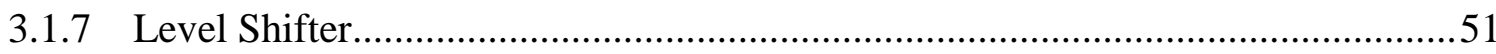

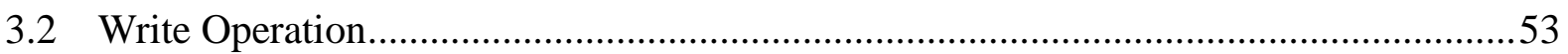

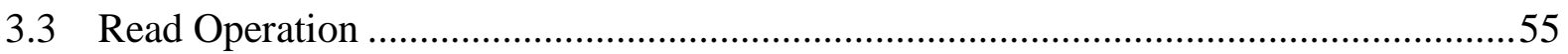

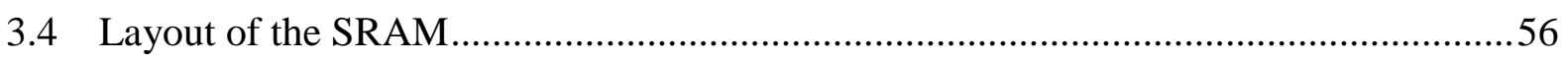

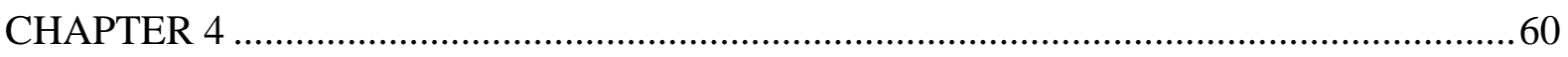

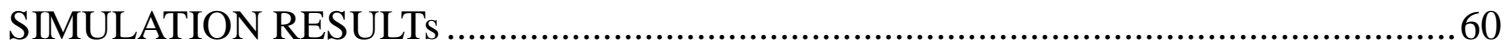

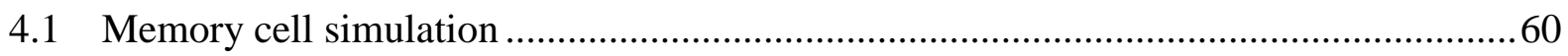

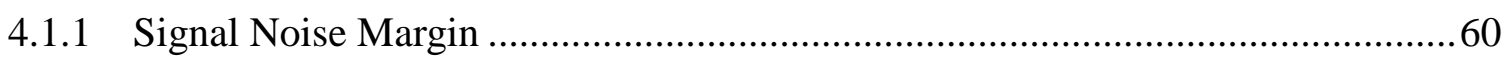

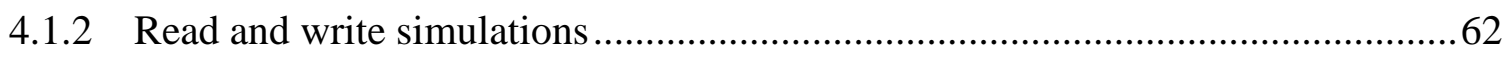

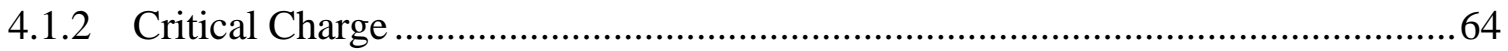

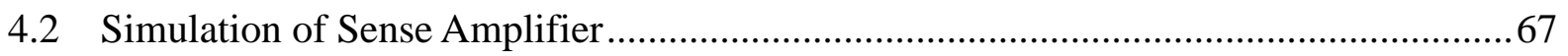

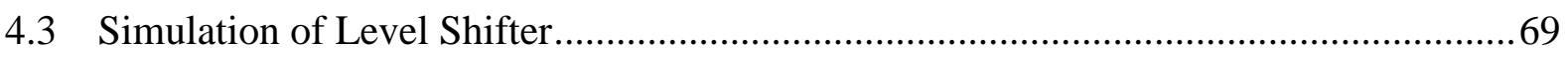

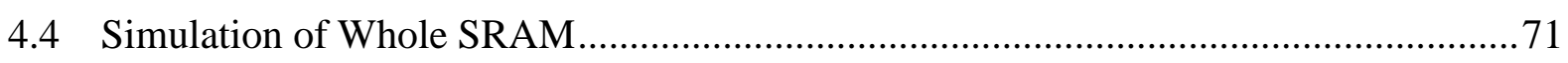

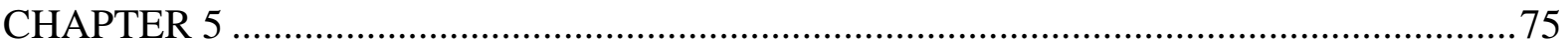

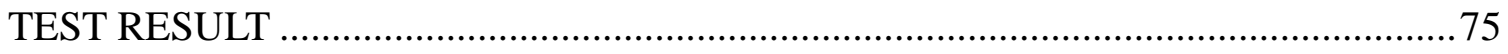

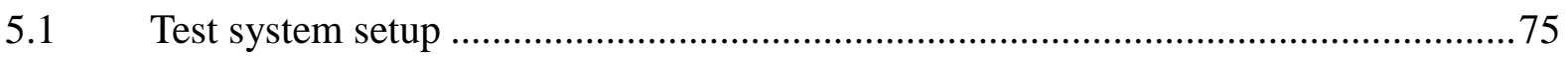

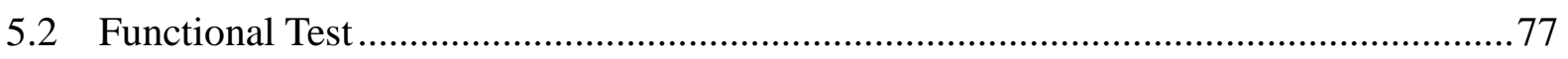

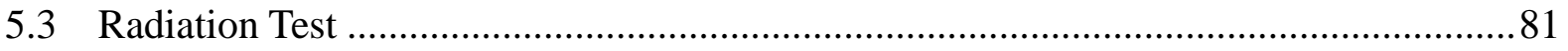

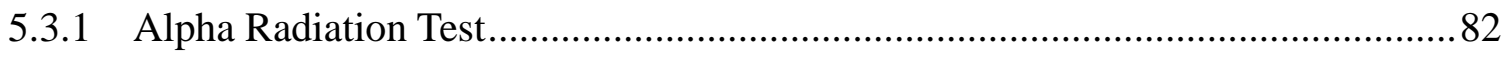

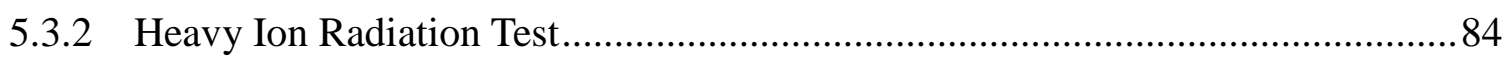

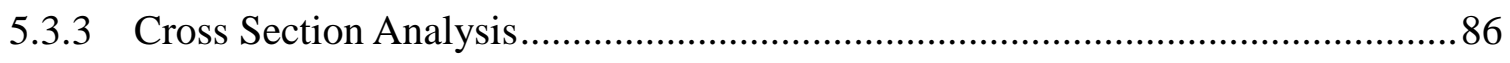

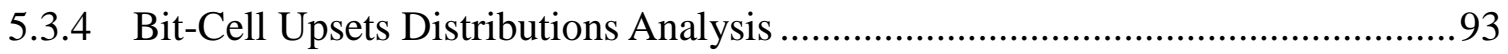




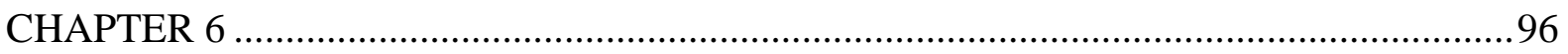

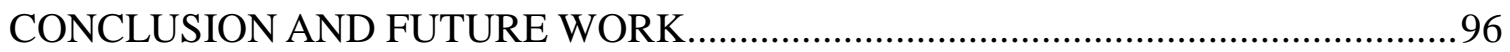

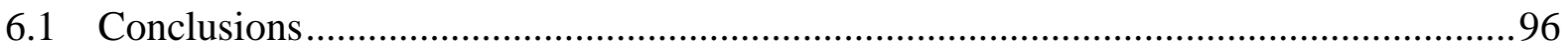

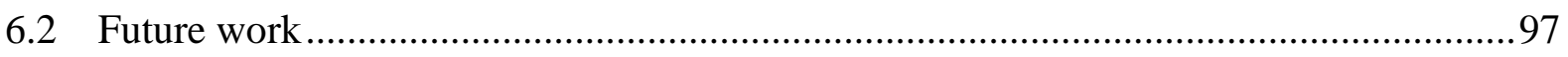

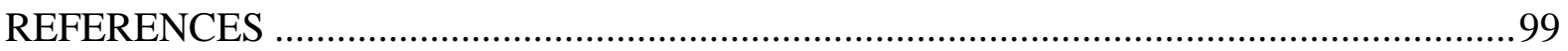




\section{LIST OF FIGURES}

Figure 2.1 Conventional 6T SRAM cell ...................................................................

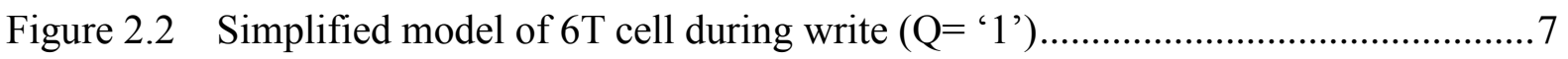

Figure 2.3 Write operation for 6T SRAM cell ..........................................................

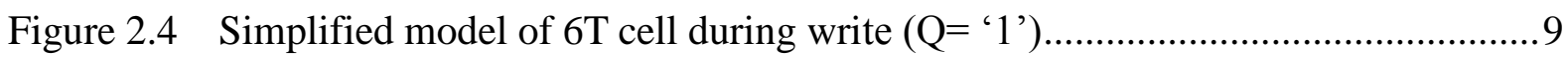

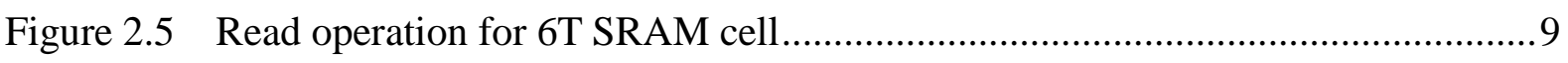

Figure 2.6 Cross-coupled inverters with noise source for HSNM simulation [24] ............. 10

Figure 2.7 Cross-coupled inverters with noise source for RSNM simulation [24] ............. 10

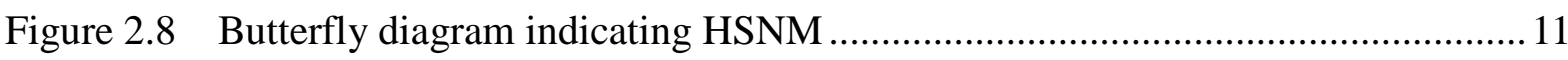

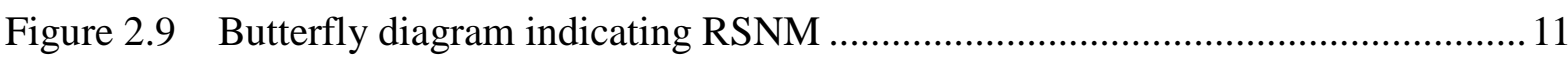

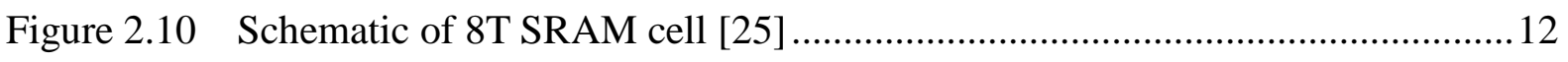

Figure 2.11 Schematic of Chang's 10T SRAM cell [13] ............................................... 14

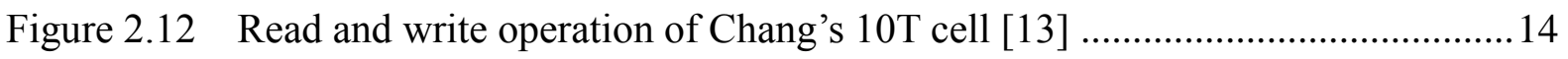

Figure 2.13 Worst case for bitline leakage [10] ......................................................... 15

Figure 2.14 Large data dependency induced by bitline leakage [11] ............................... 16

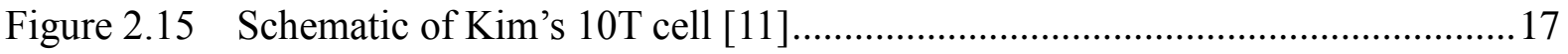

Figure 2.16 Improved small data dependency by bitline leakage [11] ............................. 18

Figure 2.17 Leakage with read buffer feet structure .................................................... 19

Figure 2.18 Boost circuit to drive read buffer [10] ..................................................... 20

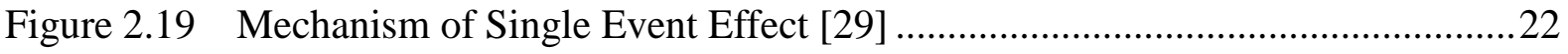

Figure 2.20 Single Event Effect current [29] ............................................................ 22

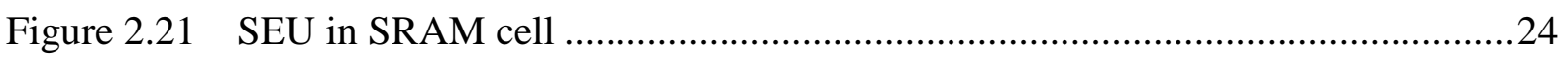




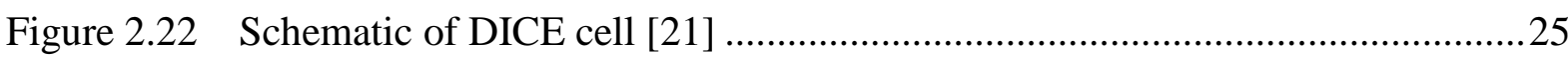

Figure 2.23 Simulation result of SEU effect on DICE cell...............................................26

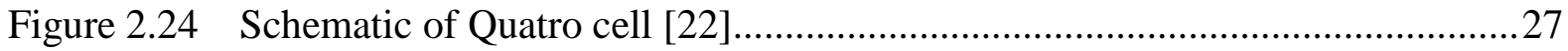

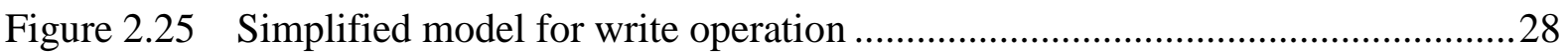

Figure 2.26 Simulation waveforms of the storage nodes, wordline, and bitlines in a write cycle .29

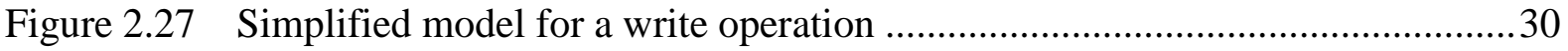

Figure 2.28 Simulation waveforms of the storage nodes, wordline, and bitlines in a write cycle 31

Figure 2.29 Recovery from injected current mimicking ' 1 ' to ' 0 ' at node A 32

Figure 2.30 Flipping of cell by injecting current mimicking ' 0 ' to ' 1 ' at node A 33

Figure 2.31 Recovery from injected current mimicking ' 0 ' to ' 1 ' at node $\mathrm{C}$ .34

Figure 2.32 Flipping of cell by injecting current mimicking ' 1 ' to ' 0 ' at node $\mathrm{C}$. .35

Figure 3.1 The block diagram for one page of the SRAM test chip .37

Figure 3.3 Schematic of $10 \mathrm{~T}$ cell 40

Figure 3.4 Schematic of low power operational DICE cell 41

Figure 3.5 Schematic of an Quatro cell .43

Figure 3.6 Pseudo read problem of hold cells .45

Figure 3.7 Global wordline and Local wordline [24] 45

Figure 3.8 Schematic of four to one multiplexer .46

Figure 3.9 Bitline conditioning circuit. .46

Figure 3.10 Controlled bitline conditioning circuit 47 
Figure 3.11 Timing sequence of $\Phi$ during read, write and hold operations .48

Figure 3.12 Schematic of latch based sense amplifier [40] 49

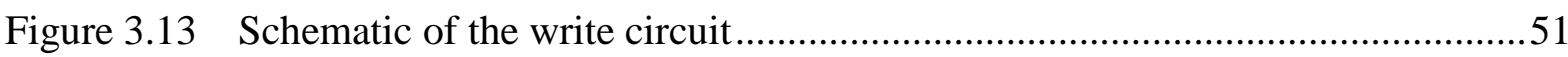

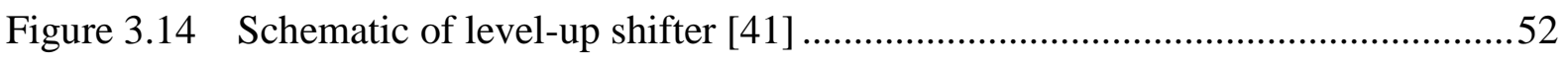

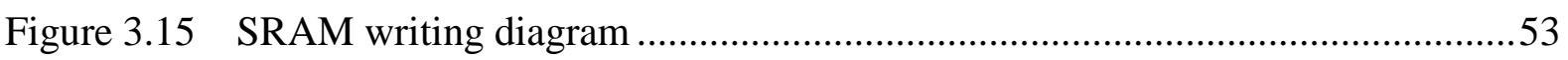

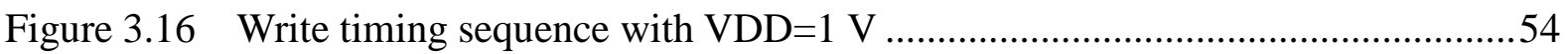

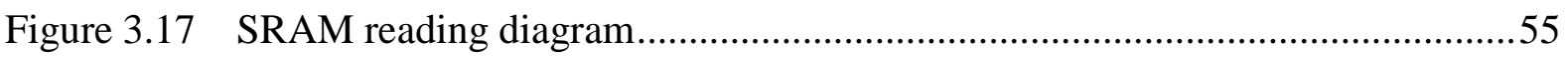

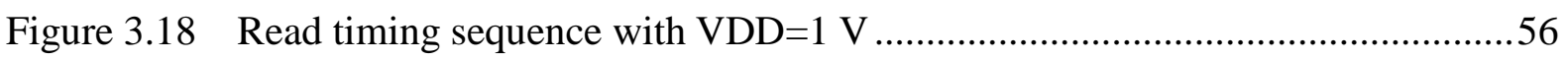

Figure 3.19 Layout of one quadrant of the SRAM design.............................................57

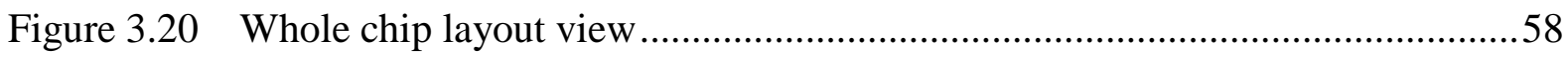

Figure 4.1 Read butterfly curves of the four SRAM cells ............................................... 61

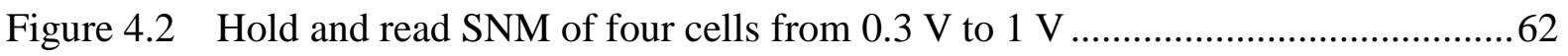

Figure 4.3 Simulation results of BL, BLB and RWL during read cycle with VDD=1 V and $0.3 \mathrm{~V}$ 63

Figure 4.4 Simulation results of storage nodes and RWL during read cycle with VDD=1 V and $0.3 \mathrm{~V}$ .63

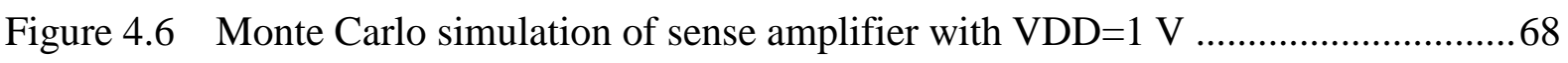

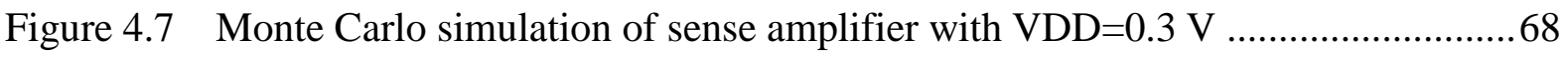

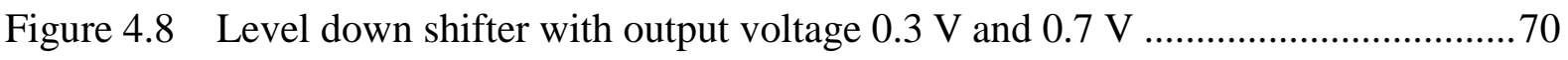

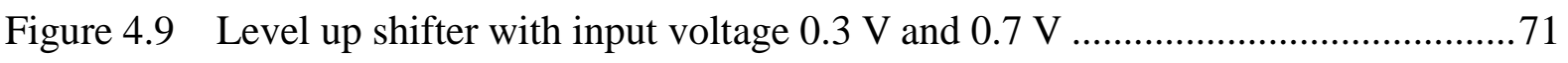

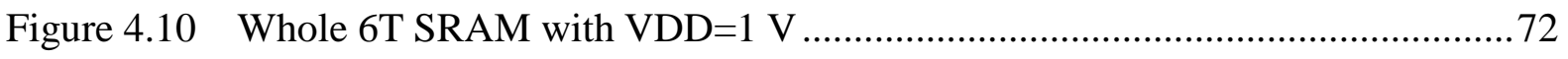

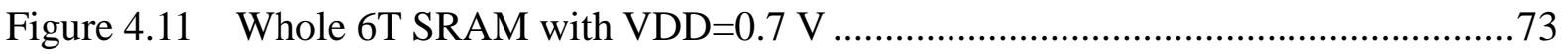




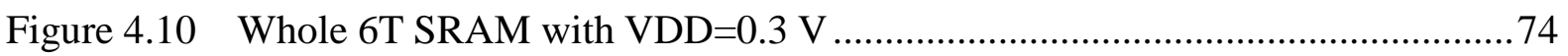

Figure 5.1 Photo of PCB with the SRAM chip soldered ................................................... 75

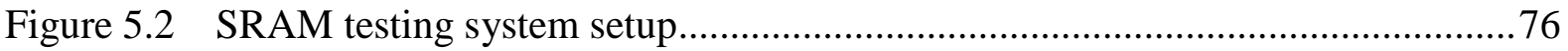

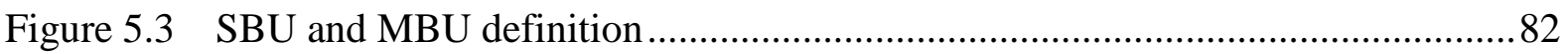

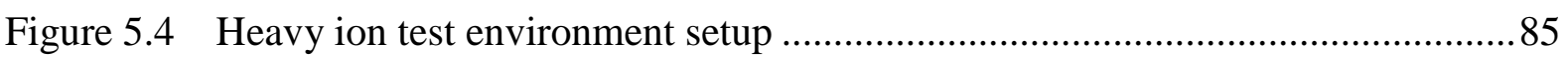

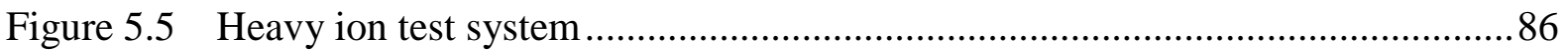

Figure 5.6 Cross section of the four SRAM cell arrays................................................ 90

Figure 5.8 Bit-cell upsets distributions of SRAMs .................................................... 94 


\section{LIST OF TABLES}

Table 3.1 Characteristics of SRAM Cells ........

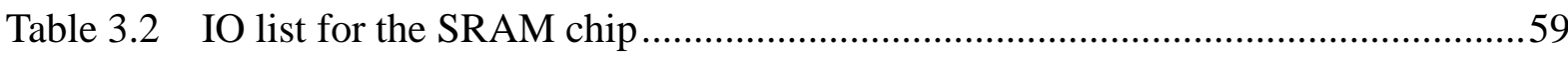

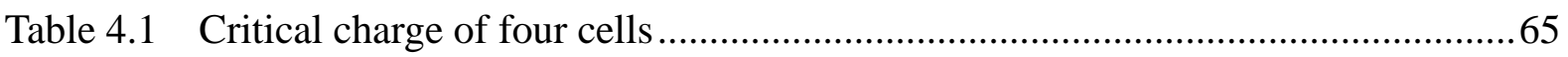

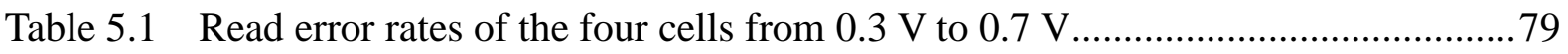

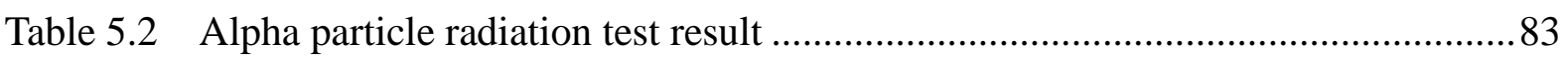

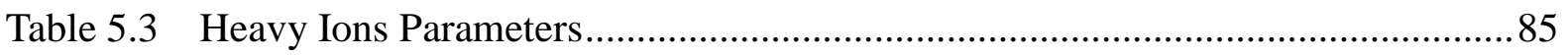

Table 5.4 SBU and MBU Cross Sections of the Four SRAM Cell Arrays ...........................93 


\section{LIST OF ABBREVIATIONS}

CIAE China Institute of Atomic Energy

CQFP80 Ceramic Quad Flat Package

CR Cell Ratio

DICE Drain-Induced Barrier Lowering

DIBL Dual Interlocked Storage Cell

DIMM Dual In-line Memory Module

FPGA Field-Programmable Gate Array

HSNM Hold Signal Noise Margin

LET Linear Energy Transfer

MBU Multi-bit Upset

MNU Multi-node Upset

MUX Multiplexer

PCB Printed Circuit Board

PR Pull-up Ratio

RHBD Radiation Hardened by Design

RSCE Reverse Short Channel Effect

SA Sense Amplifier

SBU Single-bit Upset

SEE Single Event Effect

SER Soft Error Rate

SEU Single Event Upset 
SNM

SRAM

SSSC

WSNM

VTC
Signal Noise Margin

Static Random Access Memory

Saskatchewan Structural Sciences Centre

Write Signal Noise Margin

Voltage Transfer Characteristic 


\section{CHAPTER 1 \\ BACKGROUND AND MOTIVATION}

\subsection{Background and motivation}

SRAM (Static Random Access Memory) occupies a large area of most modern nanometer chips and consumes a large portion of power consumption [1]. With advanced processes scaling down, power consumption has already become an important factor for large-scale SRAMs design. SRAM operating in lower power supply voltages has become an effective approach to reduce power consumptions. However, radiation immunity for memory is also critical, considering the fact that the error caused by Single Event Upset (SEU) can be "remembered" by the SRAM, ultimately resulting in a vital functionality fault. In addition, lowering the supply voltage may also lead to the reduction of nodal critical charge, thus imposing acute soft error threats due to single events on the reliable operations of SRAMs [10]. Therefore, the estimations of SRAM Soft Error Rates (SER) versus voltage relationships are critical in determining their appropriate applications.

In order to study the supply power dependence of radiation effects on SRAM, especially in sub-threshold region, two factors must be studied: low power operational and SEU tolerance.

With the increasing applications in space, biomedical, mobile and other battery based devices, reducing the power dissipation has become an important objective in chip design. The total power consumption in the chip can be divided to two portions: dynamic dissipation and static dissipation, as shown in the equation below [2]. 


$$
\begin{aligned}
& P_{\text {total }}=P_{\text {static }}+P_{d y n a m} \\
& P_{\text {static }}=I_{\text {static }} V_{D D} \\
& P_{d y n \text { amic }}=\alpha C V_{D D}^{2} f
\end{aligned}
$$

In reference to the outlined equation, $f$ represents clock frequency and $\alpha$ is referred to as activity factor, which is used to describe the switching frequency of one gate as $\alpha$ times the clock frequency. $C$ is the equivalent capacitance between $V_{D D}$ and ground. Considering the fact that both dynamic power and static power are increased with $V_{D D}$, reducing the supply voltage is an efficient way to reduce the power consumption when speed is not the predominant consideration.

However, challenges begin to emerge in cases where the supply voltage $V_{D D}$ decreases to the sub-threshold region. The main challenges include the low Static Noise Margin (SNM) [3], bitline leakage, and writability [11]. In this case, significant efforts have been made to the study of overcoming the challenges for developing sub-threshold SRAMs [4], [5], [6].

In regards to SRAM fabricated in nanometer technologies, scaled size results in heightened vulnerability to single event effects. Furthermore, decreased supply power also decreases the robustness of memory cells to SEUs, considering the fact that the energy required to flip a cell is significantly reduced [7], [8], [9]. In the devices that require high reliability, such as mainframes or space, the radiation effects must be taken into consideration. The majority of sub-threshold region SRAM cells employ inverter-loops, which are sensitive to single event effects; therefore, these sub-threshold SRAM structures are more vulnerable to SEUs [10]-[15]. Among the radiation hardness by design (RHBD) 
cells, the dual-interlocked storage cell (DICE) may be considered the most well-known and widely used of these cells [16]. In 2009, another RHBD SRAM cell named Quatro was proposed [17], which is a cell that uses fewer transistors as compared to DICE. Correspondingly, radiation experiments (neutron, alpha, and heavy ions) demonstrated a higher radiation tolerance than DICE in cases where both of the cells were used to construct flip-flops in a 40nm technology [18].

In previous literature, the supply voltage dependences of alpha and neutron radiation effects in unhardened SRAM cells have been studied in [19]-[21]. For a SRAM fabricated with 90nm CMOS process, when supply voltage decreases by every $10 \%$, the measured SER induced by neutron increases by $18 \%$ [19]. It is also reported that the multi-bit upsets (MBU) rate of a $65 \mathrm{~nm}$ 10T SRAM caused by alpha and neutron sources increase as the power supply voltage decreases to sub-threshold [20] [21]. However, as far as the author knows, supply power dependences on unhardened and hardened SRAMs when irradiated with heavy ions have not been reported yet. The advantage of using heavy ions is that it is able to provide a wider range of linear energy transfers (LETs) than alpha particles, as well as facilitating a more accurate mechanism of the interactions with silicon [22]. Therefore, heavy ion experiments on SRAMs can directly reveal the LET impact on the SER/voltage relationship.

\subsection{Objectives}

The objectives of this research are as follows:

1. The comprehensive study of supply power dependences of the single-event effects (SEEs) of four different SRAM cells. Design and fabricate a SRAM with different cells, some are normal cells and some are modified for low power operational. These 
cells include both hardened and unhardened cells, irradiated by heavy ions with variable energy. Upon completion of the study, the results must be compared with an alpha test and simulation results.

2. The investigation of bits-cell upset distribution and the analysis of single-bit upset and multi-bits upset on four types of memory cells.

\subsection{Thesis Outline}

The remainder of the thesis is organized as follows: In chapter 2, some challenges in sub-threshold region SRAM design and previous studies for solving the challenges are introduced. Additionally, the mechanism of SEE and SEU in SRAM and two radiation hardened SRAM cell, including DICE Cell and Quatro cell, are also introduced. In chapter 3, the whole SRAM structure, four types of SRAM cells, each part of peripheral circuits and the read and write operations are introduced in detail. Chapter 4 outlines the simulation results of the SRAM, while chapter 5 summarizes the testing results from functional, alpha particles and heavy ion experiments. In addition, the testing results are analyzed and discussed in chapter 5. Chapter 6 concludes the work in this thesis and investigates future research objectives. 


\section{CHAPTER 2 \\ INTRODUCTION}

In order to study the voltage dependence of radiation effects on SRAM cells, the operation principle of low power SRAMs as well as the Single Event Effects (SEEs) on SRAMs must be studied. The first part of this chapter introduces the commonly used $6 \mathrm{~T}$ SRAM cell and the principle of its read and write operations. Following this introduction, the challenges for subthreshold operation of SRAMs are listed and some previous designs to overcome each challenge are presented in the second part of this chapter. Finally, the general mechanism of SEEs in SRAM cells and some previous designs for radiation-hardened designs are introduced.

\subsection{Conventional 6T SRAM cell}

The most conventional and commonly used SRAM cell is the standard 6-transistor SRAM [23], as in Figure 2.1.

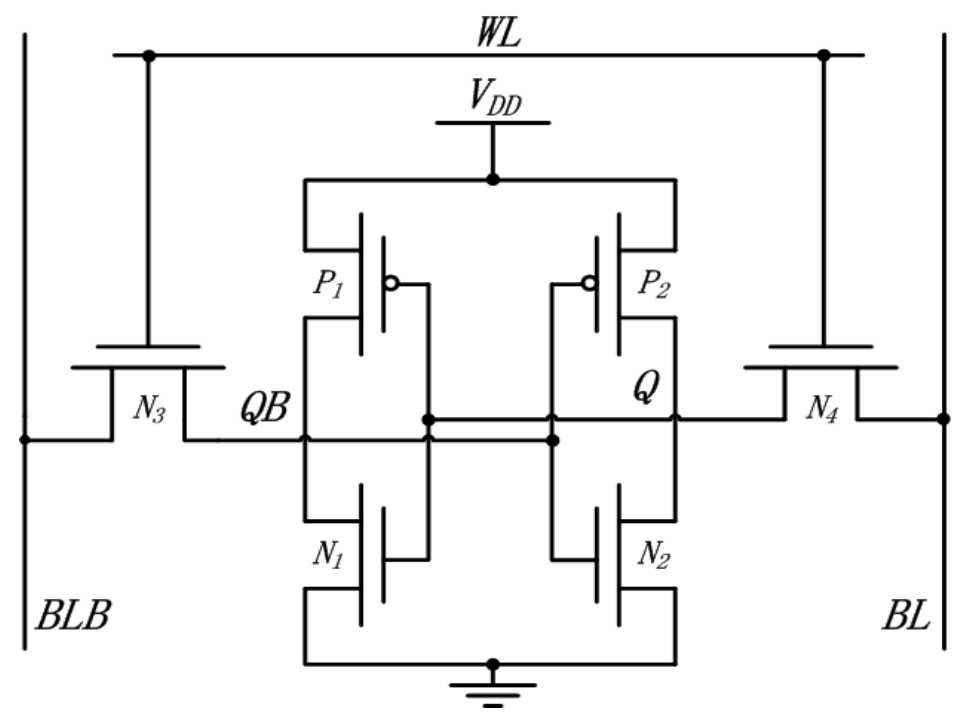

Figure 2.1 Conventional 6T SRAM cell 
The 6T cell contains a pair of cross-coupled inverters $(P 1, N 1$ and $P 2, N 2)$ to secure the states and a pair of access transistors (N3 and N4) driven by wordline (WL) and connected to bitlines $(B L$ and $B L B)$ to read or write the state from the nodes $Q$ and $Q B$. The positive feedback in the cross-coupled inverters corrects the disturbance of noise and leakage to maintain the states. In order to ensure the functionality of the 6T SRAM cell, some transistor-sizing constraints must be fulfilled.

\subsubsection{Write Operation}

To study the write operation of 6T cell, a simplified model of the 6T SRAM cell during write operation is shown as Figure 2.2. It can be assumed that the initial state of the cell is $Q={ }^{\prime} 1$ ', and we wish to write ' 0 ' to $Q$. $B L B$ is driven to high and $B L$ is pulled down to low by the input driver. $W L$ is asserted, turning both $N 3$ and $N 4$ on to open access to node $Q$ and $Q B$. Note that $Q$ must be pulled low enough to ensure reliable writing to the cell, that is, below the threshold voltage $V_{t n}$ of $N 3$. Once $Q$ falls below $V_{t n}, P 1$ is turned on and $N 1$ is turned off, pulling $Q B$ high as desired. In order to satisfy this condition, the drivability of $N 4$ must be bigger than $P 2$. The pull-up ratio of the cell, $P R$, which is defined as the size ratio between the PMOS and the access NMOS (naming $P 2$ and N4), $P R=\frac{W_{P 2} / L_{P 2}}{W_{N 4} / L_{N 4}}$ must be smaller than 1.8 [23]. Figure 2.3 illustrates the waveforms for the write operation. In correspondence with the assumption above, ' 0 ' is written into $Q$, which was initially ' 1 '. 


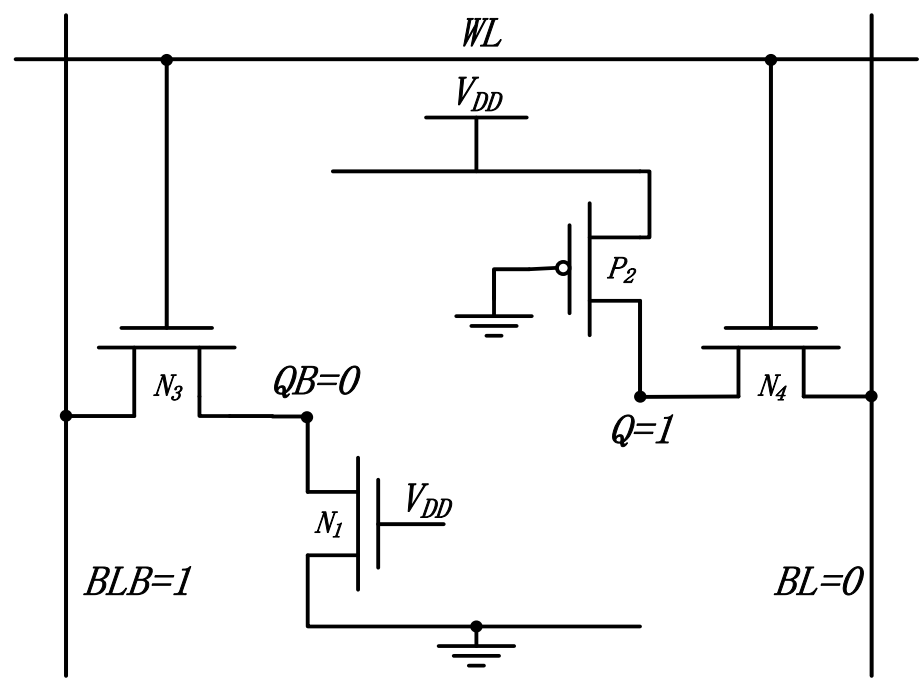

Figure 2.2 Simplified model of $6 \mathrm{~T}$ cell during write $\left(Q={ }^{\prime} 1\right.$ ')

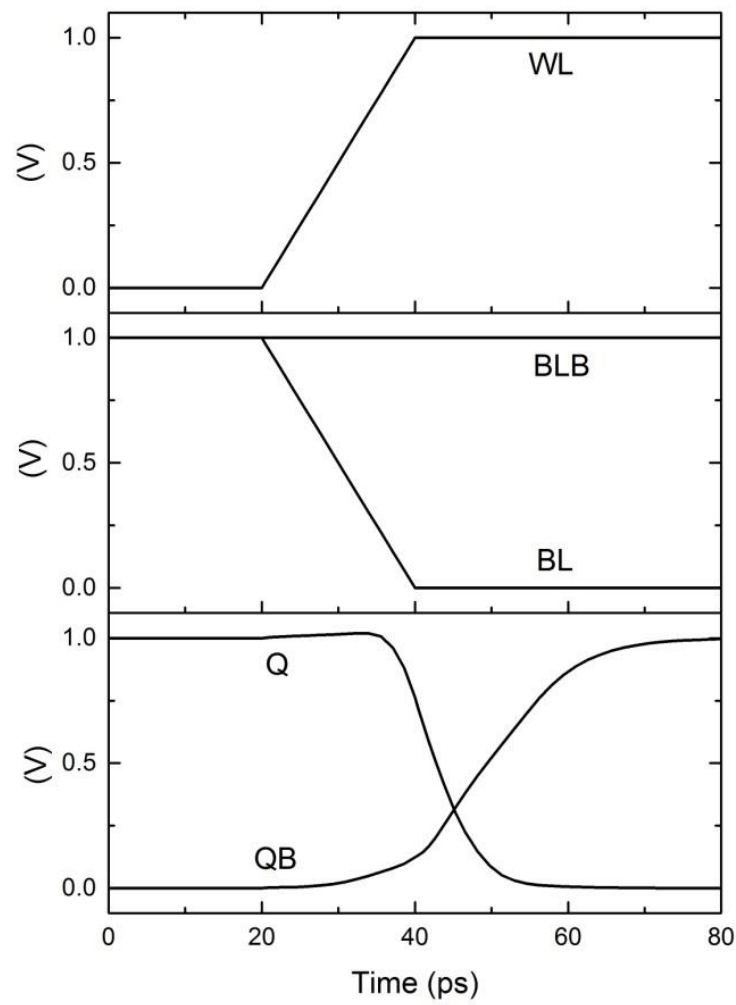

Figure 2.3 Write operation for 6T SRAM cell 


\subsubsection{Read Operation}

In order to understand the read operation, assume that ' 1 ' is stored at $Q$ again. Similar to the write operation, a simplified model of the 6T SRAM cell during read operation is also shown in Figure 2.4. Before the reading operation, both bitlines $B L$ and $B L B$ are initially precharged to high. The read operation starts by asserting $W L$ high and enabling the access transistor N3 and N4. During the read cycle, ' 0 ' stored in $Q B$ pulls down $B L B$ towards to ground. As many cells are connected to one bitline in SRAM, there is a large capacitance $C_{b i t}$ existing between bitline and ground, which decelerates the pulling down process. When the difference between $B L$ and $B L B$ begins to build up, sense amplifier will be enabled to amplify the difference and accelerate the reading process.

At the rising time of $W L$, the initial precharged high voltage tends to disturb $Q B$ and pull it high, and the cell can subsequently be flipped if this shifting on $Q B$ is too large. The voltage rise on $Q B$ must be kept low to maintain high reading reliability. This stipulates that $N 3$ must be weaker than $N 1$. Another constraint cell ratio $C R=\frac{W_{N 1} / L_{N 1}}{W_{N 3} / L_{N 3}}$ must be fulfilled for read stability, that is, CR must be greater than 1.2 [23]. Figure 2.5 outlines the waveforms for $Q={ }^{\prime} 1$ ' as read from the SRAM cell. 


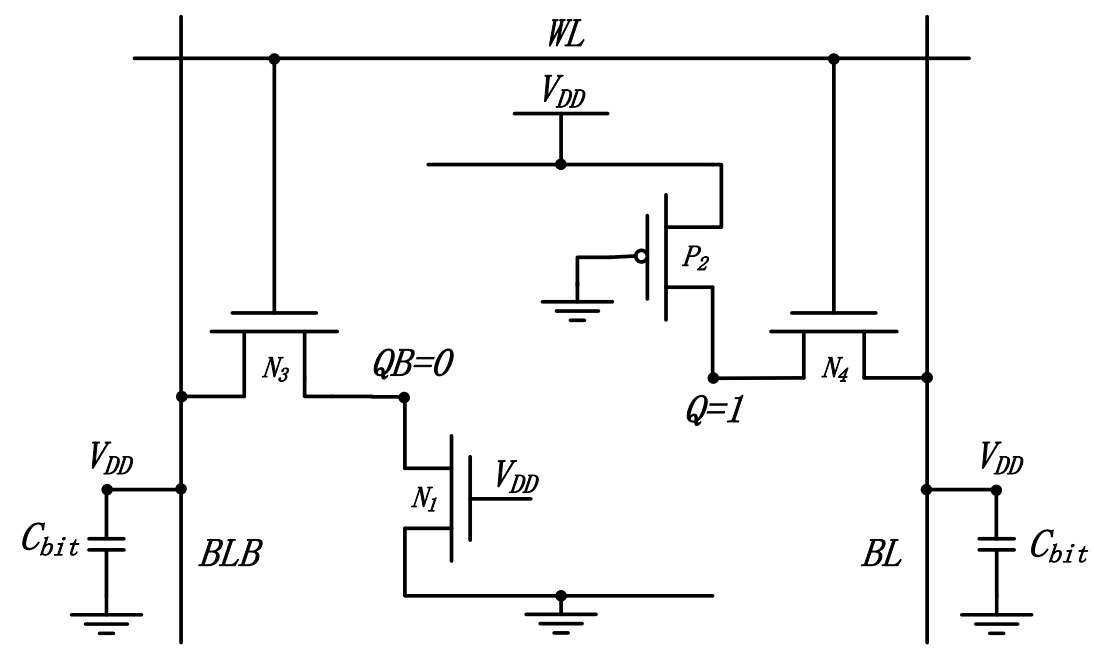

Figure 2.4 Simplified model of $6 \mathrm{~T}$ cell during write $(\mathrm{Q}=$ ' 1 ')

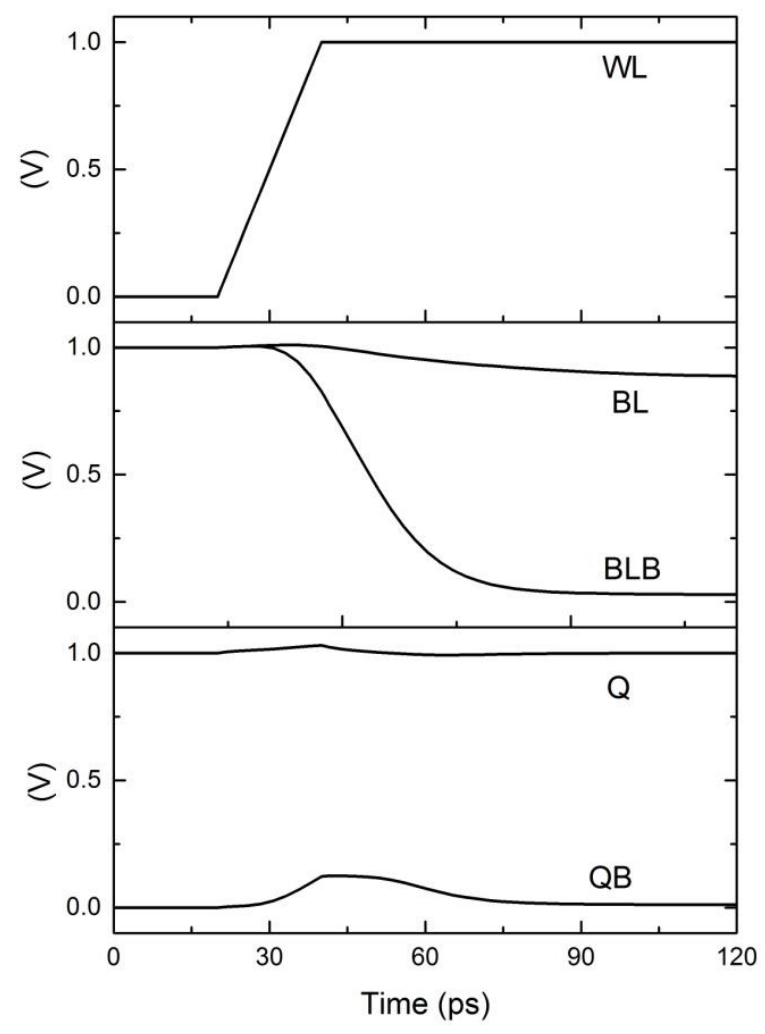

Figure 2.5 Read operation for 6T SRAM cell

In order to ensure both readability and writability, the pull-up ratio $P R$ and cell ratio $C R$ must be satisfied simultaneously. According to the constraints $P R$ and $C R$, pull-down 
nmos N1 and N2 must be the strongest. Access transistors N3 and N4 should have medium strength, and the pull-up PMOS must be the weakest. Meanwhile, all transistors must be as small as possible in order to achieve higher layout density.

\subsection{Challenges for Low Power Operation}

\subsubsection{Static Noise Margin}

The stability of SRAM cell is quantified by Signal Noise Margin (SNM), which can be further divided to Hold Signal Noise Margin (HSNM), Read Signal Noise Margin (RSNM) and Write Signal Noise Margin (WSNM). SNM is defined as the maximum noise that can be applied to the cross-coupled inverters before the stable state is disturbed. Figure 2.6 and 2.7 illustrate the test circuit to measure the Hold Signal Noise Margin and Read Signal Noise Margin, respectively.

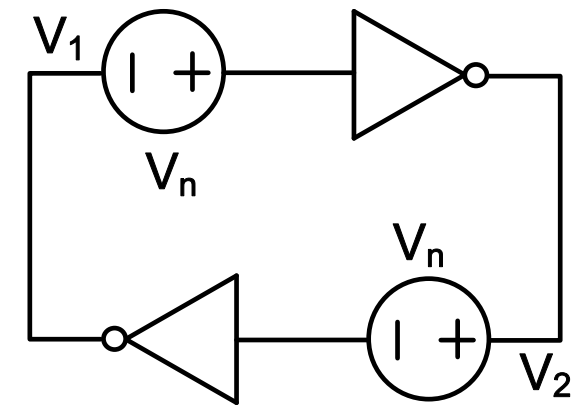

Figure 2.6 Cross-coupled inverters with noise source for HSNM simulation [24]

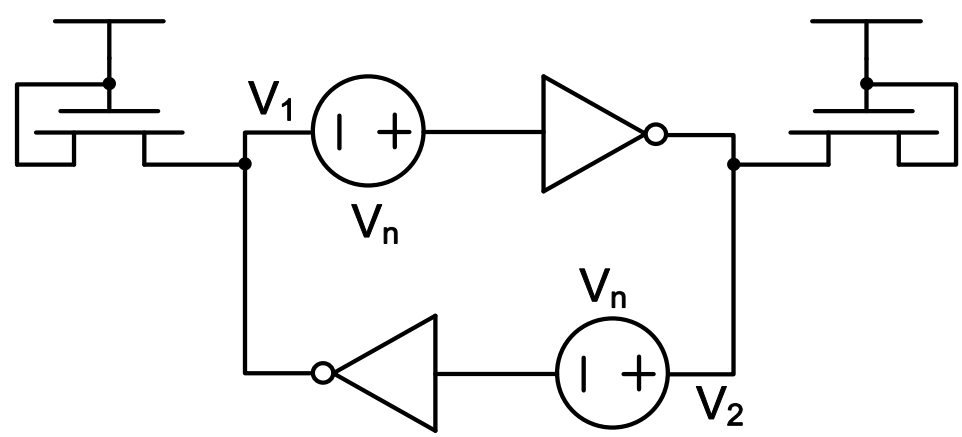

Figure 2.7 Cross-coupled inverters with noise source for RSNM simulation [24] 


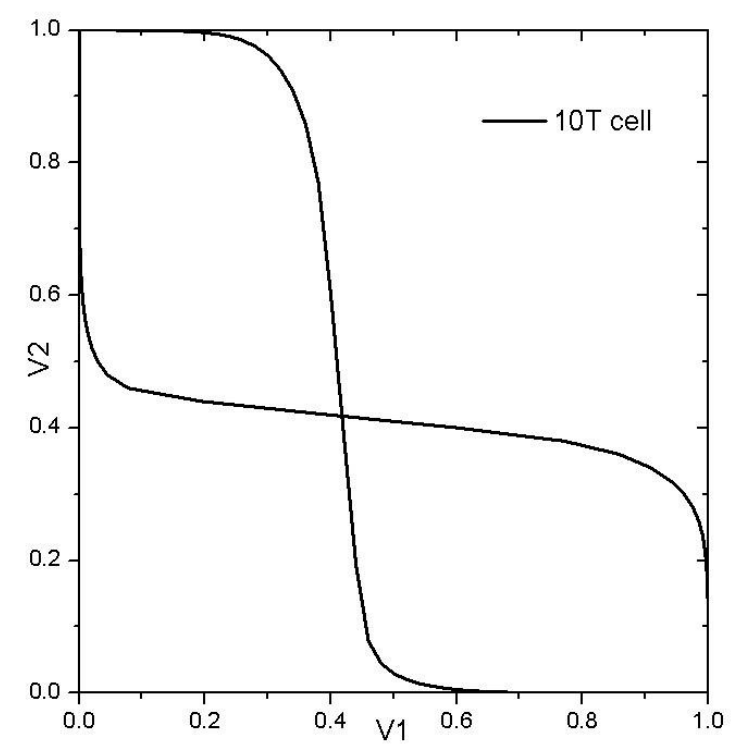

Figure 2.8 Butterfly diagram indicating HSNM

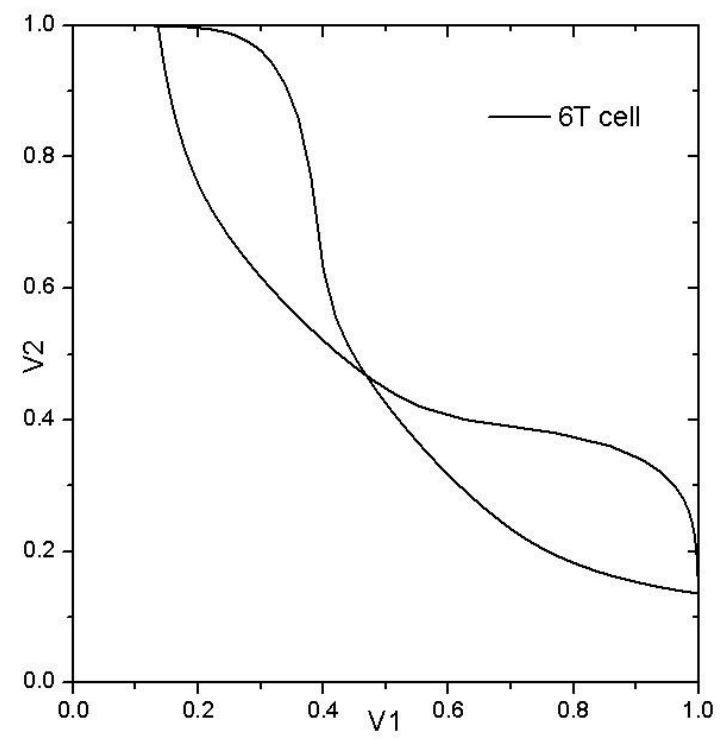

Figure 2.9 Butterfly diagram indicating RSNM

SNM can be determined from the butterfly diagrams as shown in Figure 2.8 and 2.9.

The butterfly curves are plotted by setting $V_{n}=0$, plotting $V_{1}$ against $V_{2}$ and $V_{2}$ against $V_{l}$, respectively. SNM can be obtained by the size of maximum square inside the two cross-coupled curves. As it can be seen, RSNM is significantly smaller than HSNM, as the cross-coupled inverters are affected by the open access to $V_{D D}$. RSNM is determined by the 
cell ratio- $C R$; a higher $C R$ increases the read margin in the trade off of taking more area for the pull-down transistors $N 1$ and $N 2$. In this thesis, when we come to the term $S N M$, it is referred to as $R S N M$.

\section{Previous designs to improve the RSNM}

\section{Chang's 8T cell}

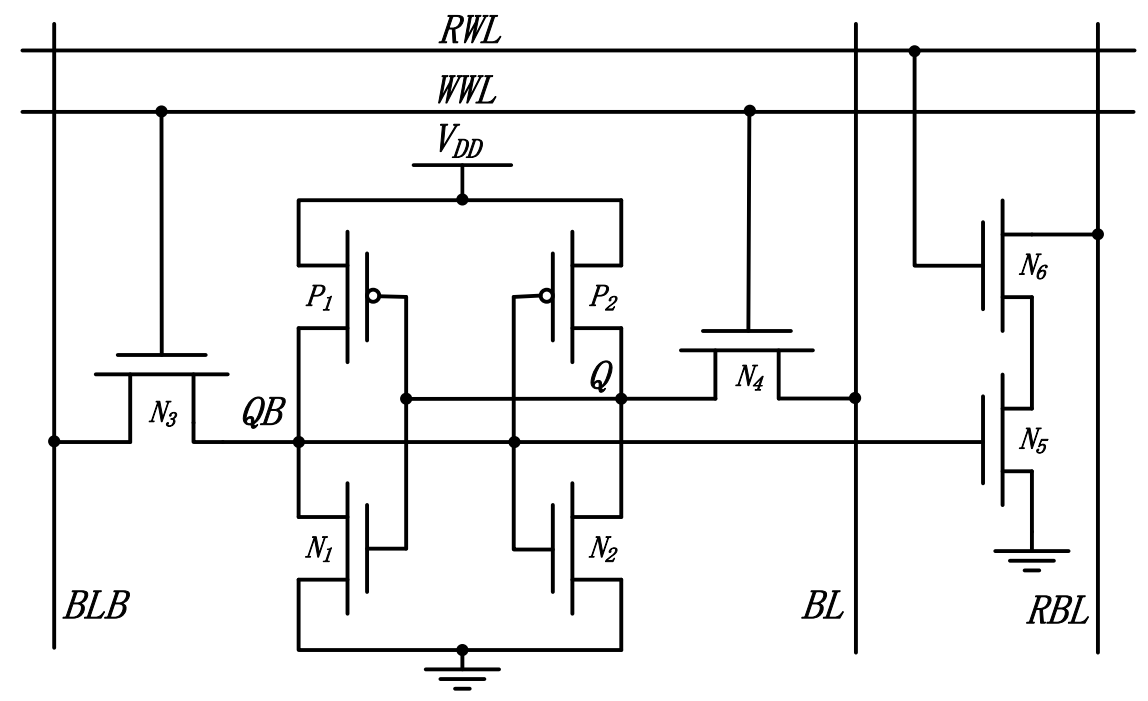

Figure 2.10 Schematic of 8T SRAM cell [25]

An 8T SRAM cell providing separate reading and writing operation was proposed by Chang [25] as in Figure 2.10. Two more transistors, N5 and N6, and one read wordline ( $R W L)$ are added to 6T SRAM cell to improve the stability of the read operation. During the write operation, $R W L$ is turned low to ensure that $N 6$ is off, blocking the path from group in order to read bitline $R W L$. $W W L$, in turn, is set to high. Therefore, in terms of the write operation, there is no difference between Chang's 8T cell and the conventional 6T cell. For a read cycle, assume data stored in the cell is ' 0 ', that is $Q={ }^{\prime} 0$ ' and $Q B={ }^{\prime} 1$ '. $R W L$ is asserted to turn on $N 6$, while $W W L$ keeps ' 0 ' to turn off $N 3$ and $N 4$. $N 5$ is also on as $Q B=$ ' 1 '. In this case, an open path from ground to $R B L$ is constructed to pull $R B L$ down. If cell content is ' 1 ', that is 
$Q=$ ' 1 ', $Q B=$ ' 0 ', $R B L$ will remain high as $N 5$ is turned off. It should be noted that $Q B$ is connected to the gate of $N 5$ rather than the diffusion area. Therefore, the initial high voltage on $R B L$ will not disturb the cell state. In this respect, the $S R N M$ has the same value as the $H S N M$

According the test result, the $8 \mathrm{~T}$ cell SRAM functions at frequency of $295 \mathrm{MHz}$ with $V_{D D}=0.41 \mathrm{~V}$, and with a dramatic leakage current deduction of over $60 \mathrm{x}$ compared to $1.2 \mathrm{~V}$ power supply operation. One drawback of Chang's $8 \mathrm{~T}$ cell is that it cannot take advantage of a small-signal sensing amplifier, which is able to distinguish small differences between $B L$ and $B L B$, as it has only one read bitline. Another disadvantage is that it does not allow physically interleaving bits from different words. Therefore, an additional parity or ECC bits are required to prevent multi-bit errors.

\section{Chang's 10T SRAM cell}

To solve the non-interleaving drawback of Chang's 8T cell, 10T SRAM cell was proposed by Chang [13]. The 10T cell provides isolated read and write operation as well as enables interleaving bits. As illustrated in Figure 2.11, four n-transistors (N5 N8) and another wordline $W_{-} W L$ is added on the basis of the conventional 6T cell. The source of $N 5$ and N6 are connected to $V G N D$ instead of $G N D$. The timing sequence for $V G N D, W_{-} W L$ and $W L$ is outlined in Figure 2.12. 


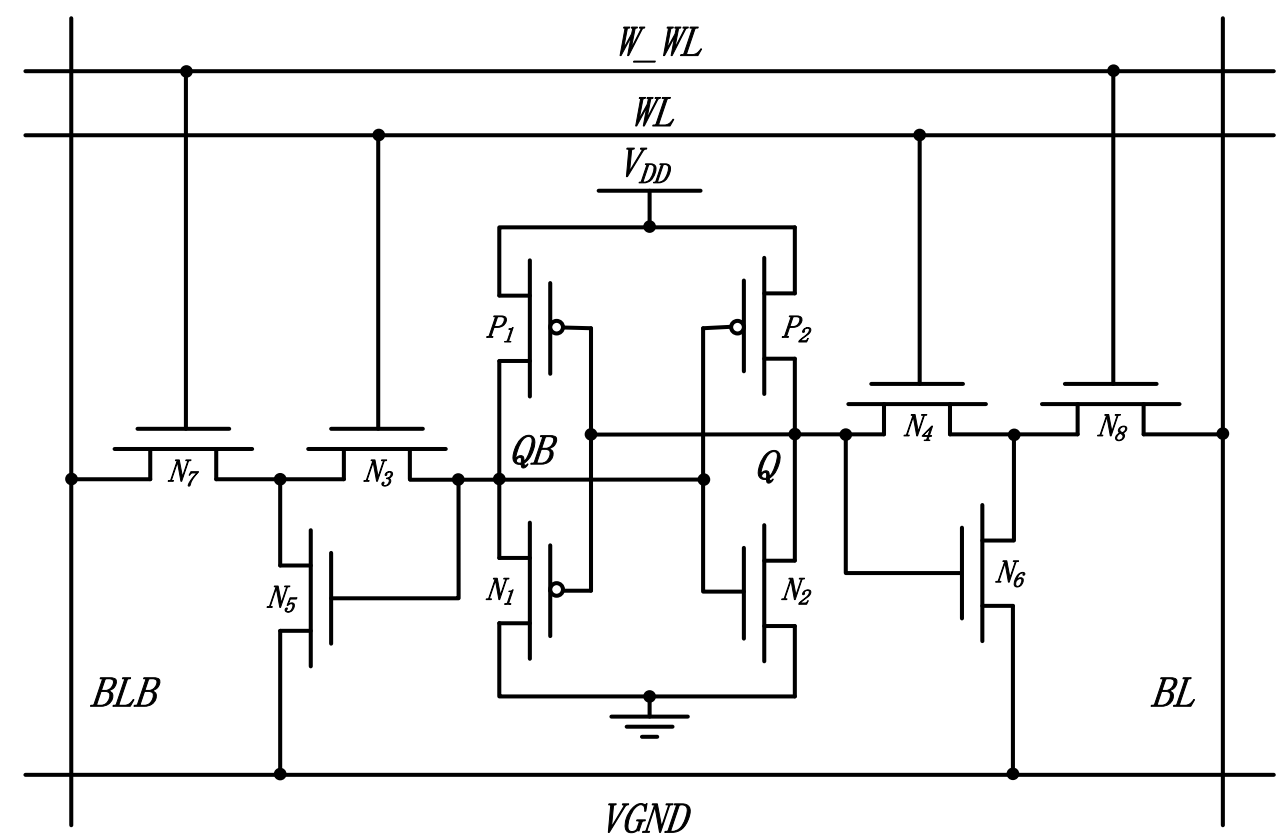

Figure 2.11 Schematic of Chang's 10T SRAM cell [13]

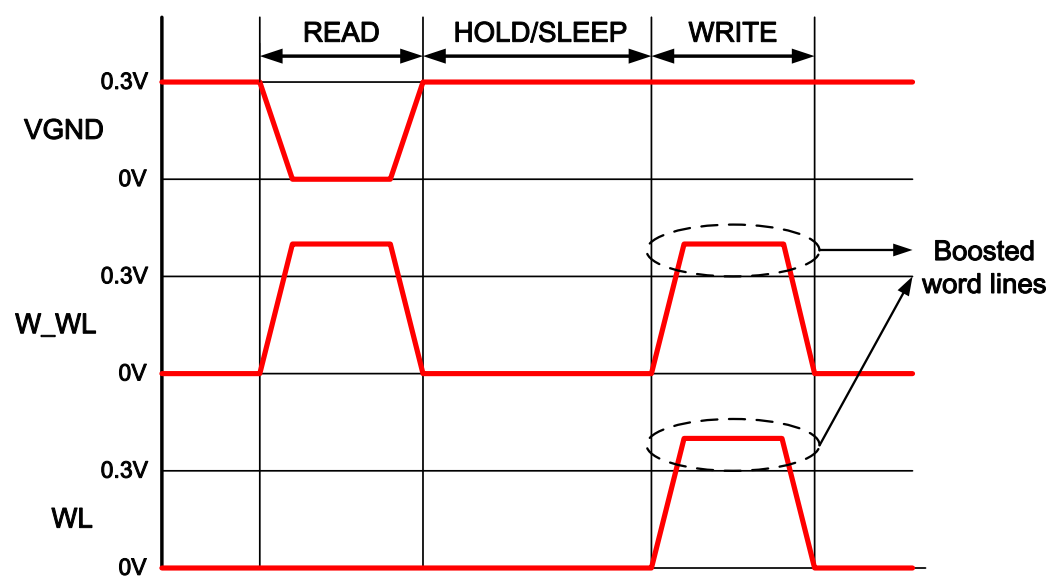

Figure 2.12 Read and write operation of Chang's 10T cell [13]

For read operation, again, assume data stored in the cell is ' 0 ', that is $Q=$ ' 0 ' and $Q B=$ ' 1 '. During a read operation, $W_{-} W L$ is high, while $W L$ and $V G N D$ are set to be low. In this case, $N 7$ and $N 8$ are on, while $N 3$ and $N 4$ are off, and N5 is on and N6 is off. $B L$ maintains precharged high voltage, as accesses from memory cell to $B L(N 8, N 4$ and $N 8, N 6)$ are closed. On the other side, an open path $(N 5, N 7)$ from $V G N D$ to $B L B$ is constructed to pull $B L B$ low. 
As shown in the schematic, there is no open access from the precharged $B L$ or $B L B$ to $Q$ or $Q B$ that could potentially disturb the node. Due to this isolation, the read signal noise margin is almost as big as the hold noise margin of the $6 \mathrm{~T}$ cell.

During the write operation, both $W L$ and $W_{-} W L$ are asserted to transfer data from binlines to $Q$ and $Q B$. However, because of the series access transistors and high potential on $V G N D$, the writability of Chang's $10 \mathrm{~T}$ cell becomes a critical issue especially in subthreshold application. To overcome this weakness, Chang also proposed to boost the $W L$ and $W_{-} W L$ by $100 \mathrm{mV}$ (at $300 \mathrm{mV} V_{D D}$ ) to compensate for the weak writability. Test result indicate that Chang's 10T SRAM demonstrates functionality at160 $\mathrm{mV} V_{D D}$ for read and $180 \mathrm{mV} V_{D D}$ for write operation.

\subsubsection{Bitline leakage}

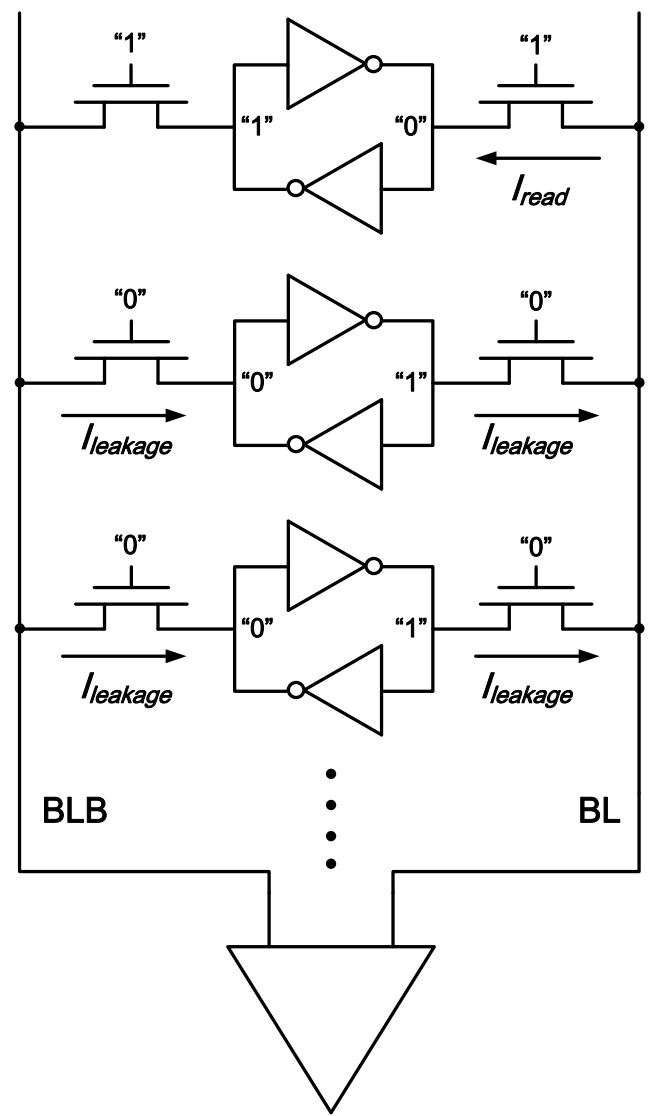

Figure 2.13 Worst case for bitline leakage [10] 
Another critical issue for subthreshold SRAM operation is the bitline leakage. In order to acquire good layout density, hundreds of cells must be connected to one bitline in the modern SRAM array. Assume that the memory cell in the first row with content ' 0 ' is the target cell to read. During the read operation, $B L B$ should be maintained high, while $B L$ should be pulled down to ground by ' 0 ' in the accessed node. Consider the worst case as shown in Figure 2.13. If all other cells connecting to the same bitline are ' 1 ', in this case, all access transistors except the target cell are closed. However, leakage current from the $B L B$ to $Q B$ tends to drive $B L B$ low and leakage current from $Q$ to $B L$ tends to pull $B L$ high. The more cells that are connected to each bitline, the higher the leakage current there will be. If variation caused by the leakage current on $B L$ and $B L B$ is too large, the potential on $\mathrm{BL}$ which should theoretically be ' 1 ' may be lower than the potential on $B L B$, which is expected to be ' 0 '. In this case, a read error occurs if $B L$ and $B L B$ fall into the undetermined region as shown in Figure 2.14 [11]. If no other leakage control technique is applied, a maximum of 64 cells could be attached to one bitline, as reported in [10].

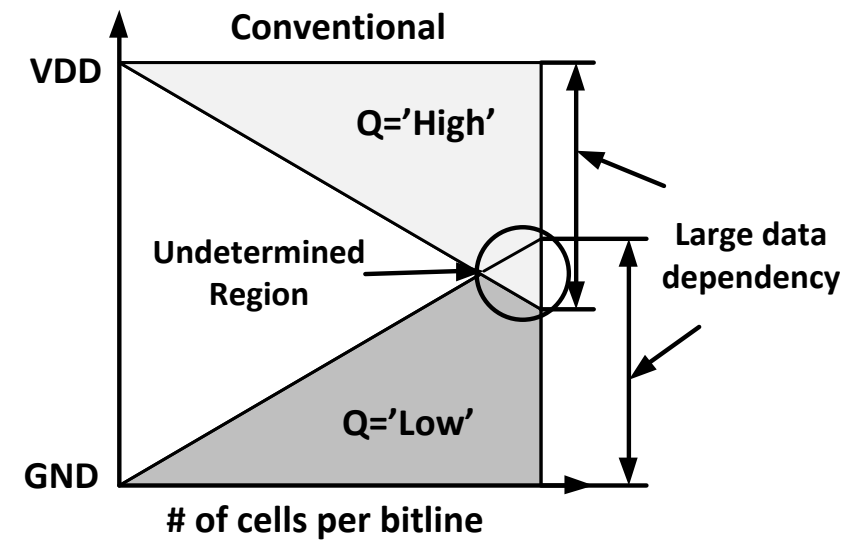

Figure 2.14 Large data dependency induced by bitline leakage [11] 


\section{Previous design to reduce bitline leakage}

\section{Kim's 10T cell [11]}

In Kim's 10T cell, one wordline and four more transistors (P3, N5, N6 and N7) are added, compared to a conventional 6T cell as shown in Figure 2.15. Similar to Chang's 8T cell, two write bitlines $B L$ and $B L B$, and one write wordline $W W L$, are used for the write operation; the read bitline $R B L$ and read wordline $R W L$ are used for the read operation. The write operation is the same as that of the conventional 6T cell, as well as Chang's 8T cell. For the read operation, when the read signal is enabled, $R B L$ is discharged according to the state stored in the cell. During the read cycle, $R W L$ is set to be high to turn on N6 and N7, and turn off $P 3$. It can be assumed that data stored in the cell is ' 1 ', that is $Q=$ ' 1 ', $Q B=$ ' 0 ', and in this case, $N 5$ is turned off. $R B L$ maintains to be precharged, while the access transistors ( $P 3$ and N5) are all off. Another condition is that if data in the cell is ' 0 ', that is $Q=$ ' 0 ' and $Q B=$ ' 1 ', and in this case, $N 5$ is turned on. The series access transistors $N 5, N 6$ and $N 7$ construct an open path from $R B L$ to ground to pull $R B L$ down.

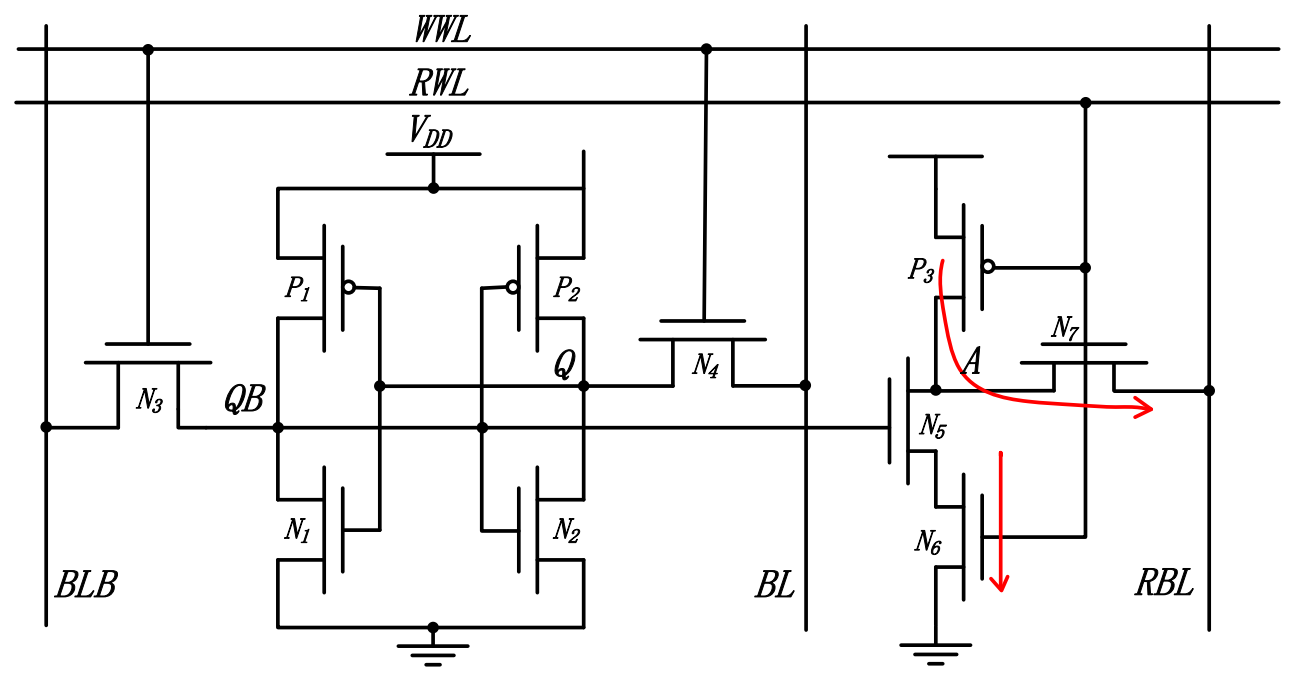

Figure 2.15 Schematic of Kim's 10T cell [11] 
Kim's $10 \mathrm{~T}$ cell eliminates the data dependent leakage by turning $P 3$ on when the SRAM cell is not accessed. In the idle status, $P 3$ is always on, thus the potential on node $A$ is ensured to be the same as $V_{D D}$ regardless the state in the cell. Assuming $R B L$ stays high during a reading cycle, and the potential on $R B L$ and node $A$ is the same, both equal to $V_{D D}$. If $R B L$ is pulled low by the leakage current from other cells on the same bitline, the leakage current from node $A$ to $R B L$ would pull $R B L$ back to high. Otherwise, if $R B L$ is expected to be pulled low, the leakage current from node $\mathrm{A}$ to $R B L$ will drive it high, regardless of the node states in the idle cells on the same bitline. The logic level on $R B L$ is determined by the pull-up leakage current from unaccessed cells and pull-down read current from the accessed cell. By doing this, data dependency on $B L$ and $B L B$ is reduced to a very small scale and a significant improvement for logic level ' 1 ' than the conventional 6T cell is shown in Figure 2.16. Test results demonstrate that Kim's 10T cell allows as many as 1024 bits per bitline.

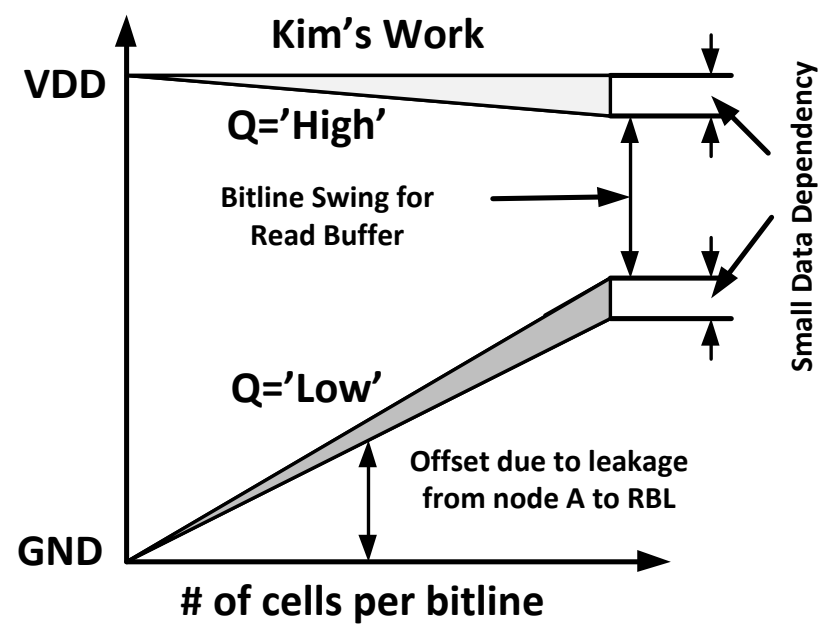

Figure 2.16 Improved small data dependency by bitline leakage [11] 


\section{Zero Leakage Read Buffer[10]}

In Verma's 8T cell design, the feet of all memory cells are connected to a read buffer instead of ground to reduce the leakage from standby cells, as shown in Figure 2.17. All cells in one row are connected to one read buffer foot, which is composed of an inverter. For the accessed row, the buffer foot is pull down to ' 0 ', and the read current is from $B L$, which is precharged to high, to the buffer foot. For all unaccessed rows, the buffer feet are pulled up to ' 1 '. Therefore, there is no leakage from $B L$ to the buffer feet, as the potential on the buffer feet and $B L$ are all high and the sole leakage source is the gate leakage and junction leakage from the read buffer feet.

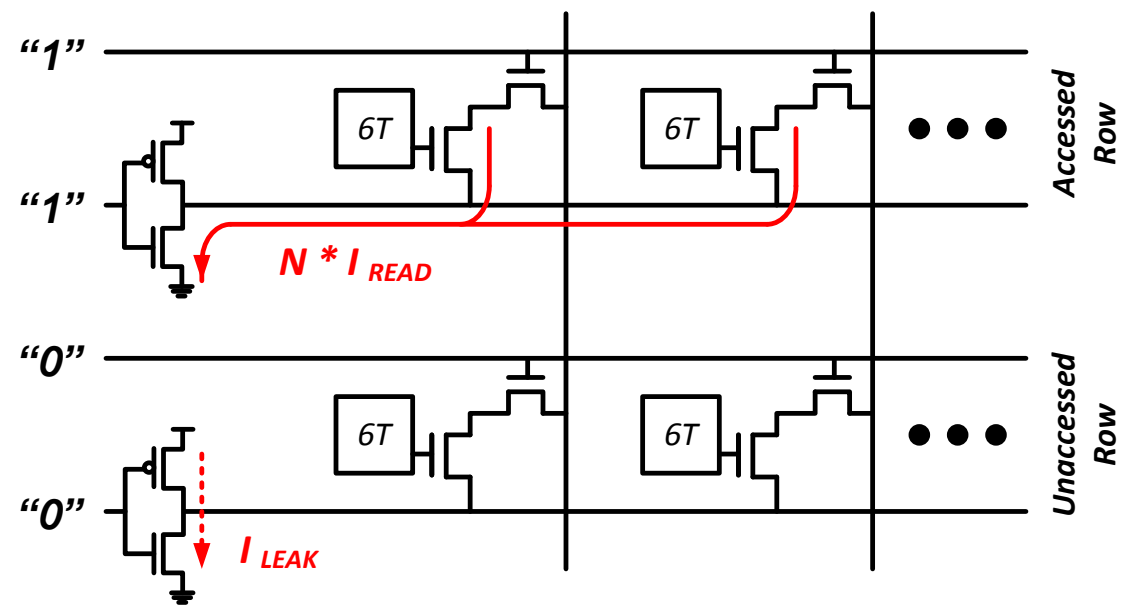

Figure 2.17 Leakage with read buffer feet structure

A notable drawback of this approach is that the buffer foot has to sink the read current from all the cells connected in one row, say $N \times I_{R E A D}$ in the figure, rendering the size of buffer foot impractically large if the number of cells per row is correspondingly large. On the other hand, an up-sized buffer foot increases leakage while decreasing density at the same time. To solve this problem, Verma et al. employed a charge-pump boost circuit to drive the buffer foot, as shown in Figure 2.18. This charge-pump circuit ensures $600 \mathrm{mV}$ instead of 
$350 \mathrm{mV}$ for the input of the buffer foot, named $B F B$ in the schematic, and increases the current by a factor of 500, which guarantees the minimum size of the buffer foot and small leakage [10].

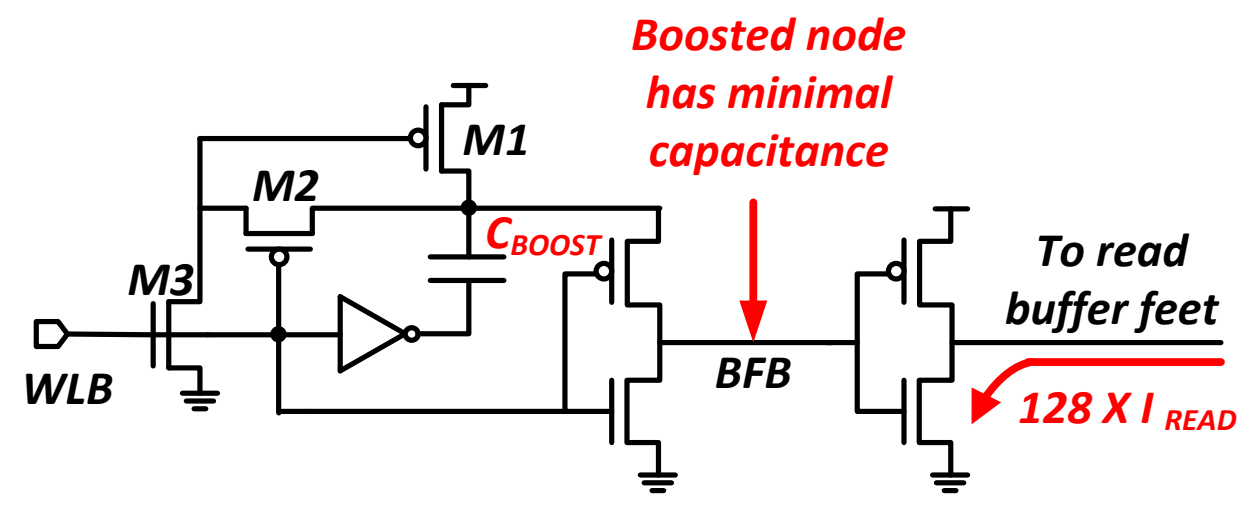

Figure 2.18 Boost circuit to drive read buffer [10]

\subsubsection{Low Writability}

Another challenge in subthreshold SRAM design is low writability. Some common techniques for improving the writability include:

1. Driving bitlines to a negative voltage

2. Raising the wordline voltage [26]

3. Lowering the cell $V_{D D}$ during writes [27]

All solutions above necessitate that the voltages must be raised or lowered in order to improve the writability without introducing a separate power supply. Reverse Short Channel Effect (RSCE) in subthreshold region was utilized in Kim's design [11]. As the Short Channel Effect $(S C E)$ is dominant in minimum channel length devices in strong inversion region, $R S C E$ is not a major concern in that region. However, because of the dramatically reduced Drain-Induced Barrier Lowering (DIBL), only RSCE is present in subthreshold region [28]. This phenomenon makes the threshold voltage $V_{t h}$ decrease monotonically, and 
the operation current increases exponentially as the channel length is longer. Simulation with a $130 \mathrm{~nm}$ technology demonstrates that, when $V_{D D}=1.2 \mathrm{~V}$, the maximum current through transistors decreases as the channel length increases. However, when $V_{D D}$ reduces to $0.2 \mathrm{~V}$, RSCE becomes dominant and the maximum current through the transistor occurs when channel length equals to $0.55 \mathrm{um}$ instead of $0.13 \mathrm{um}$ in the $130 \mathrm{~nm}$ technology.

\subsection{Fault-tolerant Design}

\subsubsection{Single Event Upset}

SEEs, caused by an energetic particle that penetrates sensitive nodes in IC materials, can lead to a plethora of adverse effects, from minor system responses to catastrophic system failures. A Single Event Upset (SEU) is a state change of storage cells caused by SEEs, which is a non-destructive soft error. In contrast to other circuits, memory cells can "remember" this kind of errors and the functionality of the whole system may eventually be affected. Therefore, SEUs must be carefully considered, especially for space applications where cosmic rays and high-energy ions are commonly present. When travelling to the earth's surface through the atmosphere, most of the cosmic rays and ions are trapped. However, a small amount of particles are able to penetrate the atmosphere and reach the surface of the earth. If high reliability is required, SEUs must also be taken into consideration. 


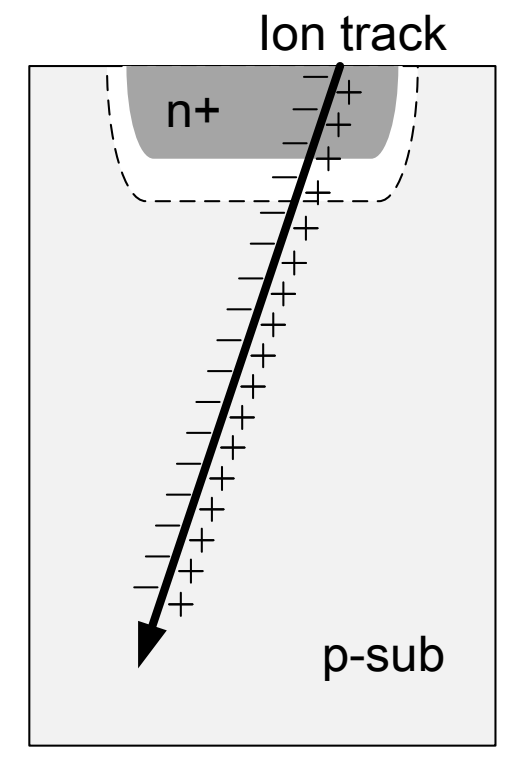

(a)

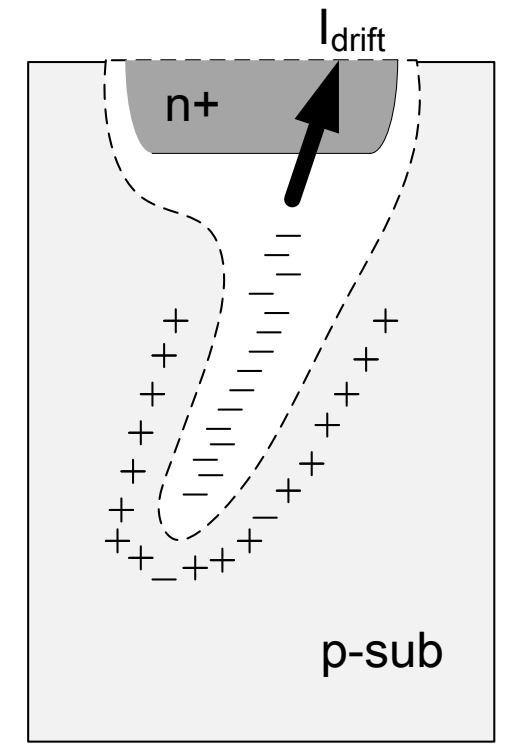

(b)

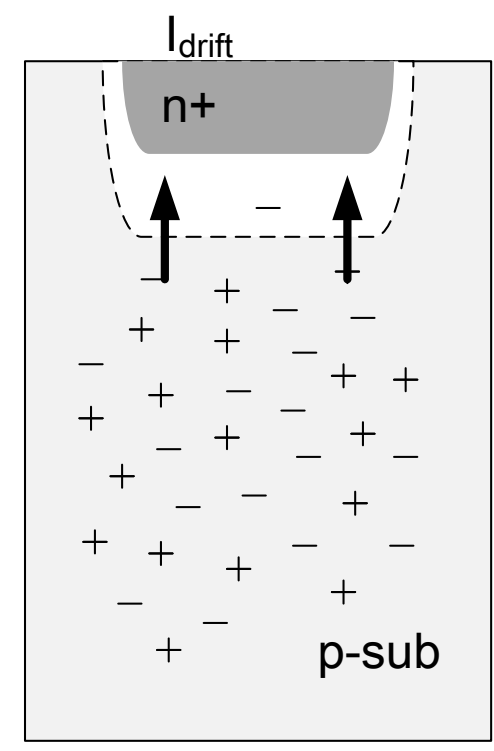

(c)

Figure 2.19 Mechanism of Single Event Effect [29]

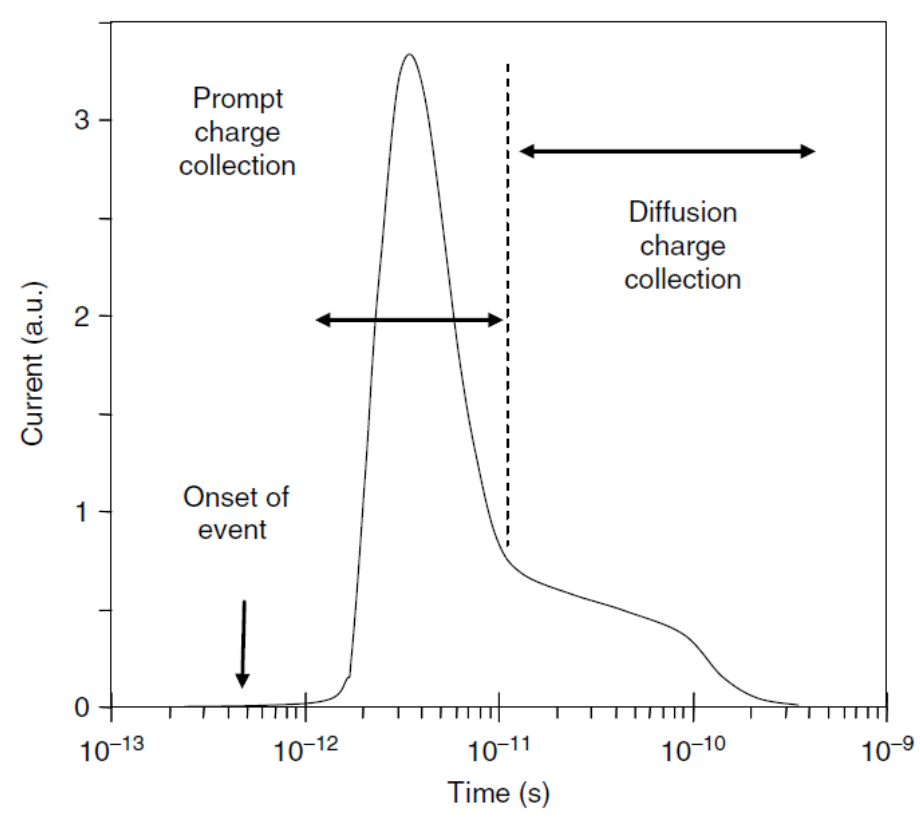

Figure 2.20 Single Event Effect current [29]

The mechanism for an SEE triggered by an ion track is as shown in Figure 2.19. At the time a heavy ion strikes through the diffusion area, a high concentration of electron-hole pairs is formed by the ionization effect (a). When the resultant track of carriers pass or get 
close to the depletion region, they will be collected rapidly by the electric field, leading to a large current transient. The depletion region extends deeply into substrate, which greatly enhances the charge collection efficiency (b). This rapid collection finishes within a nanosecond and then the diffusion becomes the dominance for charge collection. It takes a significantly longer time (hundreds of nanoseconds) for additional charge collection until all excess carriers are eliminated (c) [29]. Corresponding transient current caused by this progress is shown as Figure 2.20. The first half curve before the peak of the current is in correspondence with step (b). It is usually completed in the order of picoseconds. The other half after the peak of the current is for the step (c) and it is usually in the order of hundreds of picoseconds or nanoseconds.

\subsubsection{SEUs in SRAM}

To design fault-tolerant SRAM, the mechanism of SEUs in SRAM cell must be studied first. Previous researches have been carried out to characterize the mechanism of the SEU effect in SRAM [30]-[35].

One common accepted mechanism to characterize SEUs in SRAM is summarized by [35]. As shown in Figure 2.21, assure the data stored in the back to back inverter is '0', and thus, $Q$ is low and $Q B$ is high, $P_{1}$ and $N_{2}$ are on, $P_{2}$ and $N_{1}$ are off. The most SEU sensitive node is the reverse-biased node, namely $P_{2}$ and $N_{1}$ in these cross-coupled inverters. Another important consideration for charge collection is whether the node is located in a well or substrate [36]. The well-substrate structure provides a barrier that prevents carriers deposited in the deep substrate from diffusing back to the struck drain. In this case, the drain junction of $N_{1}$ is the most sensitive node for SEU effect, as $P_{2}$ lies in n-well substrate. 


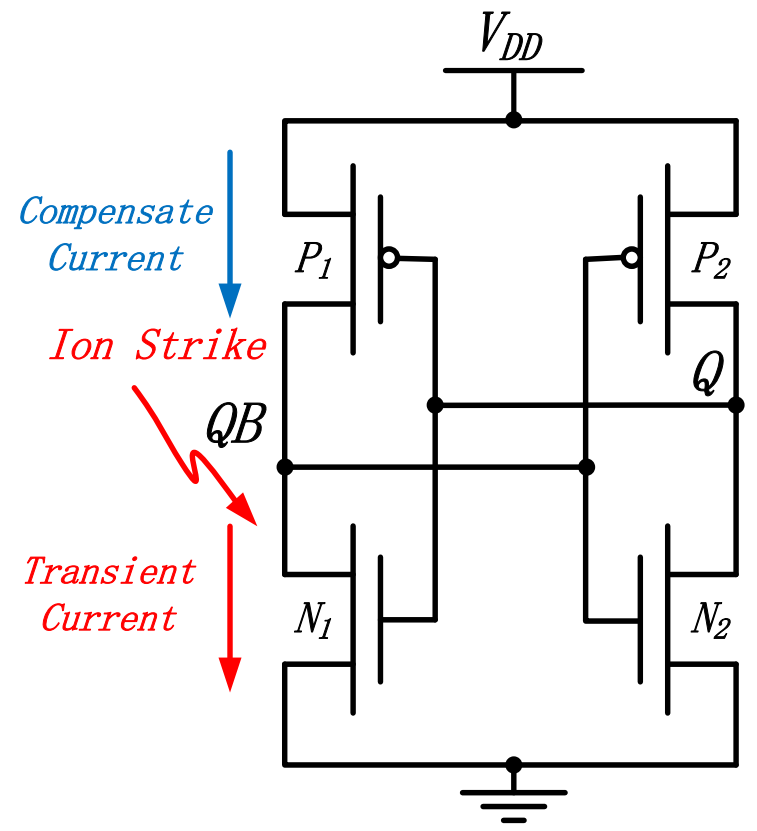

Figure 2.21 SEU in SRAM cell

Take pull down transistor $N 1$ for example, as shown in Figure 2.21; if heavy ion strikes on the drain area of $N_{l}$, electrons collected by the node results in a transient current flowing from $Q B$ to ground. To compensate for the current, the "on" restoring transistor $P_{2}$ generates a current from $V_{D D}$ to $Q B$ trying to recover the state. However, as the pull-up pmos is the weakest in the cell according to the cell constraint $C R$, it has only limited current drivability, which is referred to as finite channel conductance. Moreover, the voltage on $Q B$ is decreased and the voltage droop will be locked through the feedback between the cross-coupled inverters if it is large enough, subsequently causing the memory cell to be flipped. In this case, the error caused by the ion strike is "remembered" permanently. 


\subsubsection{Radiation Tolerant SRAM Design}

\subsubsection{Dual Interlocked Storage Cell}

To solve the SEU problem, many radiation-hardened SRAM structures are proposed [21], [22], [37], [38]. Among these designs, Dual Interlocked Storage Cell (DICE) [21] is the most commonly used one. The schematic of DICE cell is shown in Figure 2.22.

DICE cell employs two conventional cross-coupled inverter latch structures $(N 1, P 2)$ and $(N 3, P 4)$, which are connected as two bidirectional feedback inverters $(N 2, P 3)$ and $(N 4$, $P 1)$. Four nodes $A, B, C$, and $D$ in the cell are accessed simultaneously through access transistors (N5, N6, N7 and N8) for read and write operation. The immunity to SEUs for DICE cells relies on the dual node feedback control, which means that the state for each node is determined by both of the adjacent nodes. For example, node B is controlled by the two opposite diagonal (A-P2, C-N2).

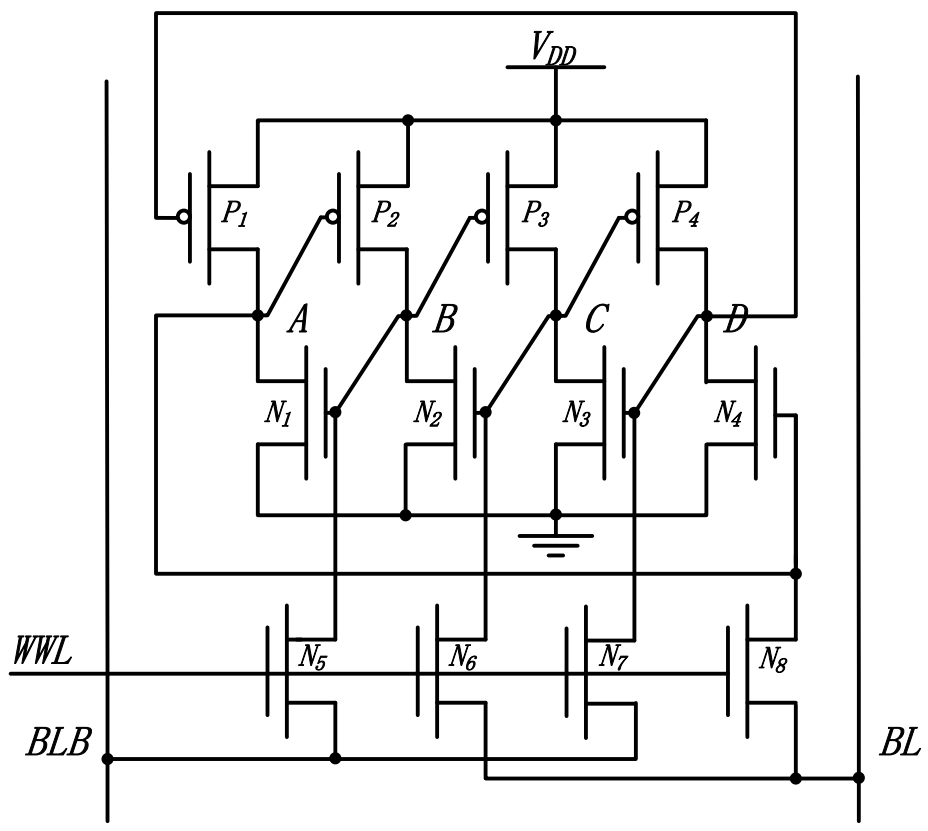

Figure 2.22 Schematic of DICE cell [21] 
Assume the initial value stored in the cell is ' 0101 ', that is $A={ }^{\prime} 0$ ', $B=$ ' 1 ', $C=$ ' 0 ', and $D=$ ' 1 '. If a particle strikes on the drain junction of $N 2$, a positive pulse current flowing from node $B$ to ground tries to pull down the potential on node $B$. The disturbance on node $B$ generates perturbation on node $C$ through the P-transistor feedback $P 3$, but it does not affect the node $A$ because the n-transistor feedback $N 1$ is blocked by the lowered voltage on node $B$. The perturbation on node $C$ does not further transfer to node $D$ and node $A$. Hence, logic disturbances are only constrained to node $B$ and $C$, and the perturbation is recovered when the transient current vanishes due to the restoring feedback by node $A$ and $D$ through $P 2$ and $N 3$. Simulation results for the SEU on DICE cell is illustrated in Figure 2.23.

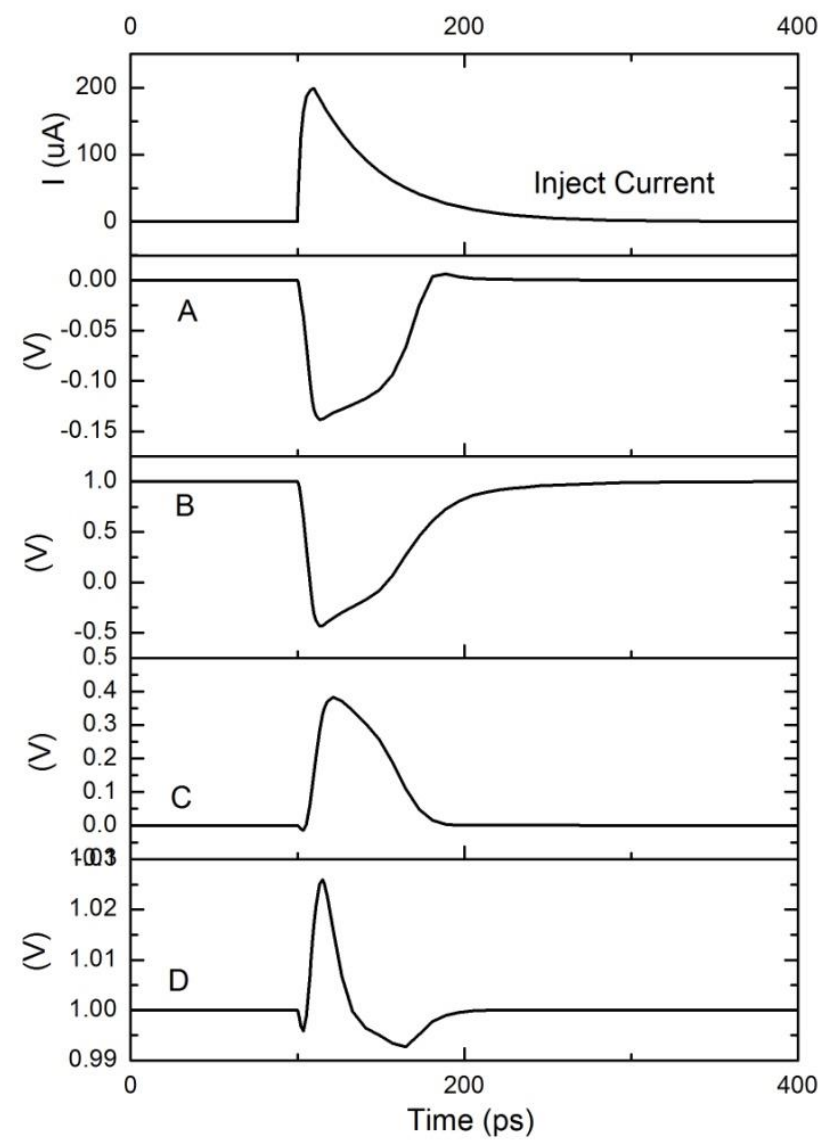

Figure 2.23 Simulation result of SEU effect on DICE cell 
Simulation and experimental results prove that DICE cell can recover from any upset as long as only one sensitive node is affected no matter how large the particle energy is. However, if two sensitive nodes with same logic state are hit simultaneously ( $A$ and $C$ or $B$ and $D$ ), the immunity is lost and the cell can be flipped. The chance of multi-node upset is very small and charge sharing is not a critical issue when the feature size of transistors is large, which is the case for less advanced technologies. However, with the technology scaling down, multi-node error becomes more serious as the charge generated by one hit might be shared by multiple nodes as the transistors size and space are both small.

\subsubsection{Quatro cell}

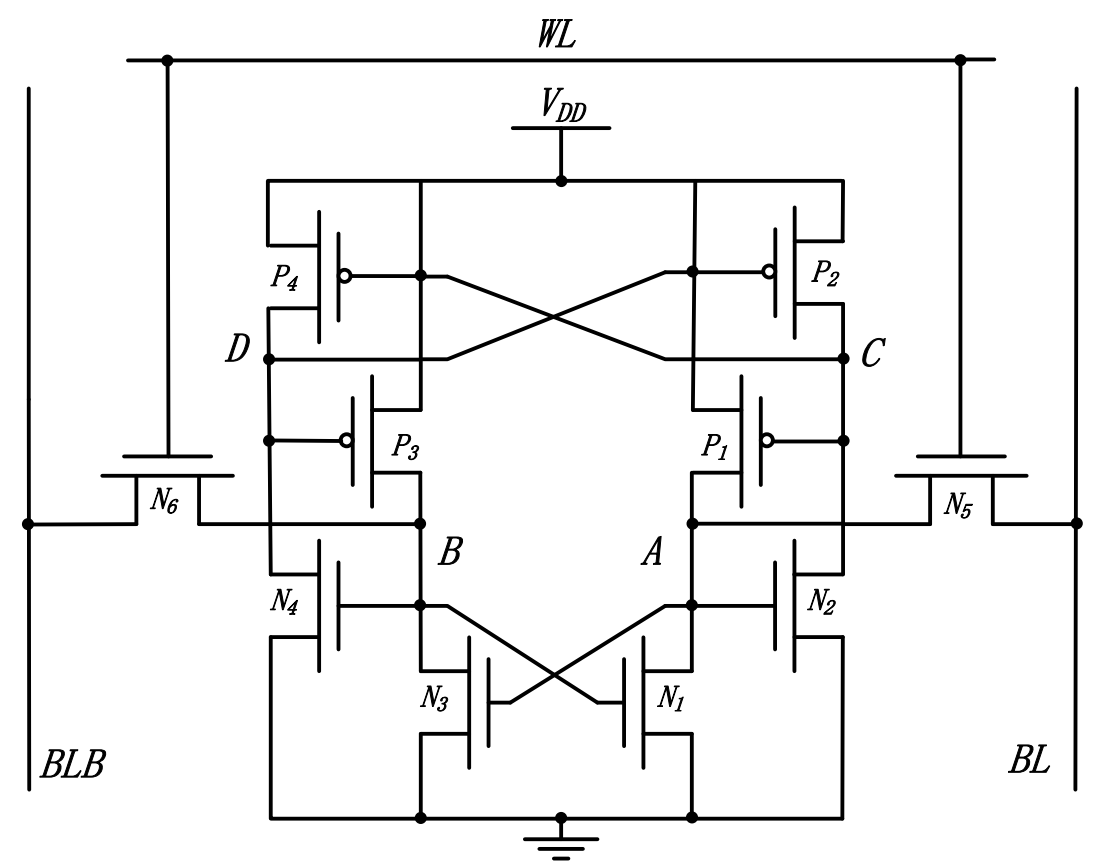

Figure 2.24 Schematic of Quatro cell [22]

Jahinuzzaman et al. proposed a 10-transistor radiation tolerance SRAM cell in 2009, as shown in Figure 2.24 [22]. Two access transistors ( $N 5$ and N6) are connected to the storage cell $A$ and $B$. The four nodes $A, B, C$ and $D$ are all driven by a pmos and nmos transistor, and 
the gates of the two transistors are driven by two different nodes. For example, node $A$ is driven by $P 1$ and $N 1$, the gate of $P 1$ is connected to node $C$, and the gate of $N 1$ is connected to node $B$. Node $A$ and node $B$ drive two nmos transistors $(N 2, N 3$ and $N 1, N 4)$, node $C$ and node $D$ connect to the gates of two pmos transistors $(P 1, P 4$ and $P 2, P 3)$. If data in the storage cell is ' 1 ', states of node $A, B, C$ and $D$ are ' 1 ', ' 0 ', ' 0 ' and ' 1 '. The following section outlines the read and write operations for the Quatro cell.

\section{Write Operation}

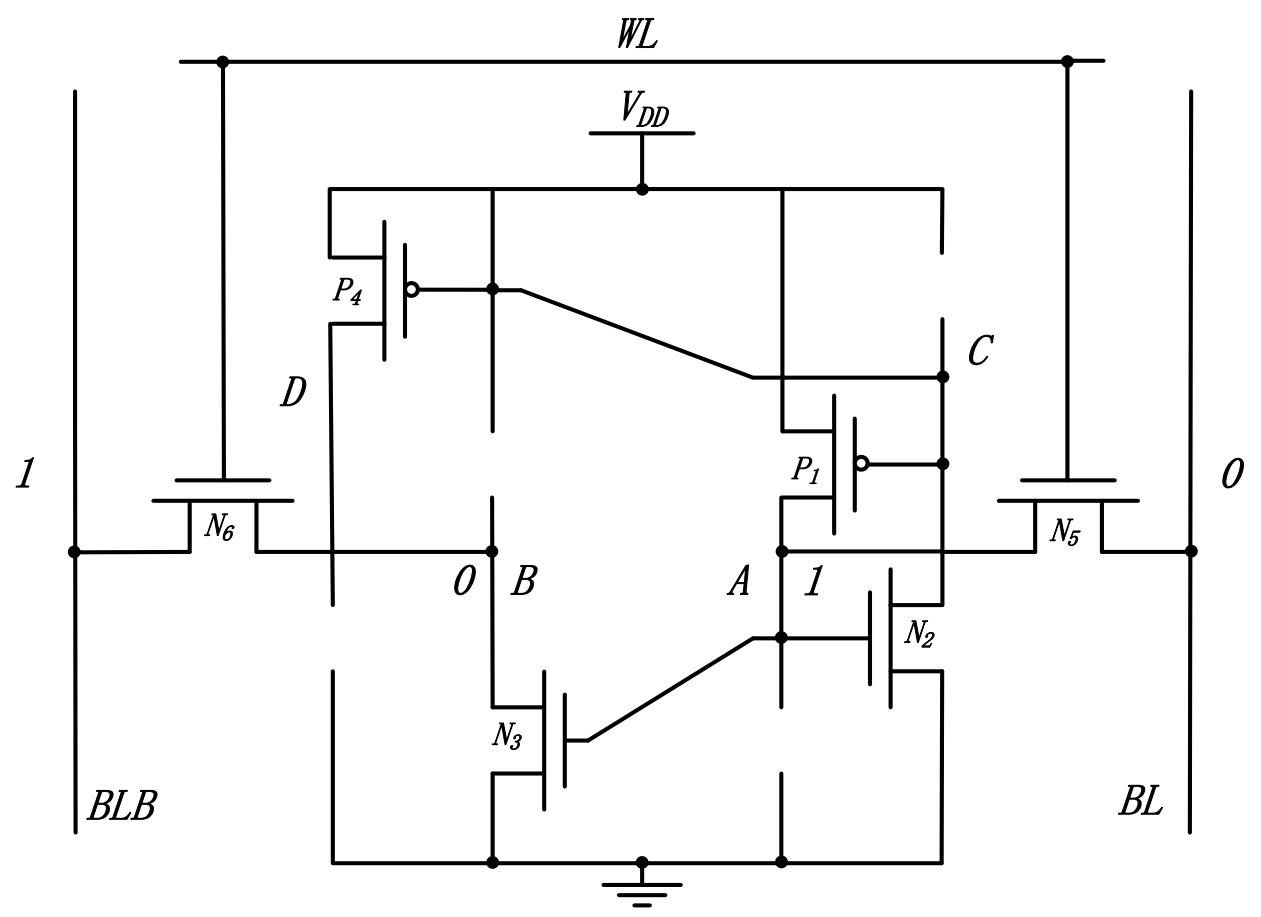

Figure 2.25 Simplified model for write operation

Again, assume that the initial state stored in the cell is ' 1 ', that is $A=$ ' 1 ', $B=$ ' 0 ', $C=$ ' 0 ', and $D=$ ' 1 '. Supposing that ' 0 ' is going to be written into the cell with original data ' 1 ', the initial state of the cell at the beginning of write process is simplified as shown in Figure 2.25. The $W L$ is set to high to turn on the access transistors $N 5$ and $N 6$. To turn off $N 2$ and $N 3$, node $A$ needs to be pulled down to be lower than $V_{t h}$ by $B L$. To achieve this, the drivability of 
$P 1$ must be weaker than N5, as the carrier mobility of nmos is higher. Pull up ratio $P R 1$, which is defined as $\frac{W_{P 1} / L_{P 1}}{W_{N 5} / L_{N 5}}$, can be 1 to pull node $A$ down below $V_{t h}$. Node $B$ is pulled up to $V_{t h}$ by $B L B$ to turn on $N 3$ and accelerate the pulling down of node $A$. To finish the write process, node $D$ must be lower than $V_{D D^{-}} V_{t h}$ to turn on $P 2$ and $P 3$, and thus, $N 4$ must be strong enough to fight against $P 4$. Pull up ratio PR2, which is defined as $\frac{W_{P 4} / L_{P 4}}{W_{N 4} / L_{N 4}}$, should be 0.75 or smaller in order for this operation to be completed reliably. Simulation waveforms of a write cycle are shown in Figure 2.26.

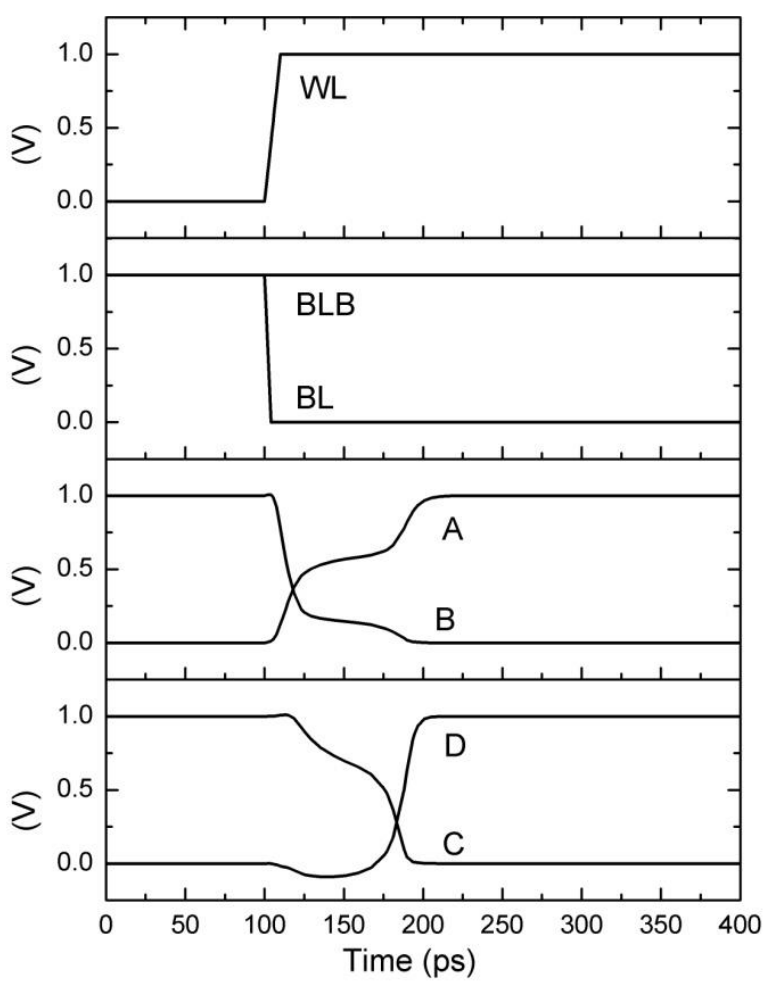

Figure 2.26 Simulation waveforms of the storage nodes, wordline, and bitlines in a write cycle

\section{Read operation}


In the read operation, assume data stored in the cell is ' 1 ', that is $A=$ ' 1 ' and $B=$ ' 0 '. The simplified model for the initial state during a read cycle is the same as in Figure 2.27. $B L B$ is driven low by node $B$ through the access transistor $N 6$. For a stable read operation, the pull down nmos transistor $N 3$ must be stronger than the access transistor $N 6$ to ensure node $B$ is not flipped by the precharged high voltage on $B L B$. The typical value for the aspect ratio defined as $\frac{W_{N 3} / L_{N 3}}{W_{N 6} / L_{N 6}}$ is $1.5 \sim 1.7$ for a safe read noise margin. Simulation waveforms of a read cycle are illustrated in Figure 2.28.

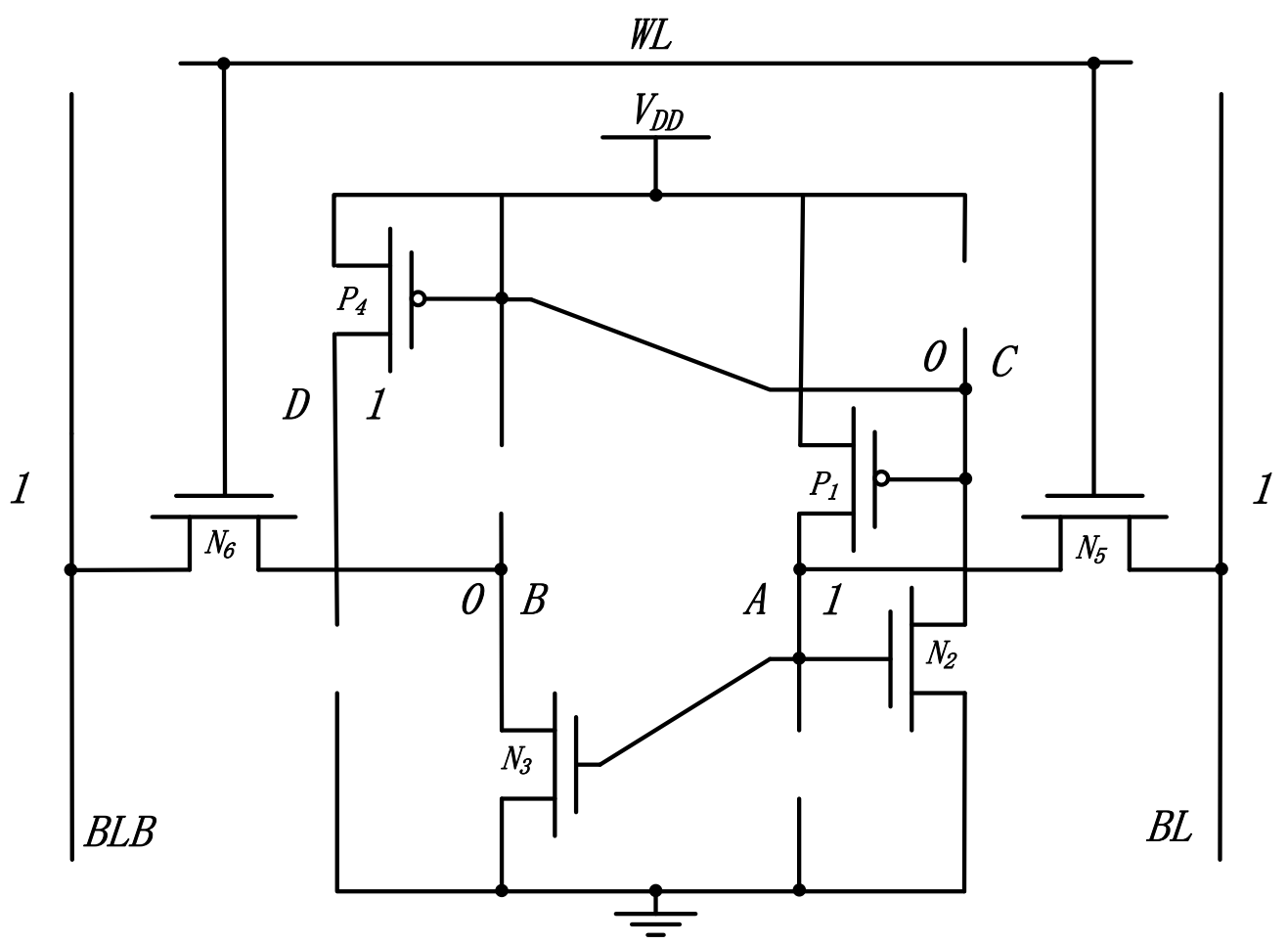

Figure 2.27 Simplified model for a write operation 


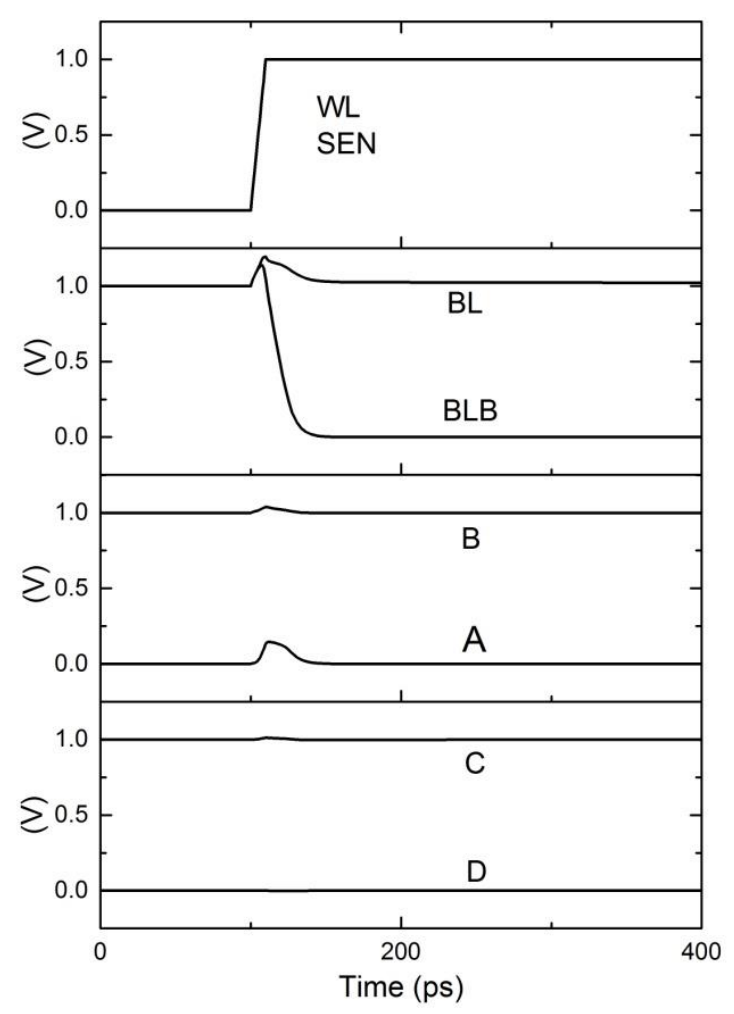

Figure 2.28 Simulation waveforms of the storage nodes, wordline, and bitlines in a write cycle

\section{SEUs in the Quatro cell}

The four nodes $A, B, C$ and $D$ are all driven by pmos and nmos transistors, and each transistor is correspondingly driven by two different nodes. If one node is flipped, it can be corrected by the nmos and pmos transistors driven by other unaffected nodes. As the cell is symmetric, only two nodes $A$ and $C$ will be analyzed to demonstrate the operations of the cell in an SEU event.

First, assuming node $A$ is flipped, there are two conditions to consider: 
1: If node $A$ equals to ' 1 ' and flips to ' 0 ': $N 3$ and $N 2$, which are driven by node $A$, are turned off. After the current transient, node $A$ is restored by the unaffected node $B$ and node $C$. Figure 2.29 illustrates the recovery for ' 1 ' to ' 0 ' flip at node $A$.

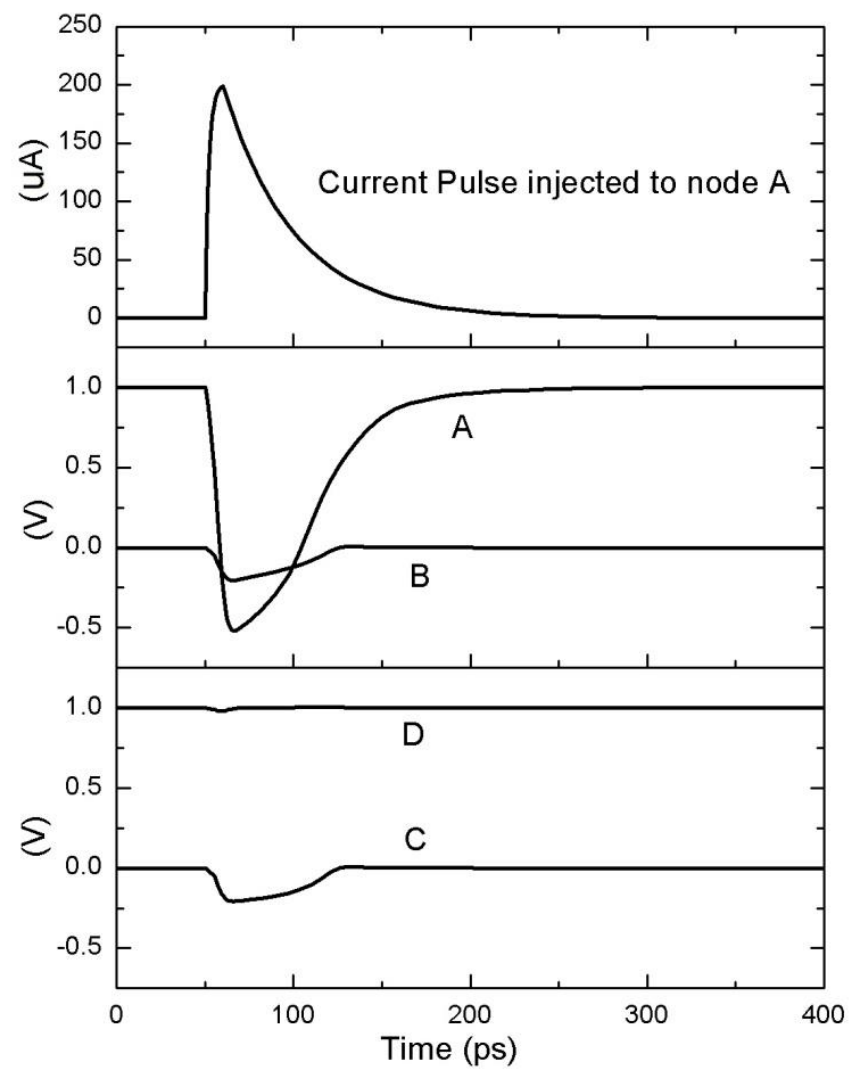

Figure 2.29 Recovery from injected current mimicking ' 1 ' to ' 0 ' at node A

2: If node $A$ is equal to ' 0 ' and flips to ' 1 ', $N 3$ and $N 5$ are turned on. The two transistor pairs $N 3$ and $P 3, N 2$ and $P 2$ fight each other to determine the states of node $\mathrm{B}$ and node C. From the ratio constraints listed above, the drivability of transistors is sorted by $P 3<N 6<N 3, P 2<N 2$, so $N 3$ wins the competition between $N 3$ and $P 3$ to flip node $B$ from ' 1 ' to ' 0 ', while $N 2$ beats $P 2$ to flip node C from ' 1 ' to ' 0 '. When $V_{C}$ and $V_{B}$ are pulled down to 
below $V_{t h}, P 4$ will be turned on and $N 4$ will be turned off. Eventually, node $D$ is pulled up to be ' 1 ' in order to complete the upset of the cell. However, the critical charge required for such flip is notably higher than the conventional 6T cell (3 times compared to the 6T cell), signifying that a high energy particle is required to flip the cell. The flip procedure for node $A$ from ' 0 ' to ' 1 ' is imitated by injecting exponential current to node $A$ from $V_{D D}$, as shown in Figure 2.30.

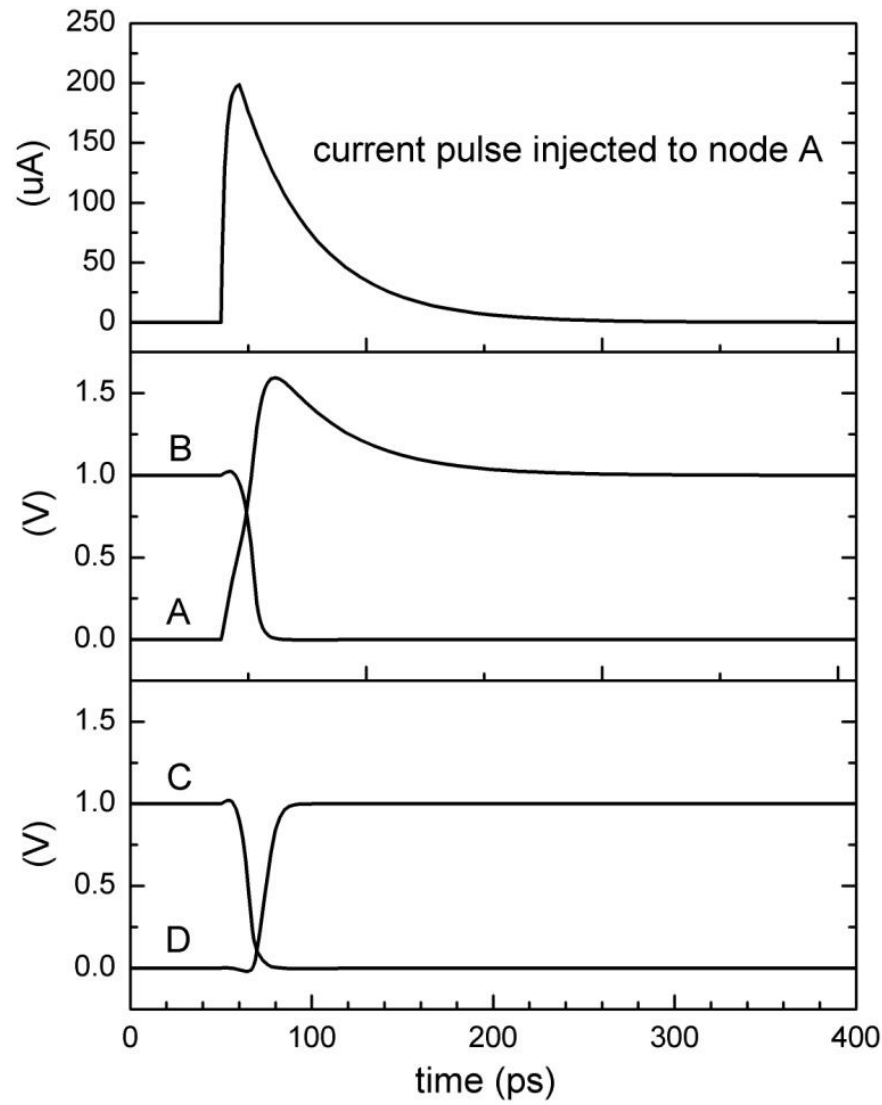

Figure 2.30 Flipping of cell by injecting current mimicking ' 0 ' to ' 1 ' at node $A$

Another node we need to analyze is node $C$, with a corresponding two assumptions to consider: 
1: If node $C$ is flipped from ' 0 ' to ' 1 ', and $P 1$ and $P 4$ are turned off, node $C$ will be restored by the unaffected node $A$ and node $D$ after the current transient. Therefore, flipping from ' 0 ' to ' 1 ' on node $C$ fails to flip the cell. Simulation results are illustrated in Figure 2.31.

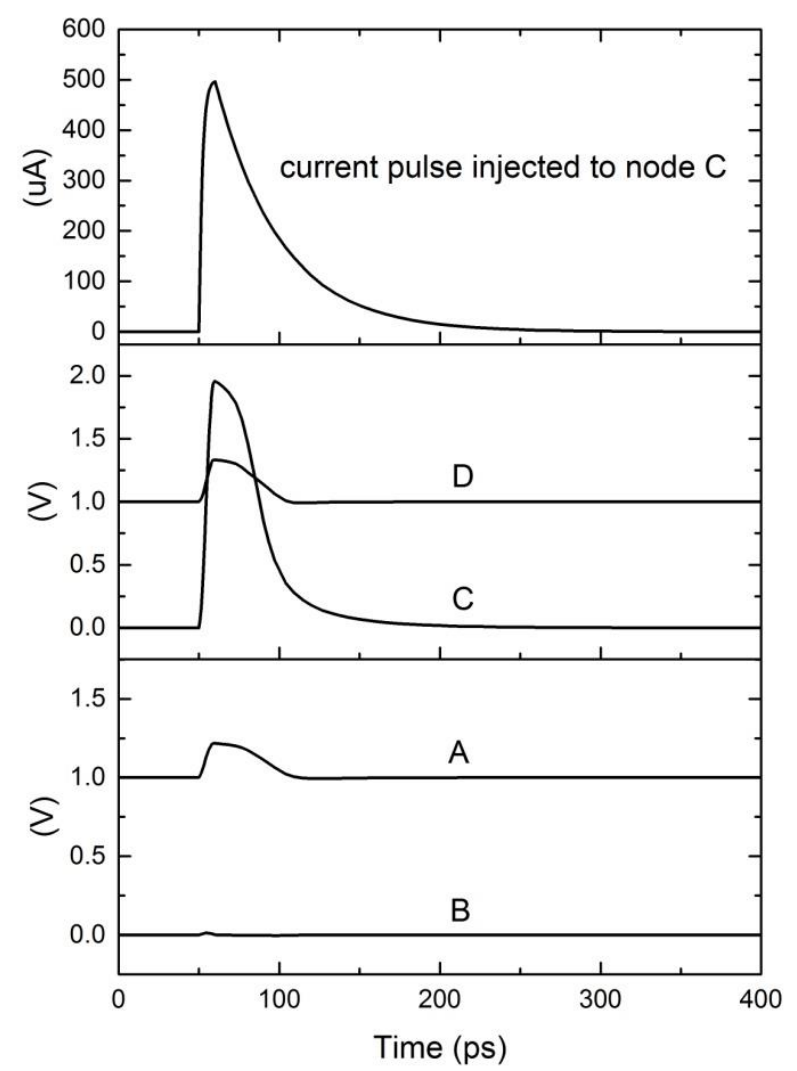

Figure 2.31 Recovery from injected current mimicking ' 0 ' to ' 1 ' at node $C$

2: If node $C$ is flipped from ' 1 ' to ' 0 ', and $P 1$ and $P 4$ that are driven by node $C$ are turned on, two pairs of transistors (P1 and N1,P4 and N4) compete with each other because they are both on. Node $D$ and node $A$ are pulled higher by the open access to $V_{D D}$. Although the drivability of transistors are $P 1<N 5<N 1, P 4<N 4$ from the size constraints described above, if the current pulse is big enough, $V_{C}$ can be pulled down to much lower than ground. 
In this case, $V_{A}$ and $V_{D}$ can be pulled up above $V_{t h}$. If $V_{A}$ and $V_{D}$ are higher than $V_{t h}, N 3$ is turned on and pulls the voltage on node $B$ lower. Decreased voltage on node $B$ further accelerates increasing potential on node $A$ and node $D$ to complete the flipping process. The energy required for SEU by flipping $C$ from ' 1 ' to ' 0 ' is much larger than conventional $6 \mathrm{~T}$ transistors by about 10 times, as demonstrated by the simulation results in Figure 2.32 .

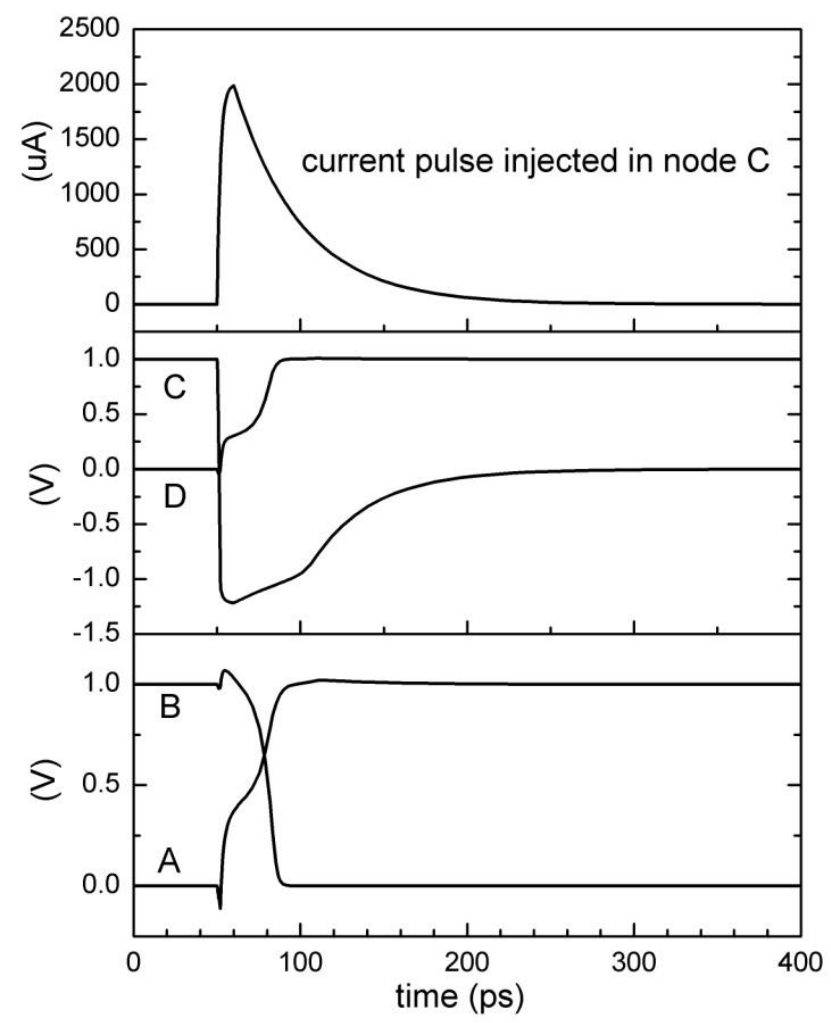

Figure 2.32 Flipping of cell by injecting current mimicking ' 1 ' to ' 0 ' at node $\mathrm{C}$ 


\section{CHAPTER 3}

SRAM DESIGN

In order to explore the power supply voltage dependence of heavy ion induced SEUs, a full custom designed $16 \mathrm{~Kb}$ SRAM test chip with four types of SRAM arrays was designed and fabricated in a 65nm, 9-metal technology. Among the four cells, 6T and 10T are chosen as unhardened cells, while Quatro and DICE are chosen as hardened ones to study their SERs at various power supply voltages. This chapter will introduce the SRAM design and configuration.

\subsection{Memory Design Overview}

The test chip contains four quadrants of 4k-bit SRAM cell array with one kind of memory cell in each. The target operating supply voltage ranges from $0.3 \mathrm{~V}$ to $1 \mathrm{~V}$. The block diagram of each page of SRAM is shown as such in Figure 3.1. 


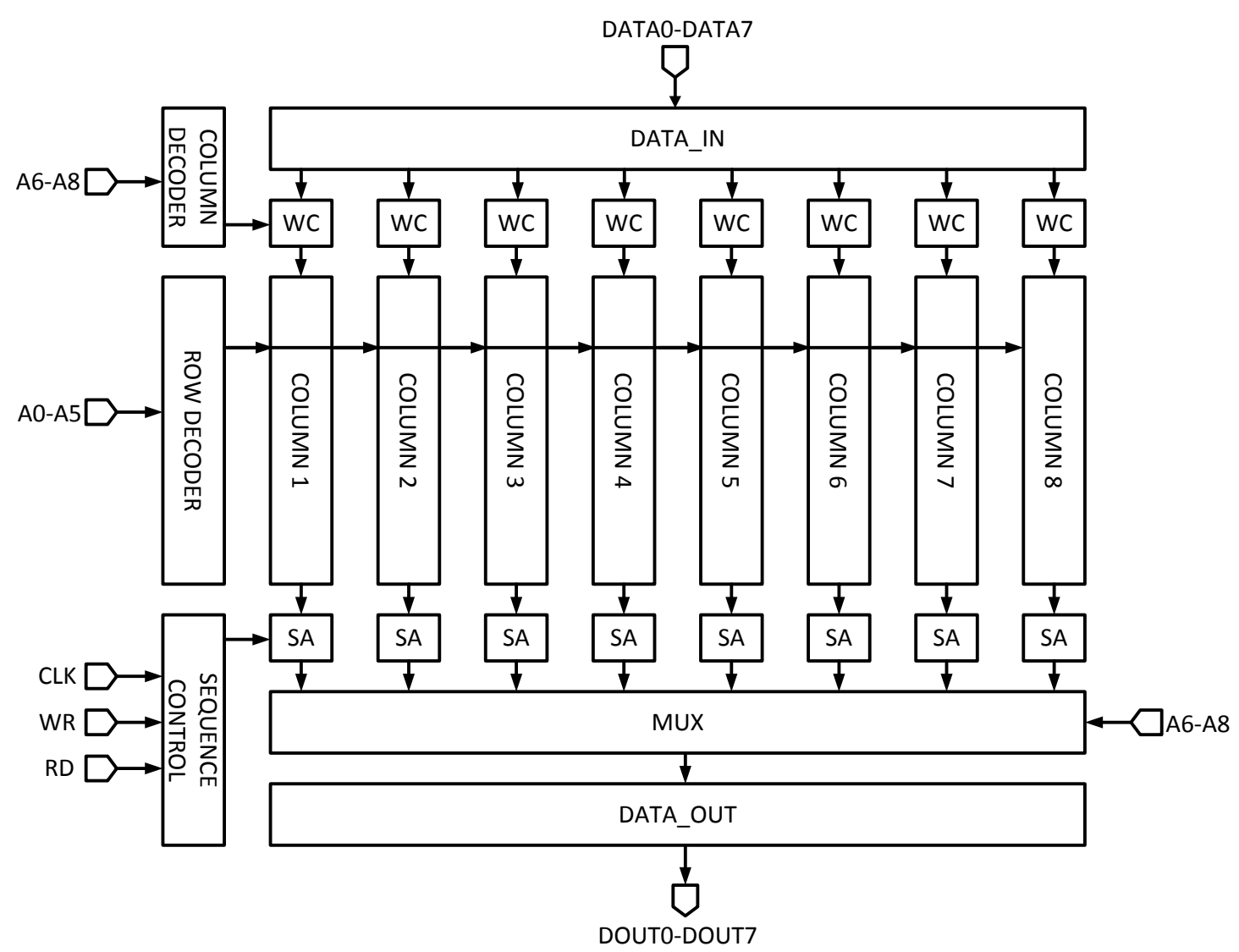

Figure 3.1 The block diagram for one page of the SRAM test chip

Each SRAM page contains eight columns of memory cell arrays, row decoder, column decoder, sequence control circuit, write circuit, sense amplifier (SA) and multiplexer (MUX). There are six inputs $(A O \sim A 5)$ for the row decoder, three inputs $(A 6 \sim A 8)$ for the column decoder, 8-bit inputs (DATAO DATA7), 8-bit outputs (DOUTO DOUT7), clock $(C L K)$, read enable $(R D)$, and write enable $(W R)$ signals. The corresponding functions are listed as follows.

Cell Array: The cell array contains eight columns with 64 bytes in each column, which makes $512 \times 8$ bits in one array. There are sixth-four cells connected to one bitline to reduce bitline leakage. 
Row Decoder: A six to sixth-four row decoder is used to decode the lower six address bits $(A O \sim A 5)$ to turn on the wordline of the selected row and turn off the wordline of all other unselected rows. There is one output (WL) or two outputs ( $R W L$ and $W W L)$ for each output of the row decoder, depending on the structure of the memory cells.

Column Decoder: A three to eight column decoder is employed to interpret the higher three address bits $(A 6 \sim A 8)$, enabling the column in which the target cell resides.

Sequence Control Circuit: It synchronizes all input signals, including $R D, W R$, address signals $(A O \sim A 8)$ and input data $(D A T A O \sim D A T A 7)$ by the clock signal. Read enable signal $(R D)$ and write enable signal $(W R)$ are all active low. It also generates all the other timing sequence signals needed for the read and write circuit.

Sense Amplifier: The dynamic latch-type sense amplifier with differential inputs is employed to distinguish and amplify the small swing of two bitlines to obtain the output quickly and accurately.

Write circuit: It receives the input data from input IOs and drives $B L$ or $B L B$ to complementary values to write into the memory cells.

Multiplex: Eight multiplexes decode the higher three address bits $(A 6 \sim A 8)$, choosing the output from eight columns to output IOs.

\subsection{Memory Cells and Peripheral Circuits Design}

This chapter will provide detailed design information for each part aforementioned in the previous section. 


\subsubsection{Memory cell design}

As mentioned above, four kinds of different memory cells are adopted in the test chip. They are the conventional 6T cell, 10T cell, modified DICE cell and Quatro cell. Among these four memory cells, two cells are radiation-hardened (modified DICE cell and Quatro cell) and three cells are suitable to be operated in sub-threshold voltages (10T cell, modified DICE cell and Quatro cell). The radiation hardness and sub-threshold operation characteristics of these SRAM cell arrays are listed in Table 3.1.

Table 3.1 Characteristics of SRAM Cells

\begin{tabular}{|c|c|c|}
\hline SRAM & Radiation-Hardness & Sub-threshold Aware \\
\hline $6 \mathrm{~T}$ & no & no \\
\hline 10T & no & yes \\
\hline Quatro & yes & yes \\
\hline DICE & yes & yes \\
\hline
\end{tabular}

\subsubsection{Conventional 6T cell}

The schematic, along with the writing and reading operation of the conventional 6T cell, is given in chapter 2.1. Recall the schematic of the conventional 6T cell in Figure 2.1; the conventional $6 \mathrm{~T}$ cell is neither low-power operated, nor radiation tolerant. However, as a standard and most commonly used SRAM cell, the 6T cell is employed for comparison purposes. 


\subsubsection{10T cell}

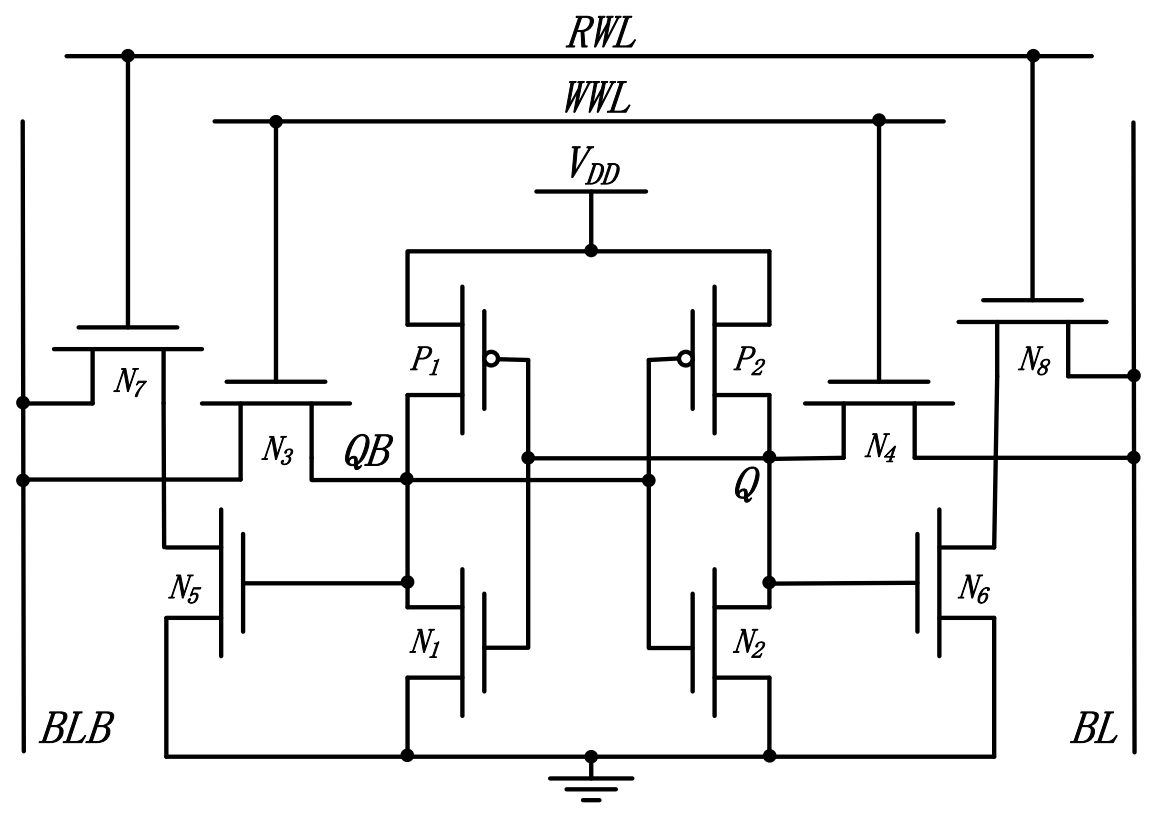

Figure 3.3 Schematic of 10T cell

The schematic of 10T cell is outlined in Figure 3.3. Similar to the 8T cell in Chapter 2.2.1, two more transistors are added on each bitline to isolate the read and write operation. Rather than introducing a separate read bitline, the 10T cell uses the same bitlines with write operation. For read operation, WWL is low and write access transistors $N 3$ and $N 4$ are off. Subsequently, both $Q$ and $Q B$ are connected to the bitline through series read transistors (N5, $N 7$ and $N 6, N 8$ ) in order to benefit from the sense amplifier, which can sense and amplify small differences of two bitlines. In this case, the read signal noise margin is increased to the same value as the hold signal noise margin, rendering the 10T cell low voltage operational. Analogous to the conventional $6 \mathrm{~T}$ cell, the $10 \mathrm{~T}$ cell is also not radiation tolerant. As a standard ultra-low power SRAM cell, the 10T cell is employed for comparison purposes to a hardened cell with a different supply voltage. 


\subsubsection{Modified DICE cell}

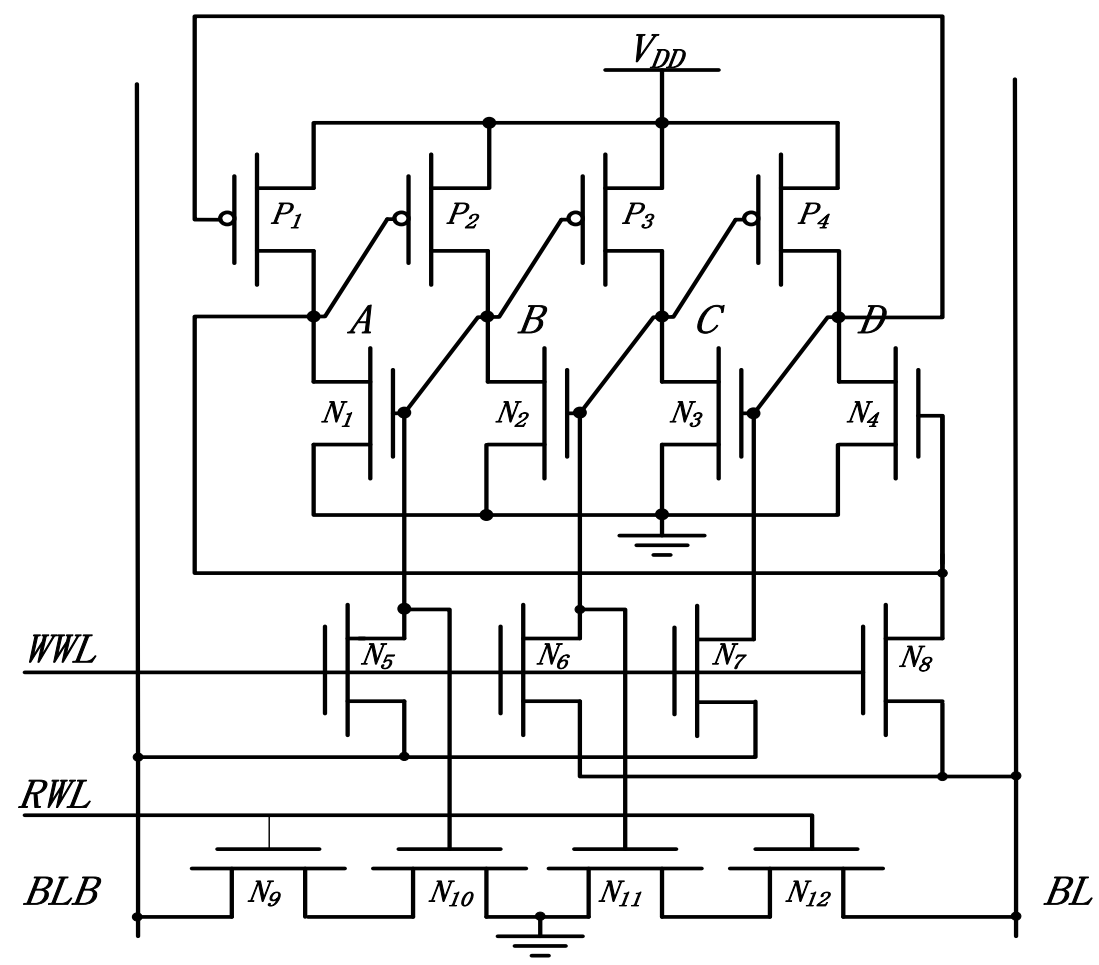

Figure 3.4 Schematic of low power operational DICE cell

Recall the schematic of DICE cell in 2.3.3.1. Analogous to the conventional 6T cell, the traditional DICE cell is not sub-threshold operational. To make the DICE cell functional with a sub-threshold voltage, the proposed modified DICE cell adds four transistors (N9, $N 10, N 11$, and N12) and one separate read word line $(R W L)$ to isolate the read and write operations. For the write operation, the procedure is the same as the traditional DICE cell. For the read operation, assume the data stored in the cell is ' 1010 ', that is $A=$ ' 1 ', $B=$ ' 0 ', $C=$ ' 1 ', and $D={ }^{\prime} 0$ '. During the read cycle, the write wordline ( $\left.W W L\right)$ is set low to turn off the access transistors $(N 5, N 6, N 7, N 8)$, and the read word line $(R W L)$ is set to high to turn on $N 9$ and N12. N1O and N11 are driven by node $B$ and node $C$, and in this case, N1O is off and N11 is on depending on the node states of $B$ and $C$. Furthermore, the left path from $B L B$ to ground 
through $N 9$ and $N 10$ is blocked by $N 10$, and $B L$ is pulled down due to the open path from $B L$ to ground through N11 and N12 at the right side. Subsequently, the potential difference between $B L$ and $B L B$ will be set up. It should be noted that the precharged high voltage on $B L$ and $B L B$ does not affect the state on node $B$ or node $C$ during the read operation, as they are connected to the gates of access transistors. Thus, the read noise margin for the modified DICE cell is the same as the hold noise margin, ensuring that it functions at sub-threshold power supply voltages.

The DICE cell is tolerant to SEUs, as it can recover from any single node upset regardless of how large the energy is. However, if more than one node is affected, the cell can be flipped. In the less advanced technologies, the probability of two particles striking at two different nodes in one cell at the same time is rare. However, as transistor size is continuously scaled down, the charge generated by one strike can be spread and absorbed by more than one node. To avoid this kind of charge spread, one can try to separate sensitive pairs far from each other by employing a layout strategy. 


\subsubsection{Quatro Cell}

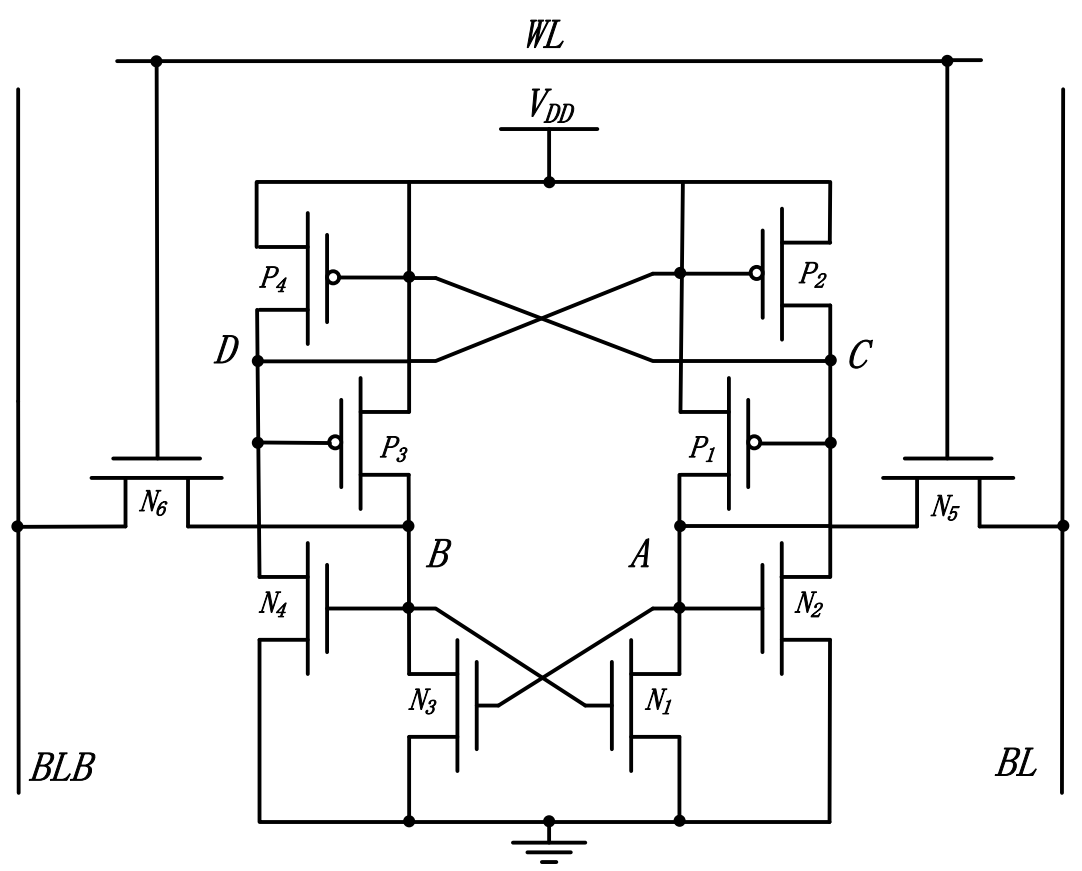

Figure 3.5 Schematic of an Quatro cell

Recall the Quatro cell schematic in Chapter 2.3.3.2, Quatro cells are SEU tolerant, as discussed in chapter 2. Moreover, the read noise margin of the Quatro cell is larger than that of the 6T cell, rendering the Quatro cell an appealing option for low power applications. In recent years, particle radiation experiments (neutron, alpha, and heavy ions) have been carried out and the results have demonstrated higher radiation tolerance of Quatro compared to that of DICE when they were both used to construct flip-flops in a 40nm technology [39]. In this case, Quatro cells were adopted in this work and experiments were carried out to study the radiation effects of Quatro cell with different power supply voltages.

\subsubsection{Address Decoders}

The capacity of the SRAM is $4 \mathrm{~K}$ bits per cell array, so 11 addresses $(A O \sim A 10)$ are used. For each SRAM array, six addresses $(A O \sim A 5)$ are used for the row decoder, which 
decodes the row address signals and controls the wordline for each row. There are two stages in the row decoder circuit. Stage one is a 6-to-64 decoder, which includes six lower address inputs $(A O \sim A 5)$ and 64 outputs, each of the outputs is connected to stage two - wordline generation circuit. The wordline generation circuit produces either one wordline $(W L)$ or two wordlines ( $R W L$ and $W W L)$, depending on the memory cell structure in the array.

Global wordline, used in the normal SRAM structure, decreases the hold noise margin of unselected memory cells to the same value of the read noise margin. This drawback is referred to as the pseudo-read problem. For all the idle cells in the same row with the target cell, the high global wordline turns on the access transistors of the cells in hold state, and the precharged high voltage on $B L$ and $B L B$ makes the situation the same as the beginning of a read cycle, as illustrated in Figure 3.6. The pseudo problem can be solved by adopting the local wordline, as demonstrated in Figure 3.7. In Figure 3.7, it is apparent that only the local wordline of the selected column is high, and all the access transistors in hold state are turned off. In this case, the high voltage on $B L$ and $B L B$ does not affect the node state in the unselected memory cells.

Another decoder in the SRAM is the column decoder, for which three addresses (A6 A8) are used as inputs. The column decoder is used to generate the column select signal to enable and disable the local wordline and write control circuit. If the column is accessed, the column select signal which is the output of column decoder will be set to high, while all other columns are low. In this case, only the local wordline of the accessed column is enabled to avoid the pseudo-read problem. In addition, adopting the local wordline reduces the load on the wordline, thus saving energy. 


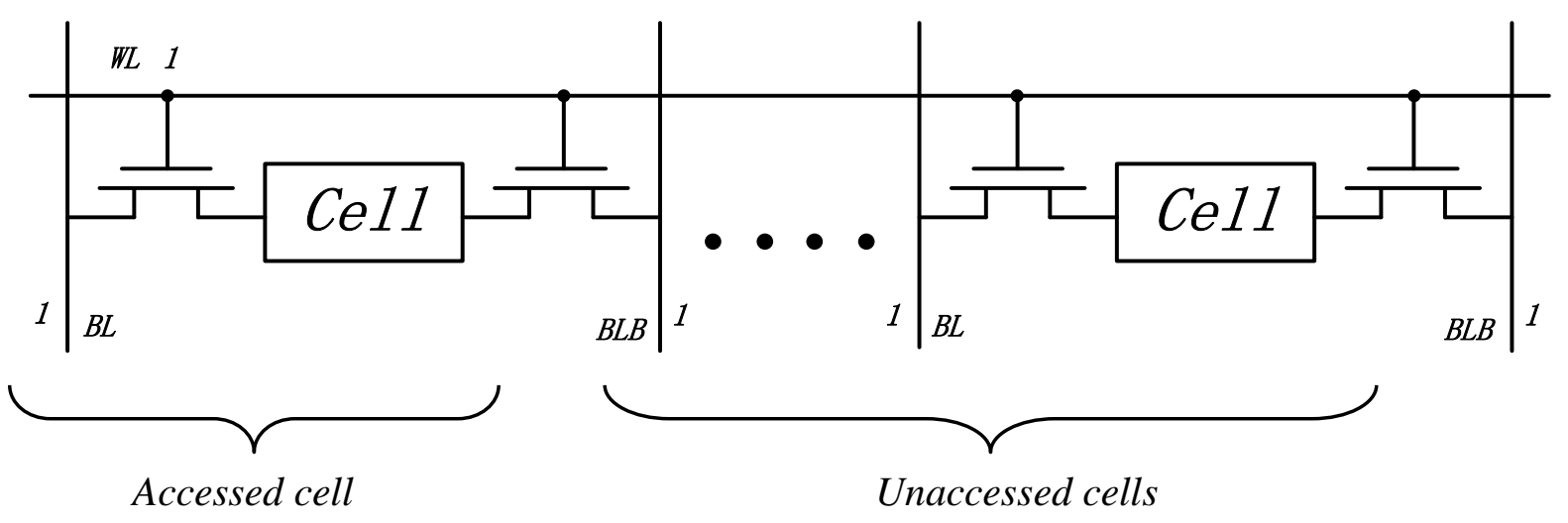

Figure 3.6 Pseudo read problem of hold cells

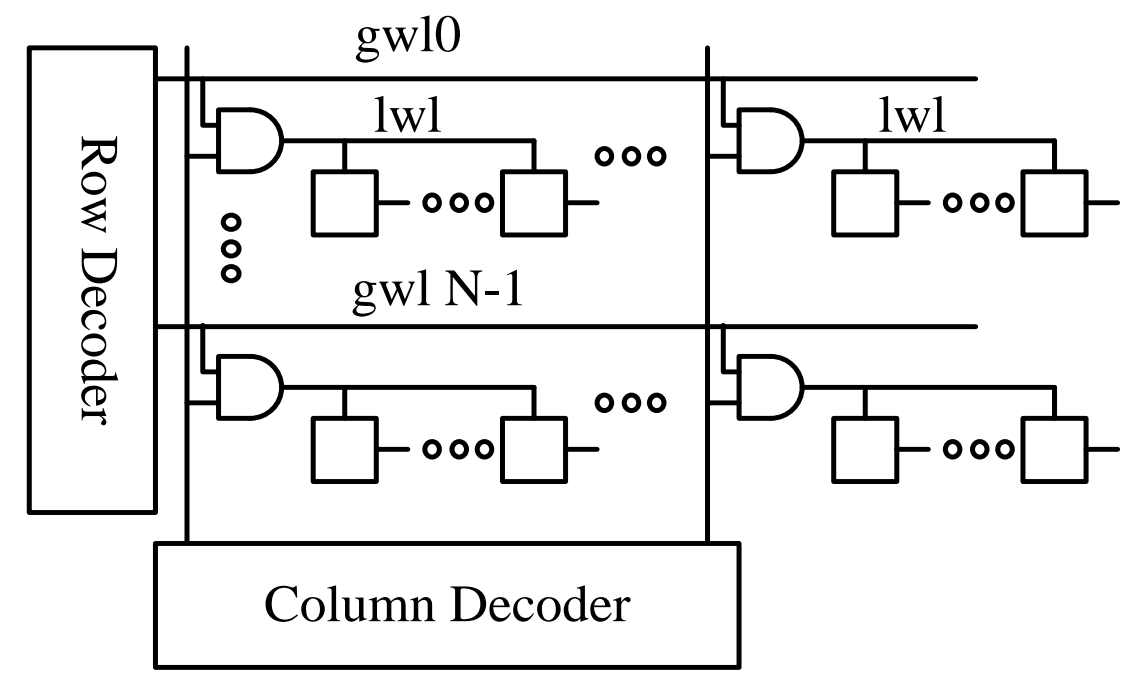

Figure 3.7 Global wordline and Local wordline [24]

\subsubsection{Multiplexer}

As there are eight columns with one-byte outputs in each column, an eight-to-one multiplexer is adopted to choose the correct output from total eight-bytes outputs. The inputs for the multiplex are $A 6, A 7$ and $A 8$. Since there are four memory quadrants in the design, four to one array-select multiplexer that decodes the highest two address bits $(A 9, A 10)$ is 
employed to choose the right quadrant of SRAM to read from. The schematic of the four to one multiplexer is illustrated in Figure 3.8.

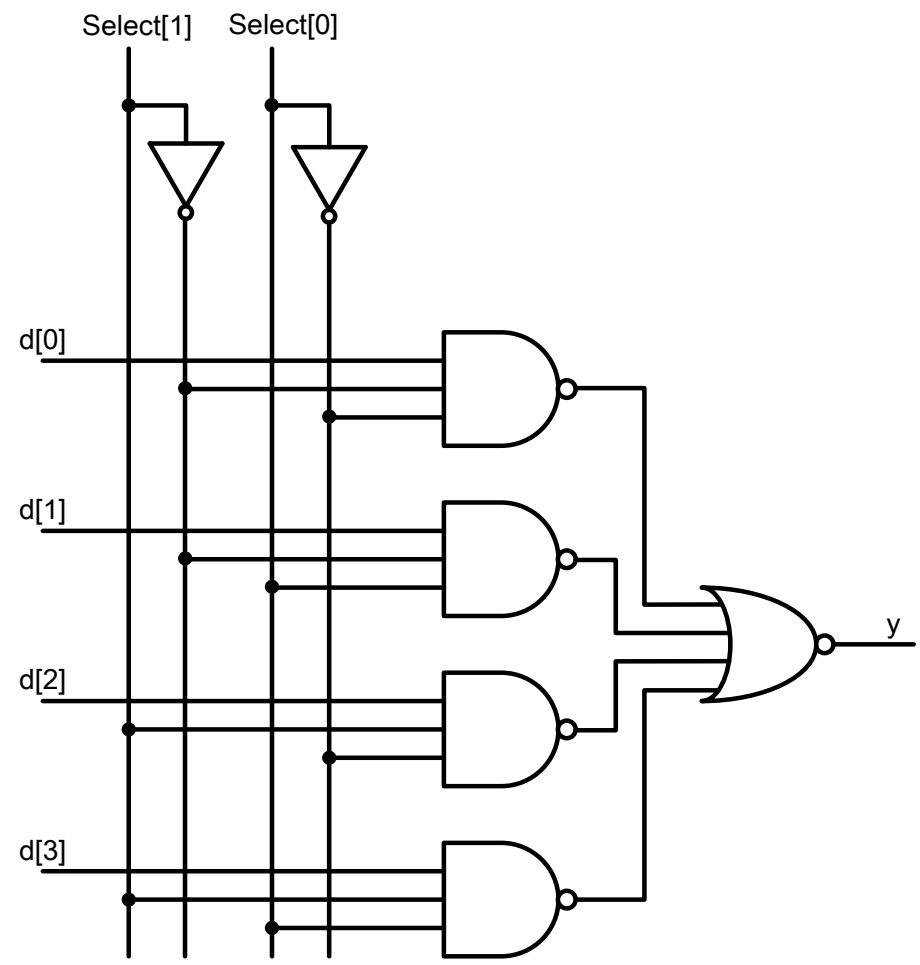

Figure 3.8 Schematic of four to one multiplexer

\subsubsection{Bitline Conditioning}

Bitline conditioning circuit is used to precharge the bitlines ( $B L$ and $B L B$ ) to a high voltage prior to the write operation and during idle state. One simple bitline conditioning circuit uses a pair of weak pull up p-transistors, as outlined in Figure 3.9.

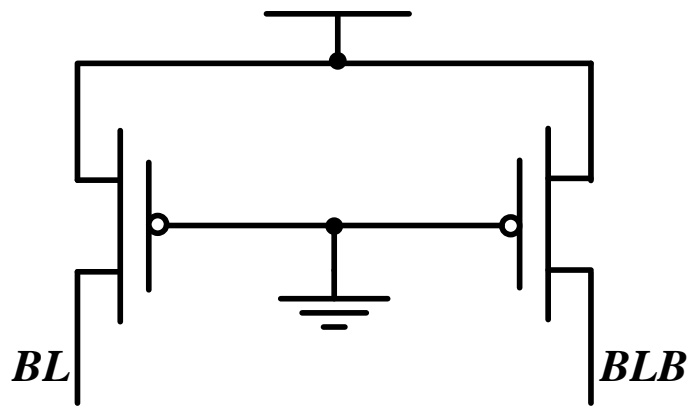

Figure 3.9 Bitline conditioning circuit 
However, because the p-transistors are always on, there is pull-up current from $V_{D D}$ which tends to drive $B L$ and $B L B$ high. In this case, the read operation is slowed down, and the bitline that should be pulled down to ground by memory cells during the read operation cannot reach ' 0 '. This situation is serious, especially when the supply voltage is lowered down to sub-threshold region. Therefore, the "always on" bitline conditioning circuit is not an optimal choice for the sub-threshold SRAM design. In order to make the circuit suitable for low-power operation, rather than always on, two weak p-transistors are driven by a column controlled signal $\Phi$ as shown in Figure 3.10. For the selected column, the p-transistors are off during the read operation and the first half clock cycle of the write operation. Otherwise, the p-transistors are on to keep both bitlines high. The timing sequence of $\Phi$ is outlined in Figure 3.11.

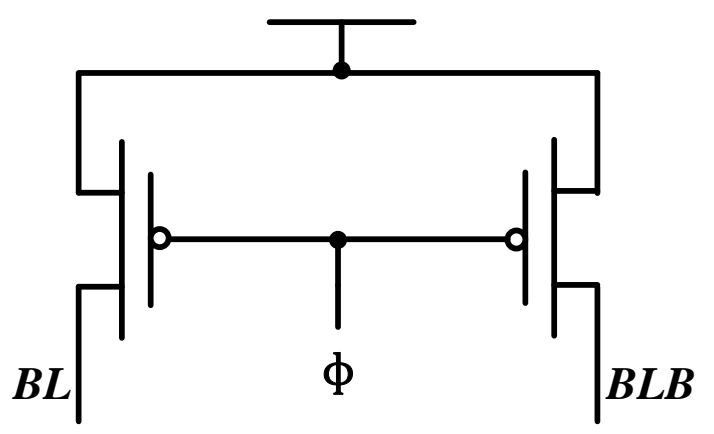

Figure 3.10 Controlled bitline conditioning circuit 


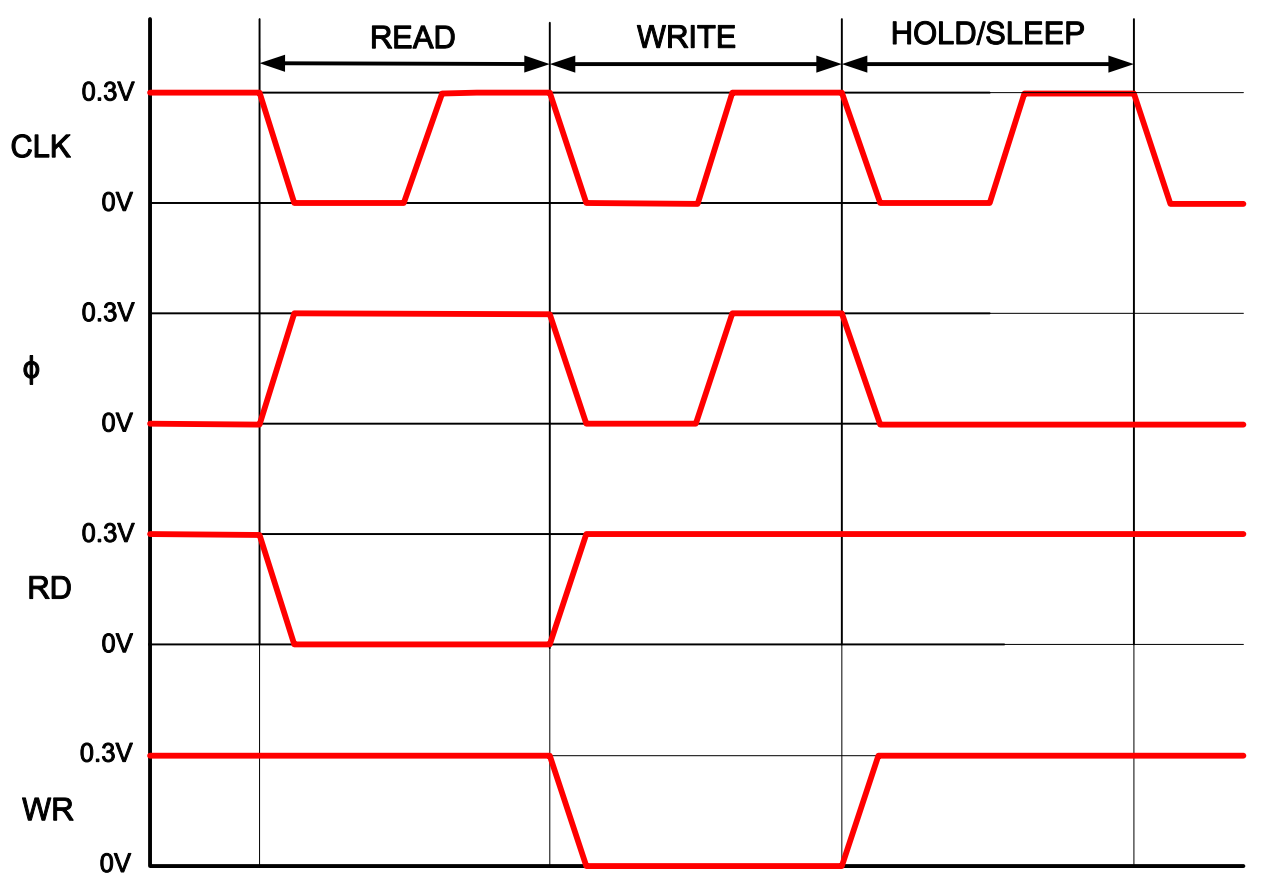

Figure 3.11 Timing sequence of $\Phi$ during read, write and hold operations

\subsubsection{Sense Amplifier}

In the SRAM design, both large-signal and small-signal sense amplifiers can be utilized. An inverter-type read buffer can be used as a large-signal sense amplifier. However, it requires a relatively large swing on bitline, leading to a prolonged delay and a more sizable power consumption, as this swing must be larger than $V_{t h}$. Therefore, the small-signal sense amplifier is preferred in fast SRAM design, as only a small swing on the bitlines is required. A dynamic latch based sense amplifier is adopted in this design, which distinguishes a small potential difference on two bitlines and locks it to output. The bitline difference required for this amplifier is approximately $1 / 10 V_{D D}$, which is much smaller than an inverter-type sense amplifier. The schematic of the sense amplifier is illustrated in Figure 3.12. 


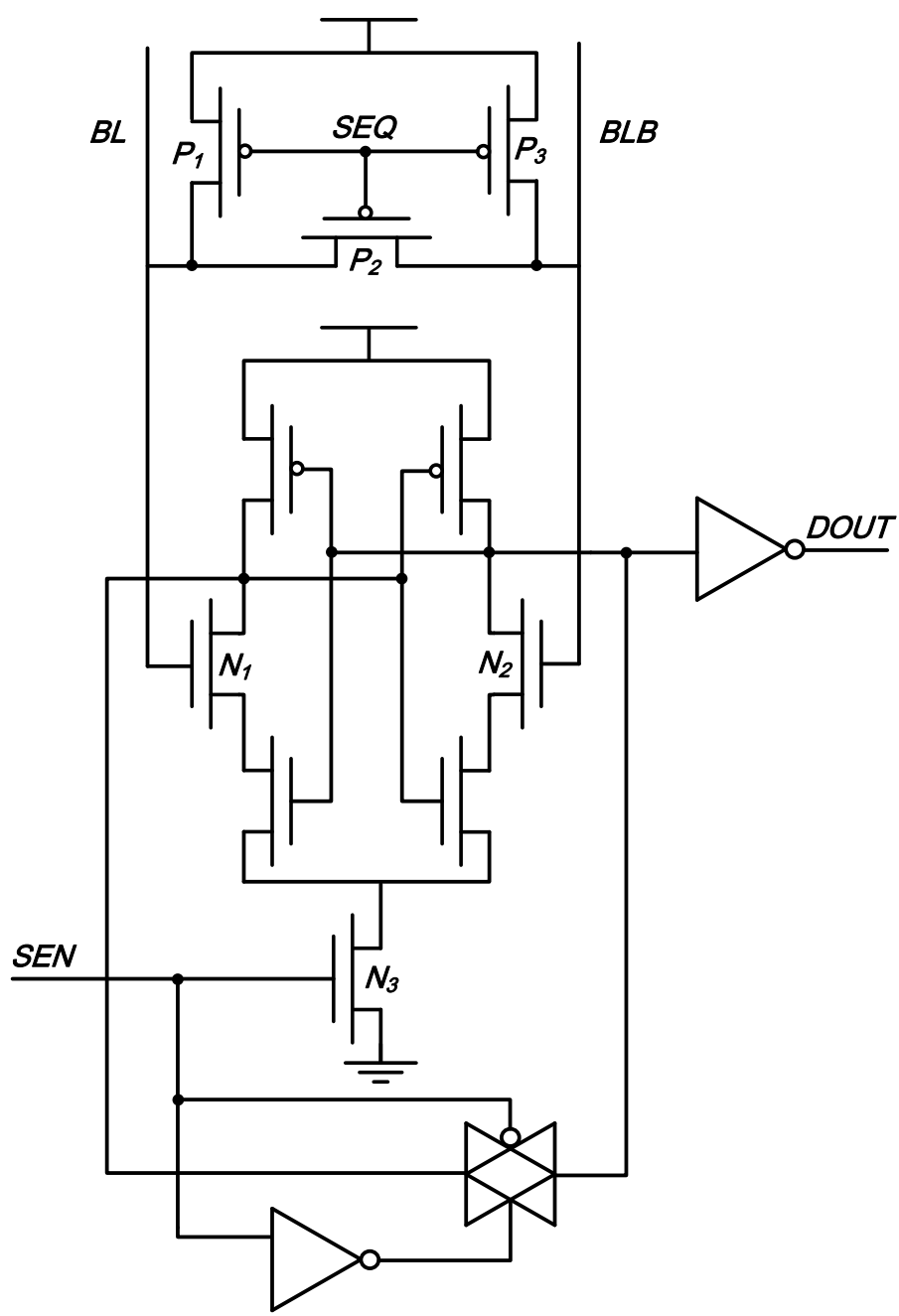

Figure 3.12 Schematic of latch based sense amplifier [40]

This sense amplifier is voltage-sensed for low voltage operations. There are two input transistors $N 1$ and $N 2$ in the cross-coupled inverters of the sense amplifier, each of which is driven by a bitline. When the sense amplifier equalization signal $S E Q$ is low, three p-transistors $(P 1, P 2$ and $P 3)$ equalize $B L$ and $B L B$ and both are pulled up to $V_{D D}$. If sense amplifier enable signal $S E N$ is turned to high, the sense amplifier will start to latch the output according to the bitline's voltage difference.

During the read operation, $S E Q$ and $S E N$ are set to be low first. $B L$ and $B L B$ are pre-charged to the same potential $V_{D D}$. To minimize the potential noises and leakages on $B L$ 
and $B L B$, and also to ensure the equalization process is as quick as possible, these ptransistors must have strong drivability. After equalization, $S E Q$ is set to high to turn off the three pull-up transistors, and the selected memory cell begins to drive $B L$ or $B L B$ low. Following this, SEN is turned high to begin sensing the difference between the two bitlines. When the voltage difference on $B L$ and $B L B$ is large enough to be sensed, which occurs at approximately ten percent of the supply voltage $\left(1 / 10 V_{D D}\right), D O U T$ is locked until the end of this read cycle. In normal SRAM design, SEQ is a short pulse ahead of $S E N$. However, it is complicated to determine the pulse width of $S E Q$, as the SRAM targets on variable $V_{D D}$. In order to make the SRAM more robust and amenable for the SEQ and SEN signal generation circuit design with variable power supply voltages, SEQ and SEN are shorted together rather than using two enable signals. Furthermore, SEQ and SEN are turned to low and high simultaneously.

\subsubsection{Write Circuit}

The write circuit is constructed by two transmission gates, which are controlled by $W W L_{-}$, as demonstrated in Figure 3.13. When $W W L_{-}$is low, both transmission gates are turned off and $B L$ and $B L B$ are isolated with input data. When $W W L_{-}$is high, the transmission gates are turned on, driving $B L$ and $B L B$ to complementary values according to the input data. 


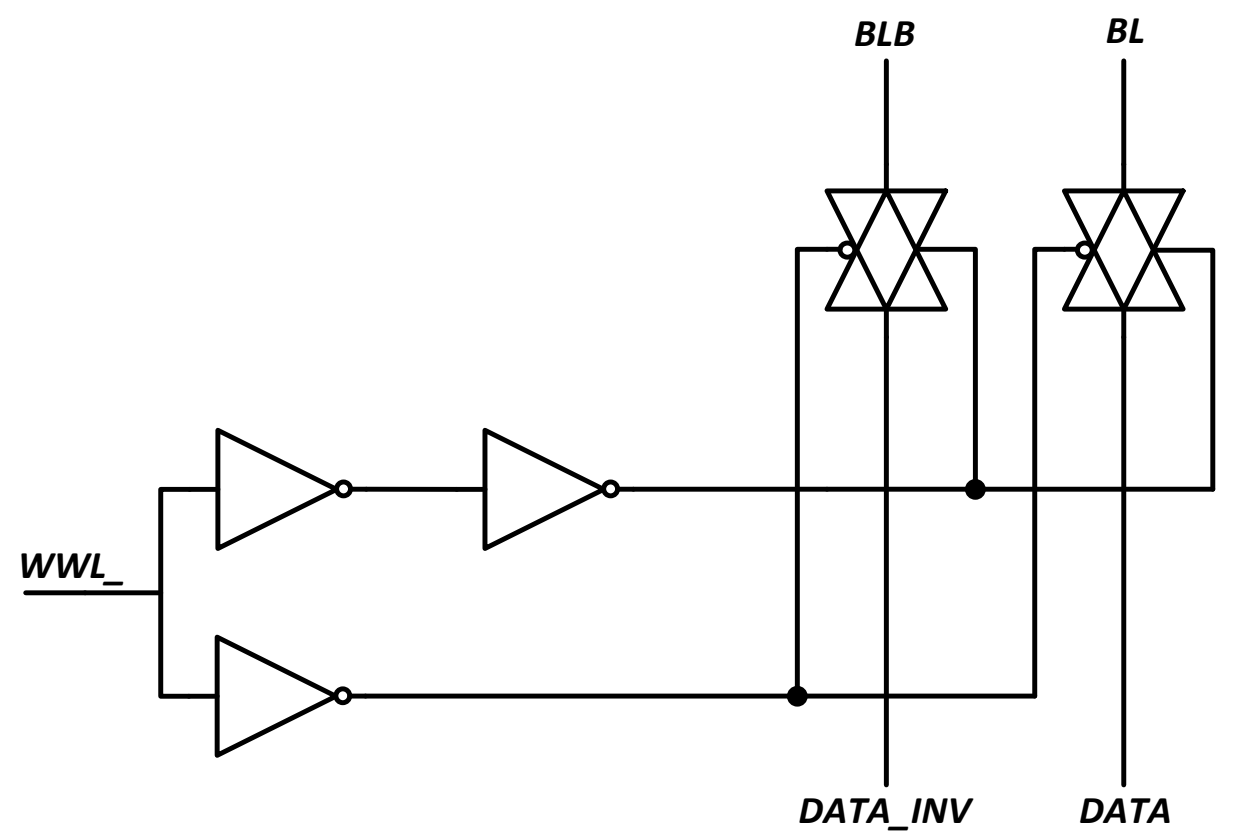

Figure 3.13 Schematic of the write circuit

\subsubsection{Level Shifter}

As the SRAM is target to be radiation tolerant with different power supply voltages, especially for sub-threshold voltages, all inputs and outputs of the SRAM may not be the $1 \mathrm{~V}$ power supply voltage. To facilitate testing, standard digital IOs provided by the component library are required, as it is a complex task to obtain all of the inputs, which are characterized by variable potentials and frequencies, and to efficiently and accurately monitor all of the outputs at a high frequency. However, standard digital IOs shift $2.5 \mathrm{~V}$ to the standard voltage, which is $1 \mathrm{~V}$ for input IOs, and shift standard voltage to $2.5 \mathrm{~V}$ for output IOs. In addition, they are not compatible with the target operating supply voltage, which is $0.3 \mathrm{~V}$ to $1 \mathrm{~V}$. Therefore level shifters are employed to both input and output sides of the SRAM in the chip so that the digital IOs can be used, which is set to $2.5 \mathrm{~V}$ for interfacing with external signals.

For the input signal, $2.5 \mathrm{~V}$ signals being received externally are connected to digital input IOs, which have $2.5 \mathrm{~V}$ input and $1 \mathrm{~V}$ output to the core. To shift the $1 \mathrm{~V}$ signal to $V_{D D}$, 
which is the core supply voltage of the SRAM and ranging from $0.3 \mathrm{~V}$ to $1 \mathrm{~V}$, a buffer is adopted as an input level shifter which has $1 \mathrm{~V}$ input and output as the same potential with $V_{D D}$

For the output signals, a simple buffer is not suitable because a buffer will recognize $0.3 \mathrm{~V}$ as $0 \mathrm{~V}$. Therefore, the outputs from SRAM cannot be translated correctly to output IOs. In order to solve this problem, a sub-threshold level-up shifter is employed, as demonstrated in Figure 3.14 [41]. The level up shifter has two power supplies: $V_{-} l o w\left(V_{D D}: 0.3-1 \mathrm{~V}\right)$ and $V \_h i g h(1 \mathrm{~V})$. Vout will be $V \_h i g h$ if Vin is smaller than half of the $V$ low. Otherwise, Vout will be set to ' 0 '. In this case, Vin from the voltage level of $V \_l o w$ is shifted to a voltage level of $V \_h i g h$, which can be further shifted to $2.5 \mathrm{~V}$ by the digital output IOs. By making use of these level shifters, inputs and outputs of the chip can be easily interfaced with external ICs, such as FPGAs, which will greatly simplify the testing circuits of the SRAM.

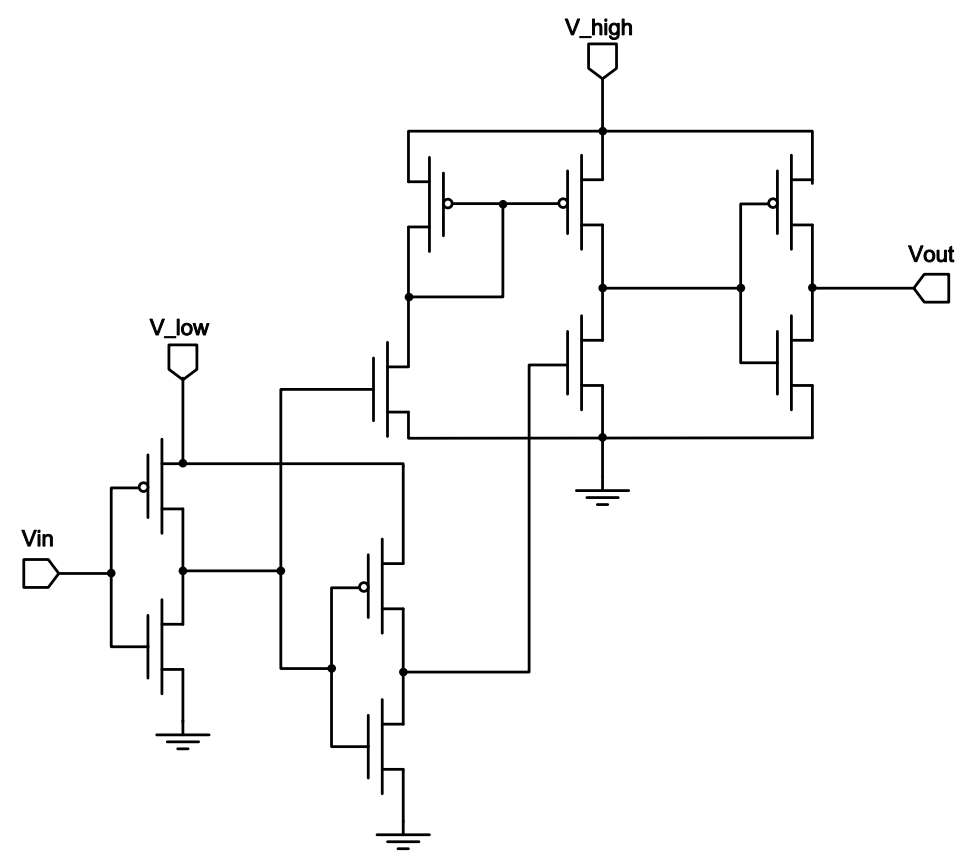

Figure 3.14 Schematic of level-up shifter [41] 


\subsection{Write Operation}

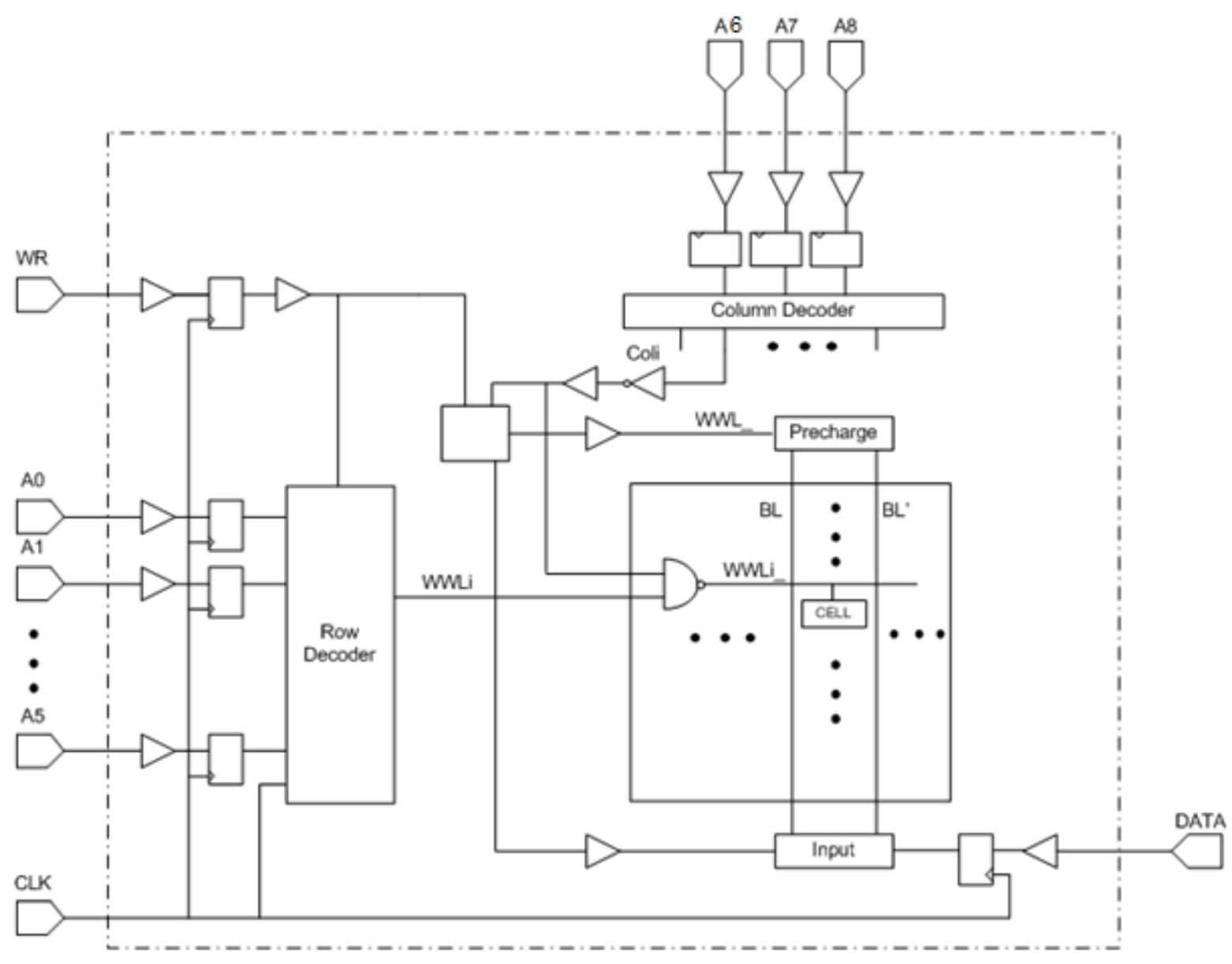

Figure 3.15 SRAM writing diagram

Writing diagram for the SRAM is shown in Figure 3.15. Six row addresses and three column addresses and input data are synchronized by clock first as they enter the chip; The row decoder interprets the six lower addresses $(A O \sim A 5)$ and selects the corresponding write wordline $(W W L)$ of selected row 1, and deselects WWL of all other unselected rows. Column select signals generated by the column decoder enable the local wordline ( $\left.W W L_{-}\right)$of the target column and disable all of the other local wordlines of the unselected columns. The write enable signal is also sent to the write control circuit to enable the transmission-gate to drive complementary data to $B L$ and $B L B$. The timing sequence of a write operation with 
$V_{D D}=1 \mathrm{~V}$ is illustrated in Figure 3.16. It is noted that the writing operation is completed within one clock cycle.

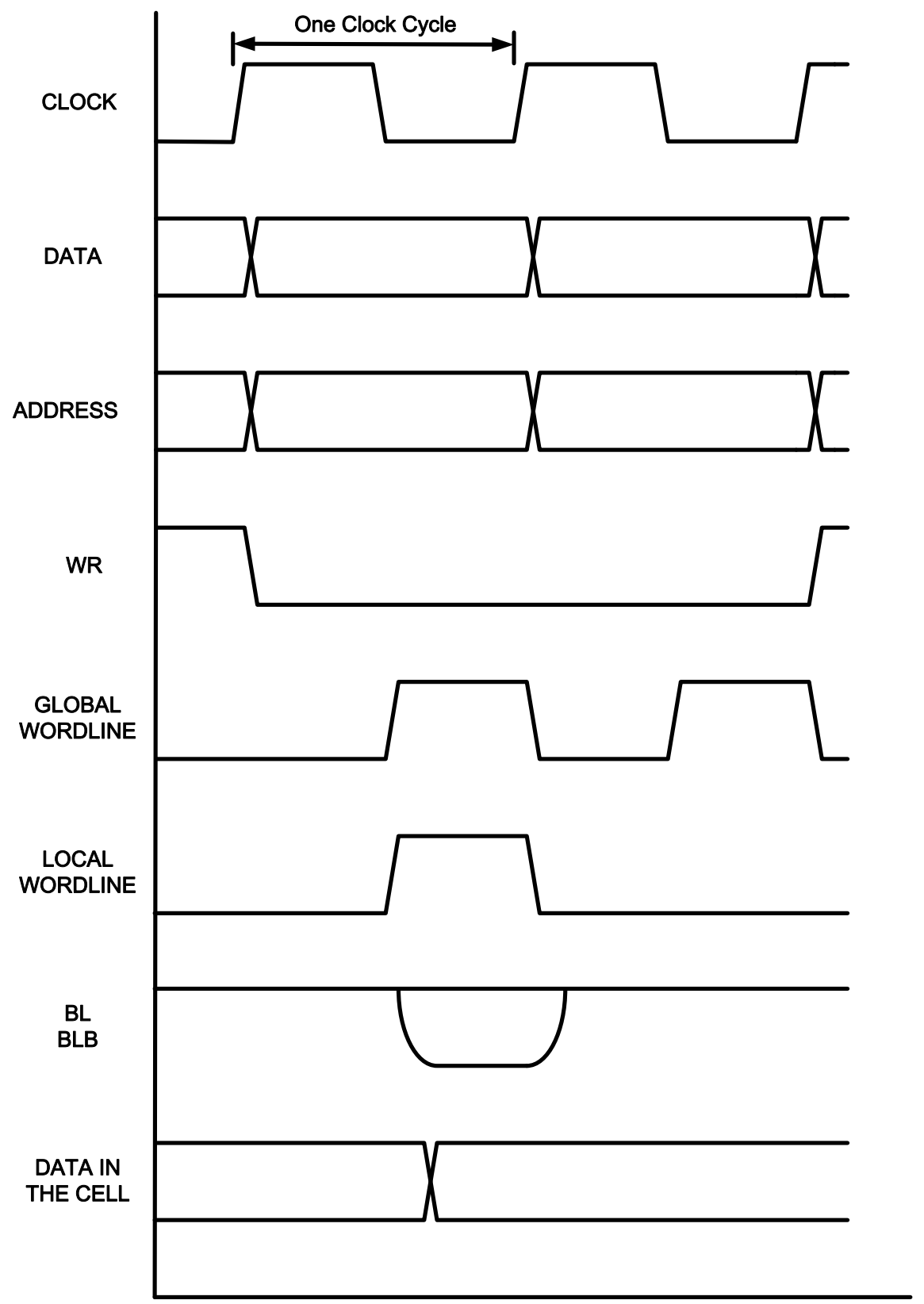

Figure 3.16 Write timing sequence with $V_{D D}=1 \mathrm{~V}$ 


\subsection{Read Operation}

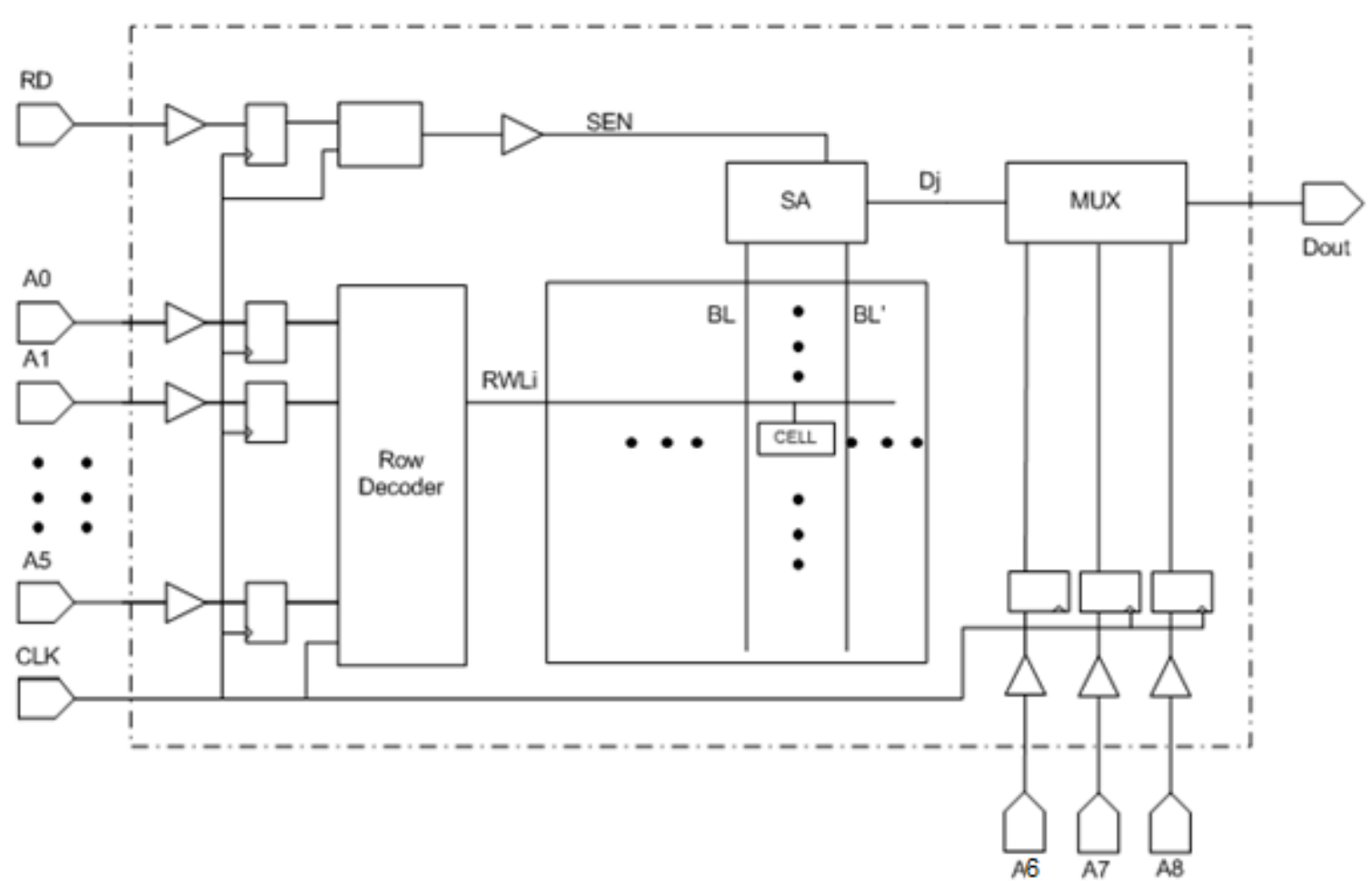

Figure 3.17 SRAM reading diagram

Synonymous with the writing operation, nine addresses are synchronized by the clock first and 64 read wordlines $(R W L)$ are generated by the row decoder with the lower six address bits $(A O \sim A 5)$. For the first half of the clock cycle, the sense amplifier equalize signal $(S E Q)$ is low, and $B L$ and $B L B$ are both pre-charged to a high voltage. For the second half of the clock cycle, $S E Q$ is turned high and $R W L i$ for the row with the target address is set to high, while all the other rows are low. After $R W L i$ is turned high, $B L$ or $B L B$ is pulled low according to the node status in the cell. The sense amplifier enable signal (SEN) is set to high to sense the difference between $B L$ and $B L B$. All eight bytes in the same row, along with the targeted byte, are read out and the outputs of all eight bytes are sent to an eight-to-one multiplexer, which is controlled by the three address bits (A6 A8) to choose the right one. 
The timing sequence of the read operation with $V_{D D}=1 \mathrm{~V}$ and $V_{D D}=0.3 \mathrm{~V}$ is illustrated in Figure 3.18. As evidenced by the timing diagram, the reading operation is also completed in one clock cycle.

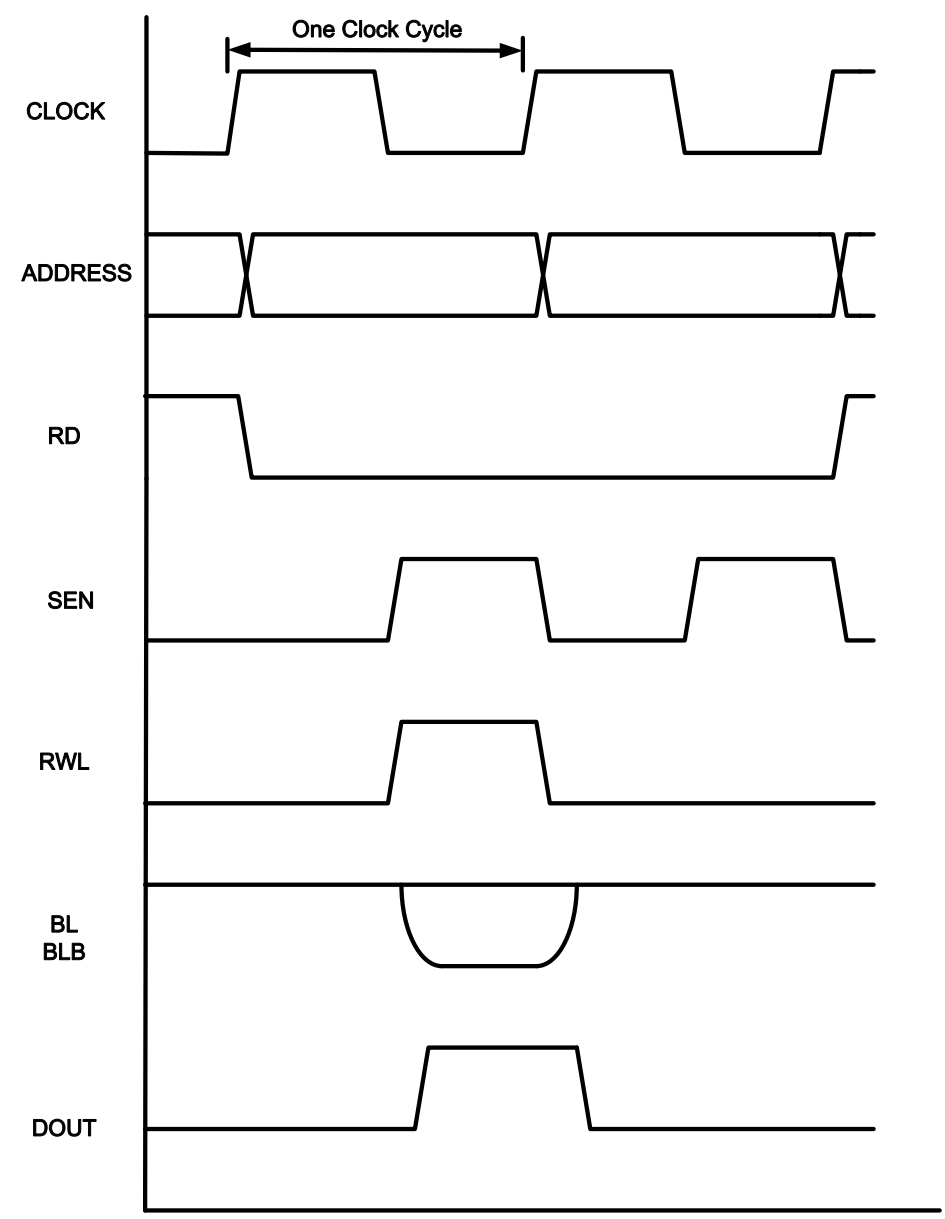

Figure 3.18 Read timing sequence with $V_{D D}=1 \mathrm{~V}$

\subsection{Layout of the SRAM}

A $1.5 \times 1.5 \mathrm{~mm}^{2}$ chip with $16 \mathrm{~K}$ bits SRAM was fabricated in TSMC $65 \mathrm{~nm}$, 9-metal technology. The chip contains four quadrants of SRAM with one kind of memory cell each. The size of the memory cell and peripheral circuits are all the same for comparison purposes. The size of one memory cell is $3.08 \times 2.4 \mathrm{um}^{2}$, and the size of one quadrant of SRAM is $250 \times 180 \mathrm{um}^{2}$, as depicted in Figure 3.19. 


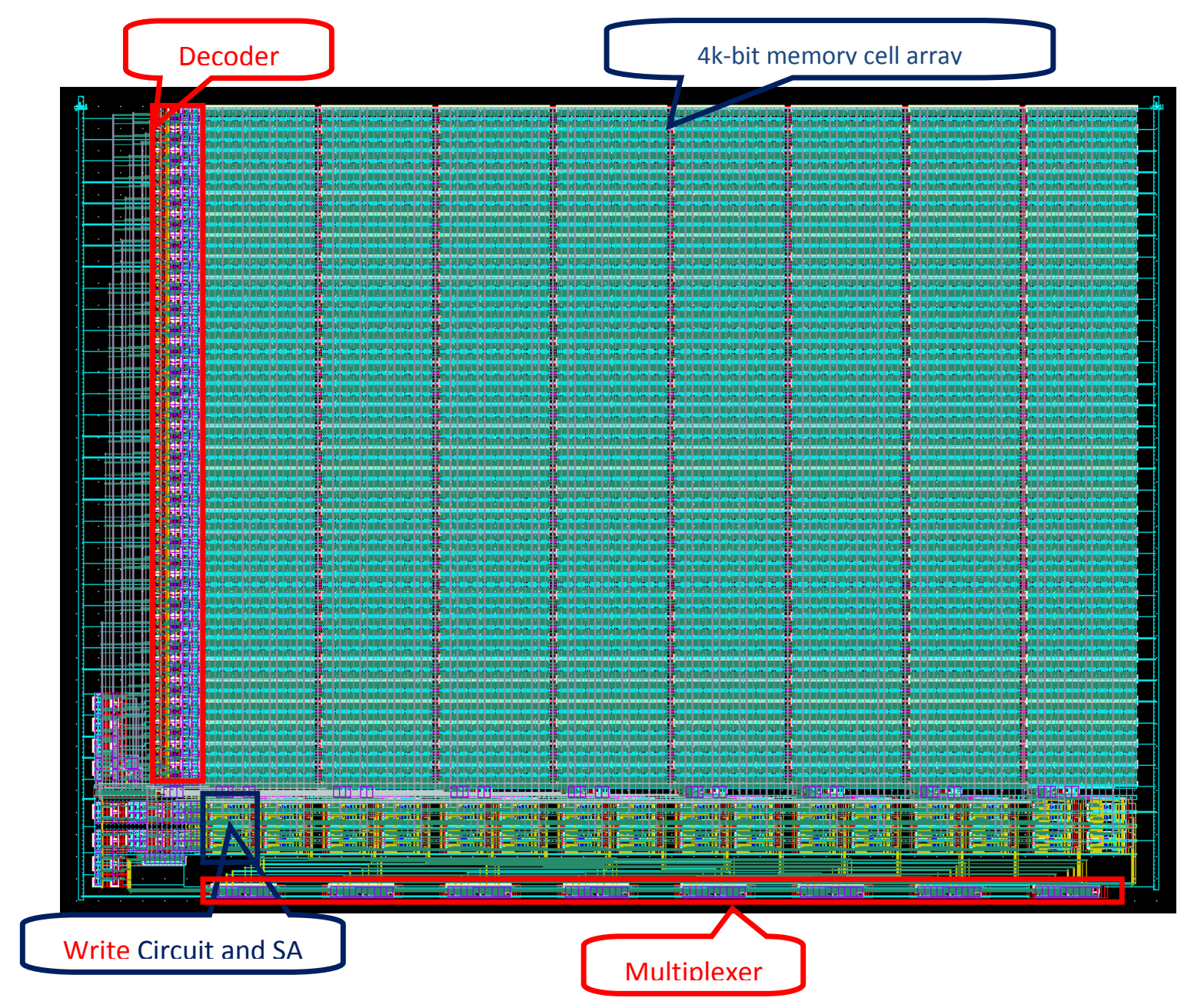

Figure 3.19 Layout of one quadrant of the SRAM design

Several small regions inside of the SRAM layout are not covered by dummy filling metals; these areas can be tested by the pulse laser facility in Saskatchewan Structural 
Sciences Centre (SSSC). The layout for the whole chip and the unfilled regions are illustrated in Figure 3.20.

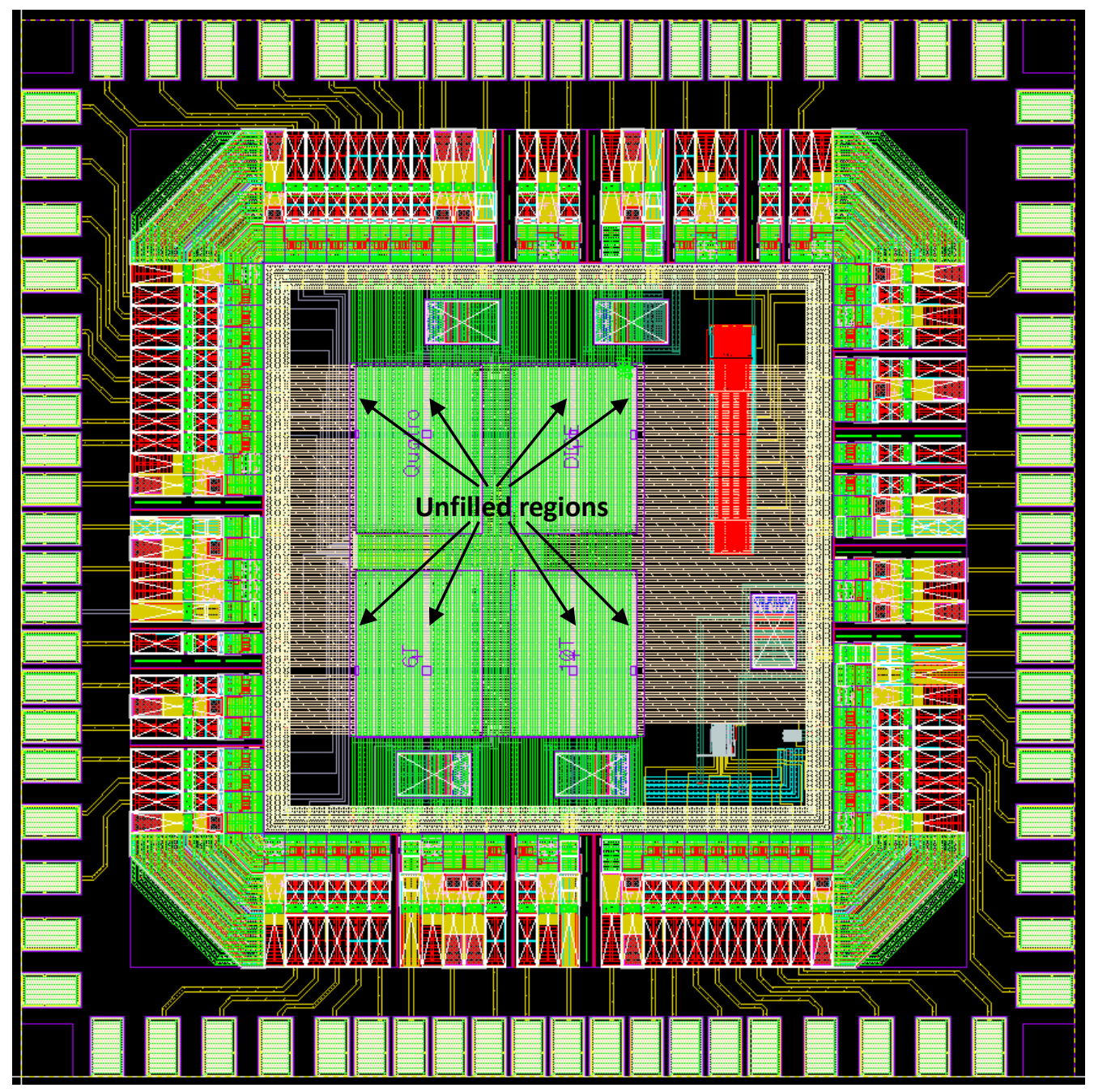

Figure 3.20 Whole chip layout view

Considering the fact that the SRAM is target on the sub-threshold operation, both digital and analog power supply IOs cells from the standard component library are used in the chip. Digital IOs are used for all inputs and outputs, while the analog IOs are used to supply the core supply voltage of the SRAM, ranging from $1 \mathrm{~V}$ to sub-threshold level. The IOs used in the chip design are listed in Table 3.2. 
Table 3.2 IO list for the SRAM chip

\begin{tabular}{|c|c|c|c|c|c|}
\hline IO Name & Function & $\begin{array}{l}\text { Input } \\
\text { Voltage }\end{array}$ & $\begin{array}{l}\text { Output } \\
\text { Voltage }\end{array}$ & $\begin{array}{l}\text { PIN name in SRAM } \\
\text { design }\end{array}$ & No. \\
\hline VDDD_IO & $\begin{array}{l}\text { Power for digital IO ring } \\
\text { ESD protection }\end{array}$ & $2.5 \mathrm{~V}$ & $2.5 \mathrm{~V}$ & N/A & 2 \\
\hline VSSD_IO & $\begin{array}{l}\text { Ground for digital IO ring } \\
\text { ESD protection }\end{array}$ & 0 & 0 & N/A & 2 \\
\hline VDDA_IO & $\begin{array}{l}\text { Power for analog IO ring } \\
\text { ESD protection }\end{array}$ & $2.5 \mathrm{~V}$ & $2.5 \mathrm{~V}$ & N/A & 2 \\
\hline VSSA_IO & $\begin{array}{l}\text { Ground for analog IO ring } \\
\text { ESD protection }\end{array}$ & 0 & 0 & N/A & 2 \\
\hline VDDD_Core & $\begin{array}{l}\text { Power for digital core } \\
\text { ESD protection }\end{array}$ & $1 \mathrm{~V}$ & $1 \mathrm{~V}$ & V_high & 2 \\
\hline VSSD_Core & $\begin{array}{l}\text { Ground for digital core } \\
\text { ESD protection }\end{array}$ & 0 & 0 & GND & 2 \\
\hline VDDA_Core & $\begin{array}{l}\text { Power for SRAM } \\
\text { ESD protection }\end{array}$ & $0.3-1 \mathrm{~V}$ & $0.3-1 \mathrm{~V}$ & V_low & 2 \\
\hline VSSA_Core & $\begin{array}{l}\text { Ground for SRAM } \\
\text { ESD protection }\end{array}$ & 0 & 0 & GND & 2 \\
\hline PDIDGZ & input & $2.5 \mathrm{~V}$ & $1 \mathrm{~V}$ & $\begin{array}{l}\text { DATA0-DATA7, CLK, } \\
\text { WR, RD, A0-A10 }\end{array}$ & 22 \\
\hline PDO12CDG & output & $1 \mathrm{~V}$ & $2.5 \mathrm{~V}$ & DOUT0-DOUT7 & 8 \\
\hline
\end{tabular}




\section{CHAPTER 4 \\ SIMULATION RESULTS}

\subsection{Memory cell simulation}

\subsubsection{Signal Noise Margin}

In order to achieve the objective of operating in sub-threshold voltage range, signal noise margin discussed in Chapter 2 must be taken into account, as it is one of the most important criterions for ultra low-power SRAM design. Signal noise margin can be acquired by voltage transfer characteristic (VTC).

The configuration for $S N M$ simulation is the same as what was depicted in Figure 2.6 and Figure 2.7. In regards to the read noise margin, $B L, B L B$ and $W L$ are all set to be high. For the hold noise margin, $B L$ and $B L B$ are also set to high and $W L$ is biased to $G N D$. The read and hold noise margins can be extracted by VTC curve, which is acquired by sweeping one node of memory cell ( $Q$ or $Q B)$ from 0 to $V_{D D}$ and monitoring the potential on the other node $(Q B$ or $Q)$. Butterfly curves for $R S N M$, as shown in Figure 4.1, for all four cells are obtained by plotting $Q$ versus $Q B$ and, subsequently, plotting $Q B$ versus $Q$. SNM is determined by the size of the maximum square in the butterfly curve. All VTC curves of four

SRAM cell are simulated with $V_{D D}$ changes from $0.3 \mathrm{~V}$ to $1 \mathrm{~V}$ by $0.1 \mathrm{~V}$ step. HSNM and $R S N M$ of the four cells are depicted in Figure 4.2. 

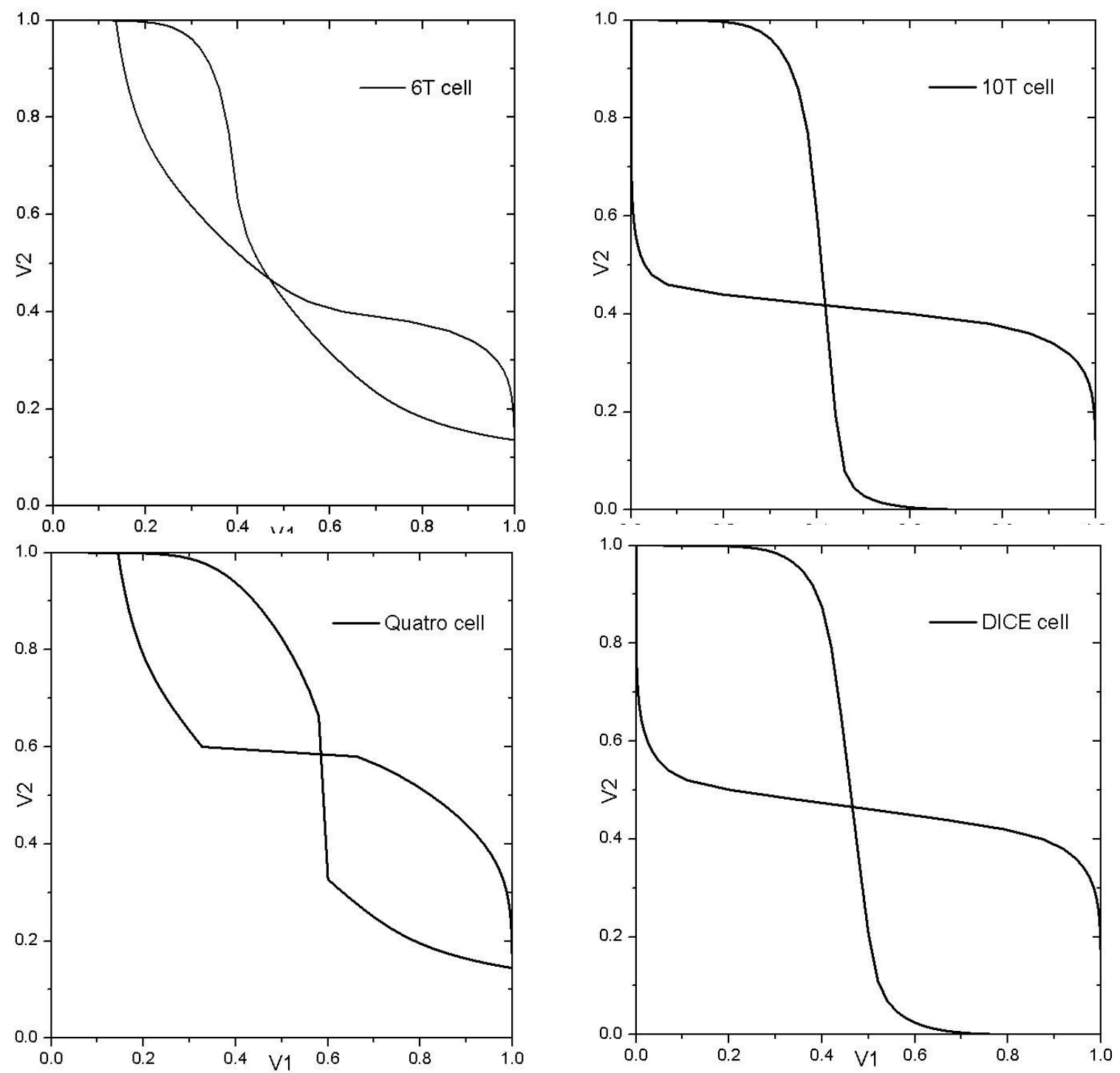

Figure 4.1 Read butterfly curves of the four SRAM cells

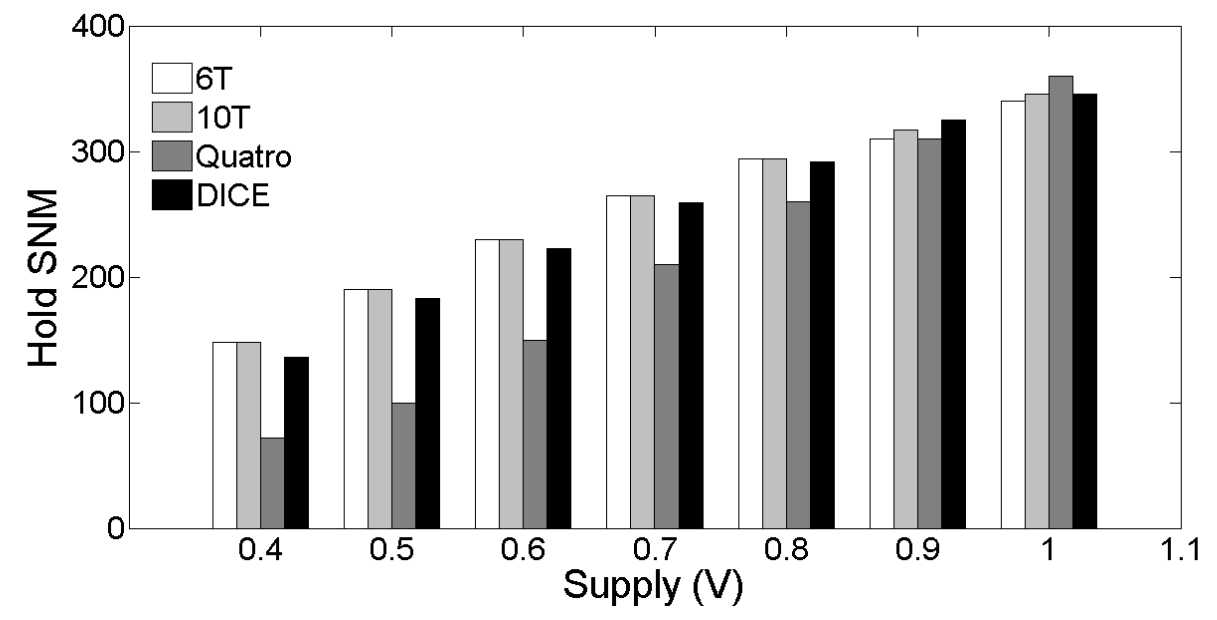




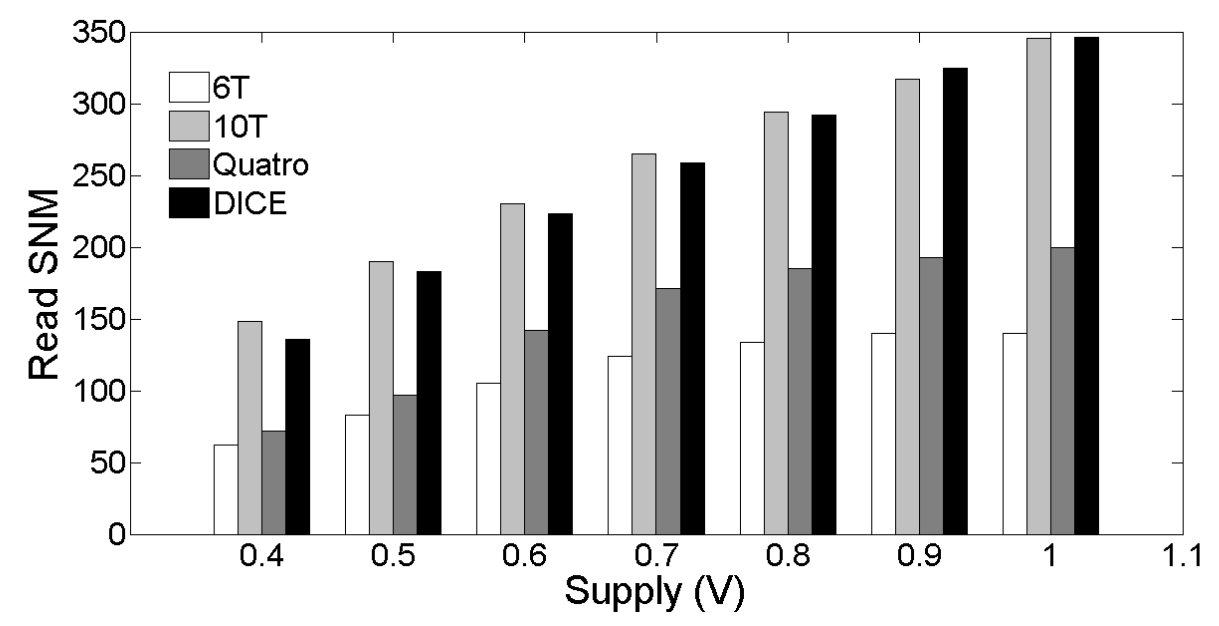

Figure 4.2 Hold and read $S N M$ of four cells from $0.3 \mathrm{~V}$ to $1 \mathrm{~V}$

As shown in the diagrams, the 10T cell and DICE cell have the same RSNM as HSNM. In reference to the Quatro and conventional 6T cell, the RSNM is significantly smaller than HSNM. As the supply voltage decreases to sub-threshold region, the RSNM for the conventional $6 \mathrm{~T}$ cell is reduced to only $42 \mathrm{mV}$, as compared to $101 \mathrm{mV}$ for the $10 \mathrm{~T}$ cell.

\subsubsection{Read and write simulations}

Read and write simulations were performed for the four memory cells to ensure all cells were functional within the proposed supply voltage from $0.3 \mathrm{~V}$ to $1 \mathrm{~V}$. Simulation results for the read and write processes with $V_{D D}=1 \mathrm{~V}$ and $0.3 \mathrm{~V}$ are outlined in Figure 4.3 and Figure 4.4, respectively. 

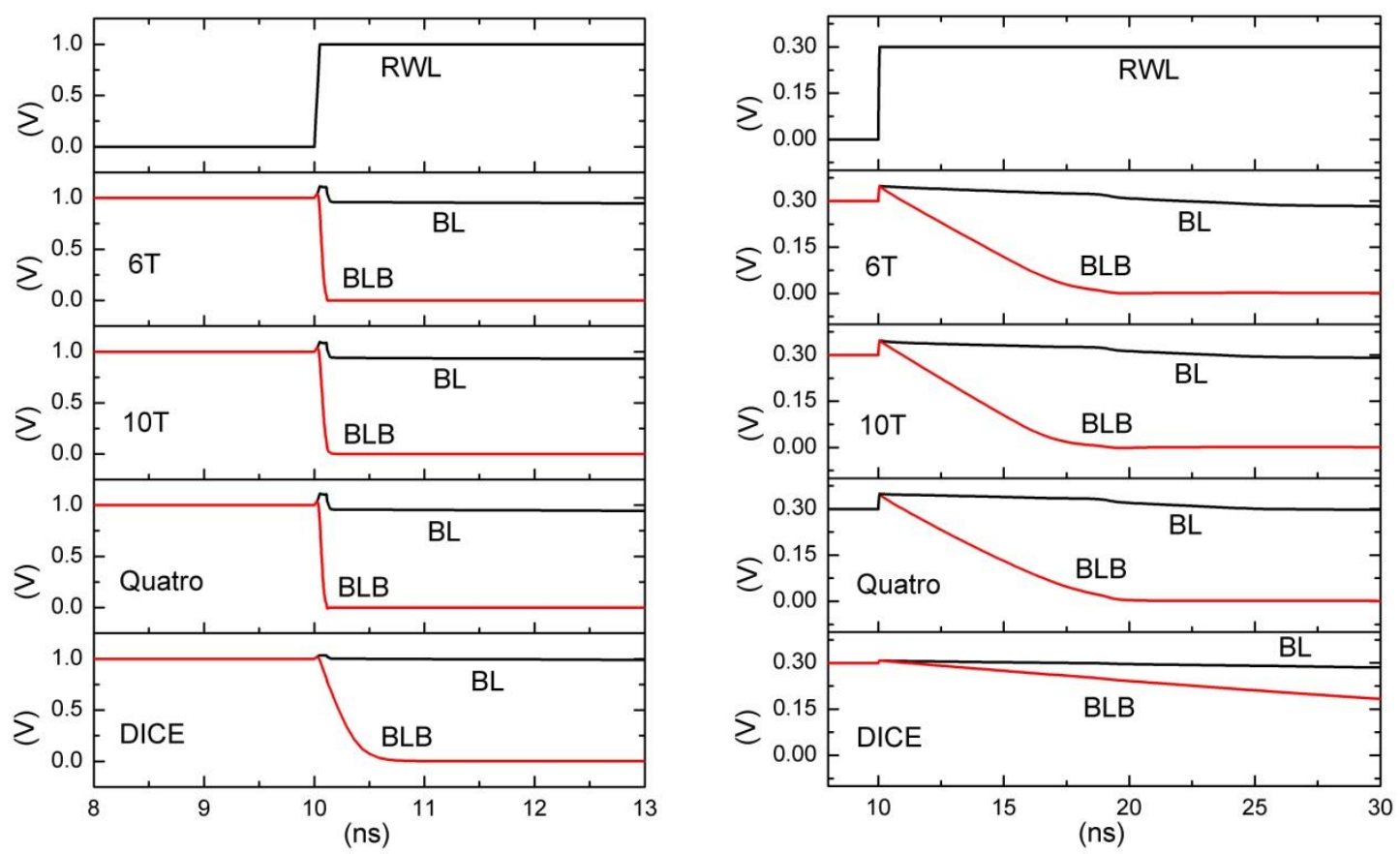

Figure 4.3 Simulation results of $B L, B L B$ and $R W L$ during read cycle with $V_{D D}=1 \mathrm{~V}$ and 0.3 $\mathrm{V}$
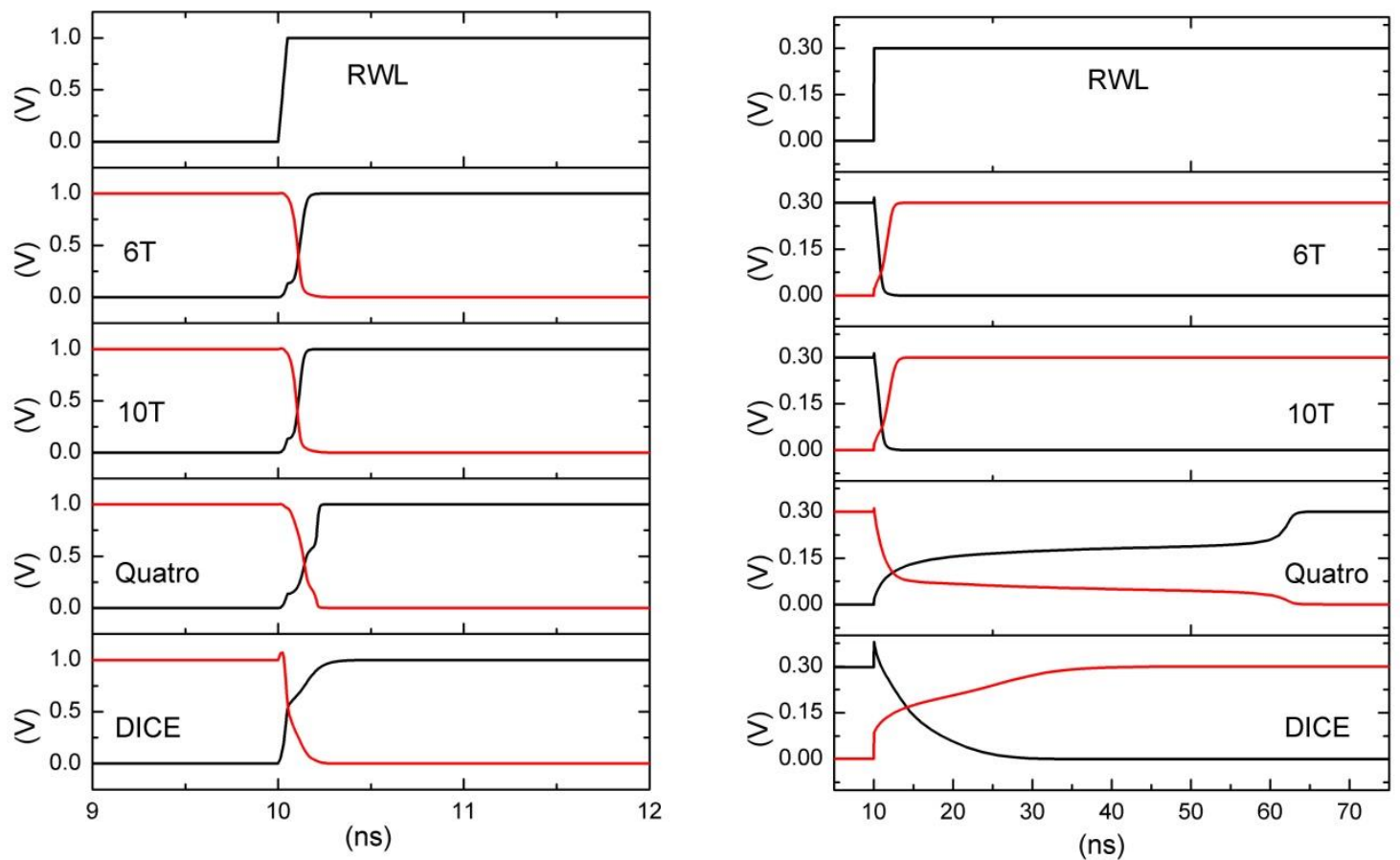

Figure 4.4 Simulation results of storage nodes and $R W L$ during read cycle with $V_{D D}=1 \mathrm{~V}$ and $0.3 \mathrm{~V}$ 
As depicted in the simulation results, both read and write operations are functional with super-threshold and sub-threshold power supply voltages. Both the read time and write time of the four cells are much slower with $V_{D D}=0.3 \mathrm{~V}$, as compared to those of $V_{D D}=1 \mathrm{~V}$. For the read operation, the DICE cell has the slowest read time, while the Quatro cell takes longest amount of time to complete the write operation.

\subsubsection{Critical Charge}

In regards to SRAM, critical charge is defined as the minimum single-event deposited charge required to trigger the upset in SRAM cells. For SRAMs fabricated with nanometer processes, the decreased supply voltage would not only lead to smaller noise margins, but also increase their susceptibilities to single-event upsets (SEUs) caused by particle strikes. This degradation of single-event robustness could be explained by analyzing the qualitative definition of critical charge $Q_{c r i t}$ in (4.1) [7], [8], [9].

$$
Q_{\text {crit }}=C_{N} V_{\text {Supply }}+I_{D} T_{F}
$$

In reference to the above formula, $C_{N}$ is the load capacitance of the struck node, $V_{\text {Supply }}$ is the supply voltage, $I_{D}$ is the maximum current provided by the driving transistor, and $T_{F}$ is the cell flipping time [9]. As the supply voltage drops, the nodal charge $C_{N} V_{\text {Supply }}$ decreases, and the conductivities of on-state transistors also become weaker, limiting their abilities to provide a large enough $I_{D}$ to restore the levels of struck nodes. Both of these two factors result in the decrease of $Q_{c r i t}$, indicating that SRAM cells become more sensitive to SEUs with low supply voltages. 
Table 4.1 Critical charge of four cells

\begin{tabular}{|c|c|c|c|c|c|c|c|c|}
\hline $6 \mathrm{~T}$ & \multicolumn{9}{|c|}{ Critical Charge (fC) } \\
\hline$V_{D D}$ & $0.3 \mathrm{~V}$ & $0.4 \mathrm{~V}$ & $0.5 \mathrm{~V}$ & $0.6 \mathrm{~V}$ & $0.7 \mathrm{~V}$ & $0.8 \mathrm{~V}$ & $0.9 \mathrm{~V}$ & $1 \mathrm{~V}$ \\
\hline${ }^{\prime} 1$ ' $\rightarrow$ ' 0 & 4.90 & 7.70 & 12.6 & 16.79 & 21.69 & 27.29 & 33.58 & 40.58 \\
\hline${ }^{\prime} 0$ ' $\rightarrow{ }^{\prime} 1$ & 5.458 & 13.99 & 27.99 & 45.49 & 62.98 & 81.87 & 101.5 & 119 \\
\hline
\end{tabular}

\begin{tabular}{|c|c|c|c|c|c|c|c|c|}
\hline $10 \mathrm{~T}$ & \multicolumn{9}{|c|}{ Critical Charge (fC) } \\
\hline$V_{D D}$ & $0.3 \mathrm{~V}$ & $0.4 \mathrm{~V}$ & $0.5 \mathrm{~V}$ & $0.6 \mathrm{~V}$ & $0.7 \mathrm{~V}$ & $0.8 \mathrm{~V}$ & $0.9 \mathrm{~V}$ & $1 \mathrm{~V}$ \\
\hline$' 1$ ' $\rightarrow{ }^{\prime} 0$ & 5.248 & 9.097 & 13.3 & 18.2 & 23.79 & 29.39 & 34.99 & 43.99 \\
\hline '0' '> '1' & 6.018 & 14 & 29.39 & 47.58 & 65.78 & 85.37 & 105 & 126 \\
\hline
\end{tabular}

\begin{tabular}{|c|c|c|c|c|c|c|c|c|}
\hline DICE & \multicolumn{7}{|c|}{ Critical Charge (fC) } \\
\hline$V_{D D}$ & $0.3 \mathrm{~V}$ & $0.4 \mathrm{~V}$ & $0.5 \mathrm{~V}$ & $0.6 \mathrm{~V}$ & $0.7 \mathrm{~V}$ & $0.8 \mathrm{~V}$ & $0.9 \mathrm{~V}$ & $1 \mathrm{~V}$ \\
\hline$' 1$ ' $\rightarrow{ }^{\prime} 0$ & $\mathrm{NF}$ & $\mathrm{NF}$ & $\mathrm{NF}$ & $\mathrm{NF}$ & $\mathrm{NF}$ & $\mathrm{NF}$ & $\mathrm{NF}$ & $\mathrm{NF}$ \\
\hline$' 0$ ' $\rightarrow{ }^{\prime} 1$ & $\mathrm{NF}$ & $\mathrm{NF}$ & $\mathrm{NF}$ & $\mathrm{NF}$ & $\mathrm{NF}$ & $\mathrm{NF}$ & $\mathrm{NF}$ & $\mathrm{NF}$ \\
\hline
\end{tabular}

\begin{tabular}{|c|c|c|c|c|c|c|c|c|c|}
\hline \multicolumn{2}{|c|}{ Quatro } & \multicolumn{8}{|c|}{ Critical Charge (fC) } \\
\hline & $V_{D D}$ & $0.3 \mathrm{~V}$ & $0.4 \mathrm{~V}$ & $0.5 \mathrm{~V}$ & $0.6 \mathrm{~V}$ & $0.7 \mathrm{~V}$ & $0.8 \mathrm{~V}$ & $0.9 \mathrm{~V}$ & $1 \mathrm{~V}$ \\
\hline \multirow{2}{*}{ A } & ' 1 ' $\rightarrow>$ ' 0 ' & $\mathrm{NF}$ & $\mathrm{NF}$ & $\mathrm{NF}$ & $\mathrm{NF}$ & $\mathrm{NF}$ & $\mathrm{NF}$ & $\mathrm{NF}$ & $\mathrm{NF}$ \\
\hline & '0’ $\rightarrow$ ' 1 ' & 5.038 & 10.5 & 22.39 & 38.48 & 57.38 & 76.97 & 97.95 & 119 \\
\hline \multirow{2}{*}{$\mathrm{C}$} & ' 1 ' $\rightarrow$ ' 0 ' & 23.1 & 41.99 & 91 & 140 & 196 & 258.9 & 335.9 & 412.9 \\
\hline & '0’ $\rightarrow$ ' 1 ' & $\mathrm{NF}$ & $\mathrm{NF}$ & $\mathrm{NF}$ & $\mathrm{NF}$ & $\mathrm{NF}$ & $\mathrm{NF}$ & $\mathrm{NF}$ & $\mathrm{NF}$ \\
\hline
\end{tabular}

To imitate the strike induced pulse described in 2.3 .1 , one commonly used model is a current source with exponential rising and falling edge. The current source has a rapidly rising edge ( 10ps) and a gradually falling edge ( 100ps). If the node is flipped from ' 0 ' to ' 1 ', a current pulse flowing from $V_{D D}$ to the node is used. The second condition stipulates that one node is flipped from ' 1 ' to ' 0 ', and in this case, a current pulse flowing from ground to the node is adopted. Simulations were carried out by changing the current pulse amplitude, while keeping the pulse width constant. The injection charge can be calculated by integration of the transient current, as displayed in equation 4.2 . 


$$
Q_{c r i t}=\int_{t 0}^{t f} I_{i n j}(t) d t
$$

The simulation results for the critical charge of four cells are listed in Table 4.1. In the table, NF signifies that no flip occurs.

Conclusions can be drawn from the above simulation results.

1. The conventional $6 \mathrm{~T}$ cell has the lowest critical charge, while the $10 \mathrm{~T}$ cell has a slightly higher critical charge than the 6T cell, and both cells can be easily flipped by radiation when the supply voltage ranges from $0.3 \mathrm{~V}$ to $1 \mathrm{~V}$.

2. SEU cannot be triggered to the DICE cell if only the single node is affected, regardless of how large the injected current is.

3. The Quatro cell can be upset in two cases: one is to flip ' 0 ' to ' 1 ' at node $A$, and the other one is to flip ' 1 ' to ' 0 ' at node $C$. However, the critical charge required for these kinds of flips is much larger than that of the conventional $6 \mathrm{~T}$ cell; the charge is $\sim 3$ times for node $A$ and $\sim 10$ times for node $C$. In regards to other conditions, Quatro cells are SEU tolerant.

4. For conventional $6 \mathrm{~T}$ cells, the critical charge required to flip the node from ' 0 ' to ' 1 ' is about 3 times higher than the charge needed to flip the node from ' 1 ' to ' 0 '. As discussed in 2.3.2, the most sensitive node for SEU is the reverse-biased transistors in the substrate. Recall the schematic of conventional $6 \mathrm{~T}$ cell in Figure 2.1; assuming that the initial state is $Q=$ ' 1 ', if $Q$ is flipped from ' 1 ' to ' 0 ', the transistor which is struck by the particle is $N 2$. If $Q B$ is upset from ' 0 ' to ' 1 ', the transistor got struke by the particle is $P 1$. Both $P 1$ and $N 2$ are reverse-biased transistors. As $P 1$ lies in a well while $N 2$ is located in the substrate, less energy is required to flip $N 2$ than $P 1$, according to the theory outlined in 2.3.2. 
5. Critical charge is reduced along with a decrease in supply voltage $V_{D D}$ as discussed in this chapter.

\subsection{Simulation of Sense Amplifier}

As a critical component of SRAM, sense amplifiers must be stable and fast. In order to ensure stability of the sense amplifier in the design, Monte Carlo simulation was carried out 100 times for each supply voltage, from $0.3 \mathrm{~V}$ to $1 \mathrm{~V}$. Simulation results for $V_{D D}=1 \mathrm{~V}$ and $V_{D D}=0.3 \mathrm{~V}$ are depicted in Figure 4.6 and Figure 4.7. In the simulation setup, the initial condition stipulates that both $B L$ and $B L B$ equal $V_{D D}$ and the $S E N$ signal is low. Then at 10.0 ns, $B L$ and $B L B$ are set to be $1 \mathrm{~V}, 900 \mathrm{mV}$ respectively for $V_{D D}=1 \mathrm{~V}$; and for $V_{D D}=0.3 \mathrm{~V}, B L$ and $B L B$ are set to be $300 \mathrm{mV}, 200 \mathrm{mV}$. SEN is set to high at the same time. Outputs from the sense amplifier are plotted 100 times. From the simulation results, it can be concluded that the sense amplifier is stable for both super-threshold and sub-threshold supply voltages. Delay is measured from $0.5 V_{D D}$ of the $S E N$ to $0.5 V_{D D}$ of the output. For $V_{D D}=1 \mathrm{~V}, 99 \%$ of the delay dropped in the 25.3 ps to 32.3 ps region. For $V_{D D}=0.3 \mathrm{~V}, 99 \%$ of the delay was between 3.9 ns to $8.38 \mathrm{~ns}$. 


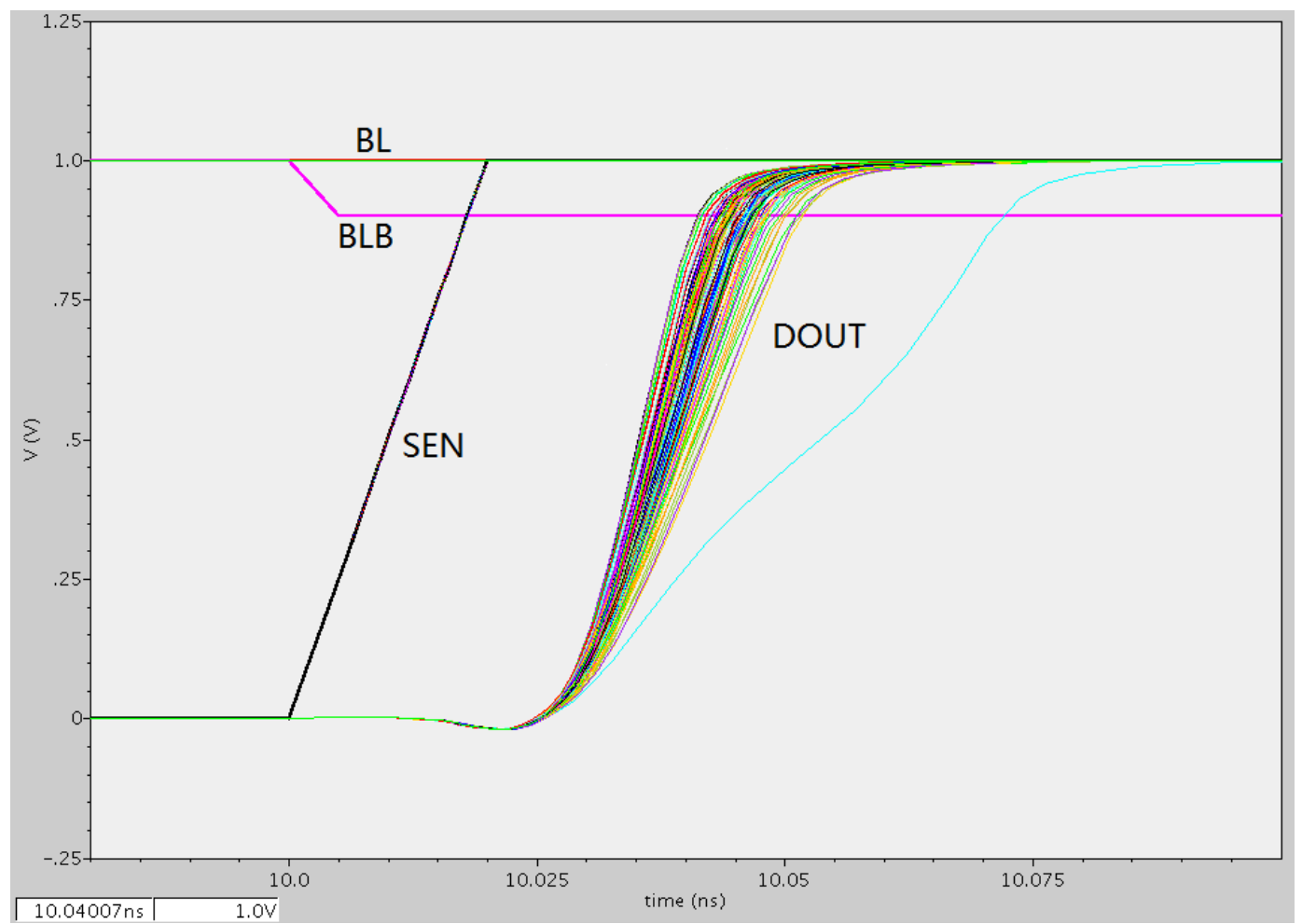

Figure 4.6 Monte Carlo simulation of sense amplifier with $V_{D D}=1 \mathrm{~V}$

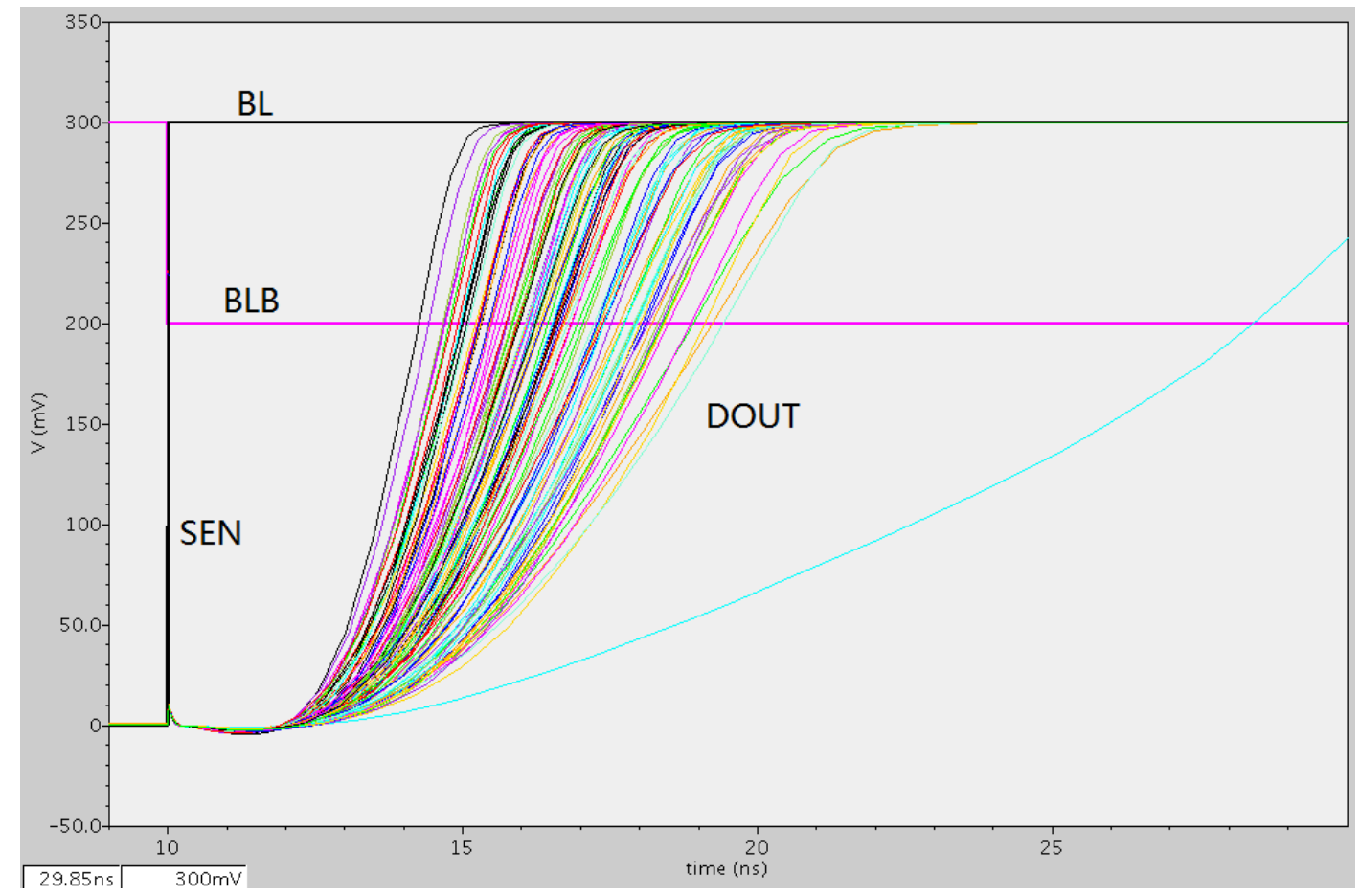

Figure 4.7 Monte Carlo simulation of sense amplifier with $V_{D D}=0.3 \mathrm{~V}$ 


\subsection{Simulation of Level Shifter}

Level up and level down shifters are employed between the IOs and input and output signals of the SRAM, as discussed in the previous chapter. Both level up and level down shifters must be functional for the full supply voltage range (from $0.3 \mathrm{~V}$ to $1 \mathrm{~V}$ ). In regards to the input level down shifter, the input signal is $1 \mathrm{~V}$ and the output signal has the same voltage with the SRAM power supply voltage $V_{D D}$, from $0.3 \mathrm{~V}$ to $1 \mathrm{~V}$. For the output level up shifter, input signals are the outputs from the SRAM, and therefore have the same potential with $V_{D D}$. Correspondingly, the output from the level shifter to IO is $1 \mathrm{~V}$, which is the standard voltage. Simulation results of both level down and level up shifters with the SRAM power supply is equal to $0.3 \mathrm{~V}$ and $0.7 \mathrm{~V}$, as depicted in Figure 4.8 and Figure 4.9, respectively.

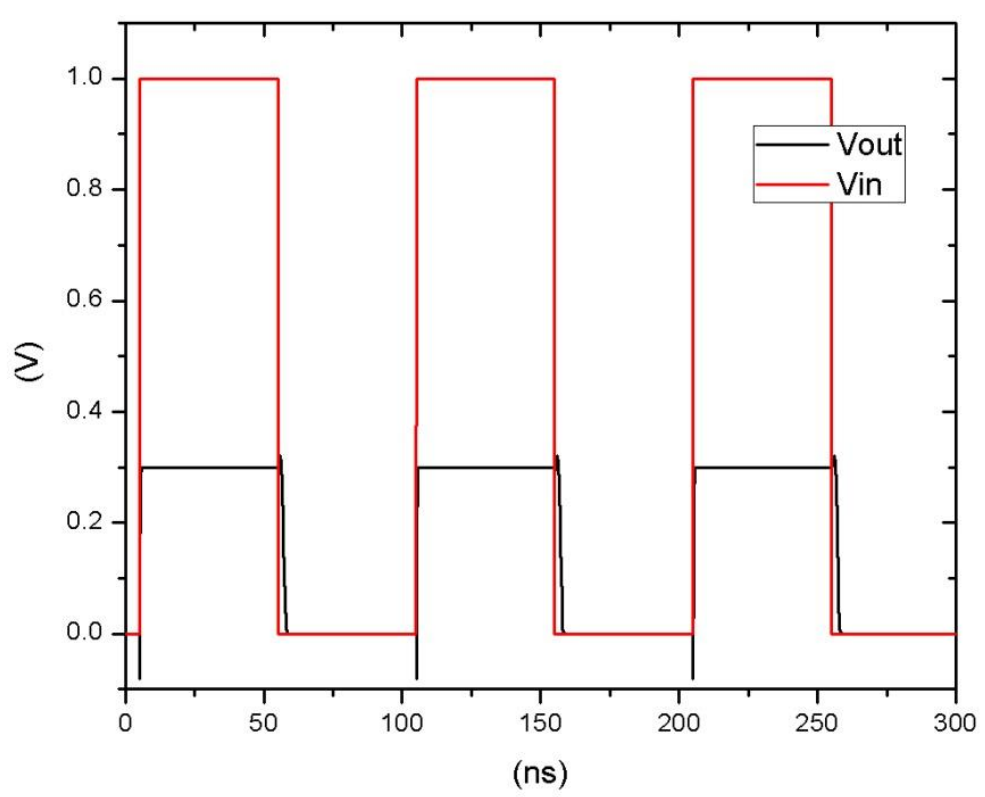




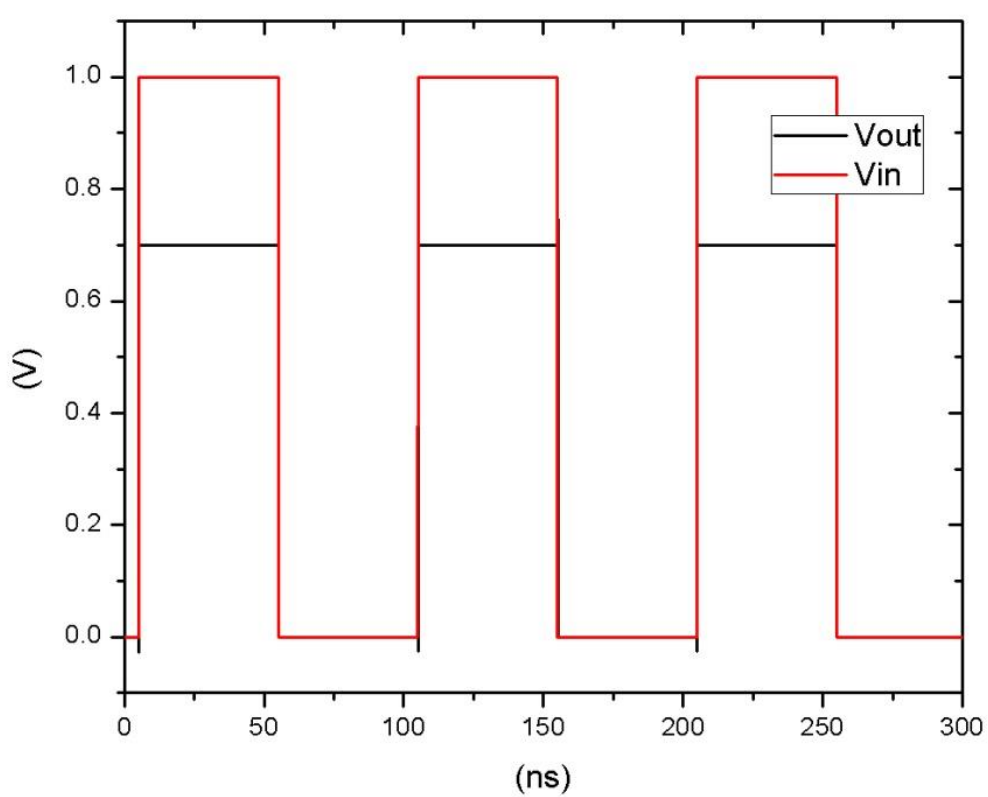

Figure 4.8 Level down shifter with output voltage $0.3 \mathrm{~V}$ and $0.7 \mathrm{~V}$

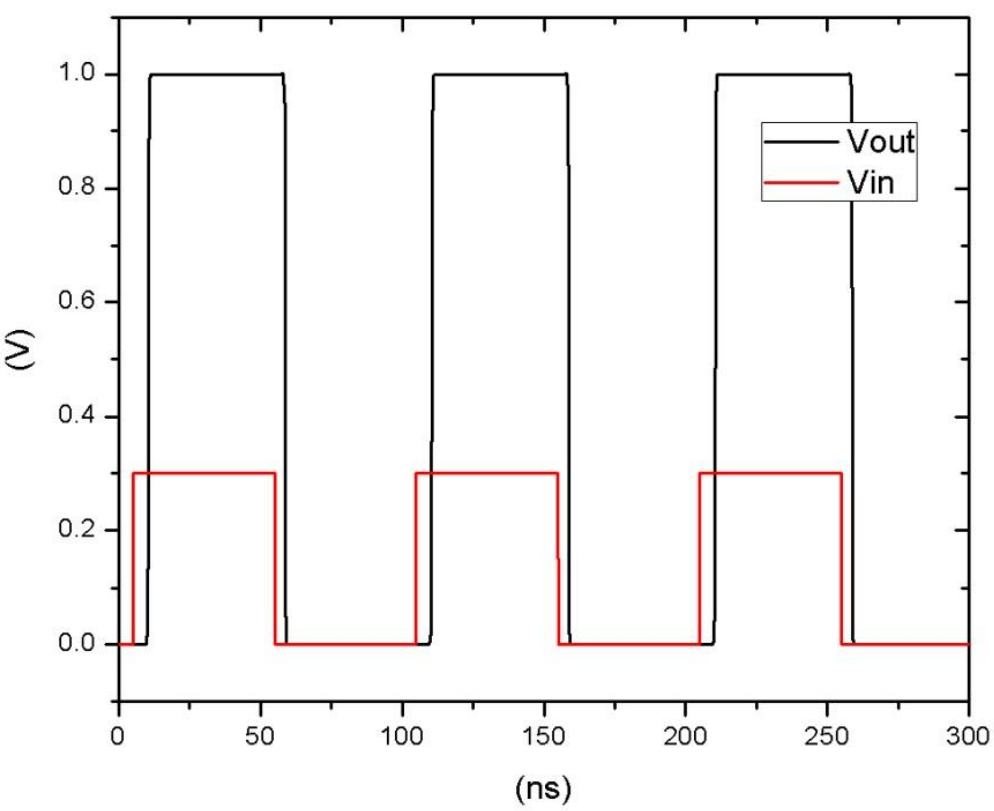




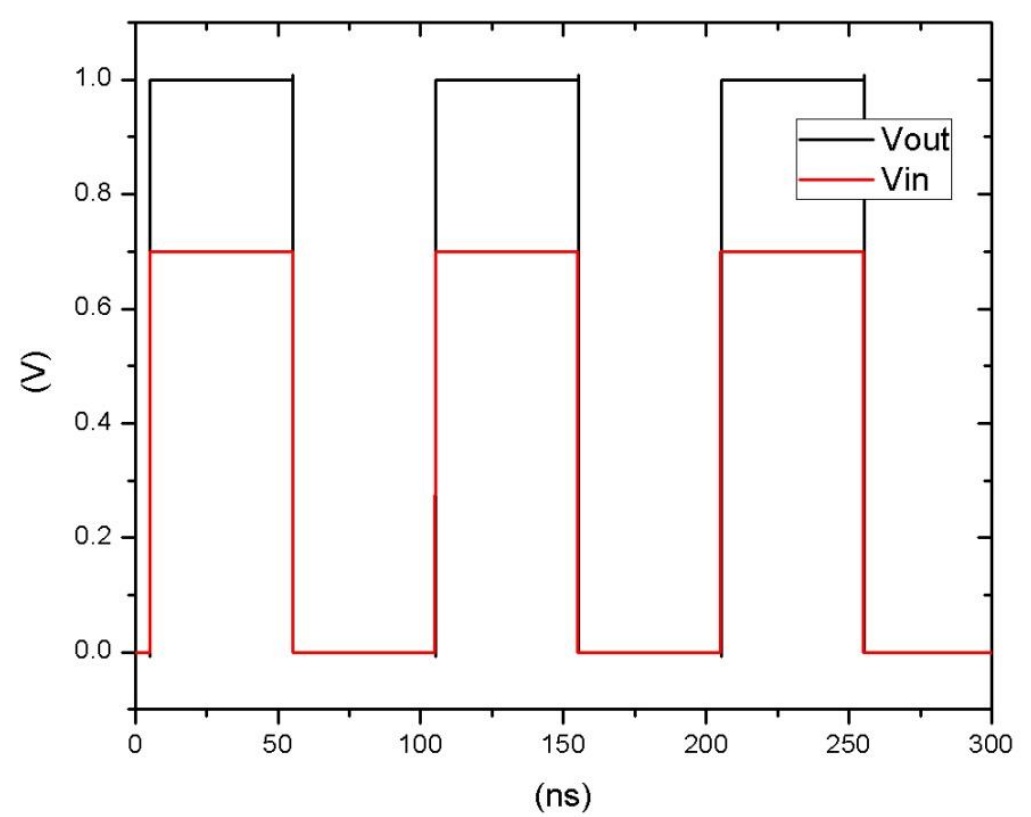

Figure 4.9 Level up shifter with input voltage $0.3 \mathrm{~V}$ and $0.7 \mathrm{~V}$

\subsection{Simulation of Whole SRAM}

In regards to whole chip simulation, it is impossible to verify every address for both the read and write operation, as it takes an impractically long period of time with the use of a single desktop computer. In this work, the lowest two addresses 000000000 and 000000001 are simulated. Data '10101010' and '01010101' are written to the first address 000000000 and second address 000000001 , respectively, and then read from the two addresses to verify the validity of the reading and writing operations. In the simulation, a $5 \mathrm{MHz}$ clock frequency is employed as the unified frequency for all supply voltages. Simulations are carried out for the four SRAM arrays with a supply voltage from $0.3 \mathrm{~V}$ to $1 \mathrm{~V}$. In this thesis, selected simulation results for 6T SRAM with $V_{D D}=0.3 \mathrm{~V}, 0.7 \mathrm{~V}$ and $1 \mathrm{~V}$ are illustrated in Figure 4.10, Figure 4.11 and Figure 4.12. Signals coming to and from IOs, as well as some important internal signals in Figure 2.15 and Figure 2.16, are also shown in the figures. As depicted in 
the simulation results, it is apparent that the memory cells and all the peripheral circuits work as designed in Chapter 3. In the waveforms, the $1 \mathrm{~V}$ signals for digital IOs are red, and named XX_IN for inputs and XX_1V for outputs.

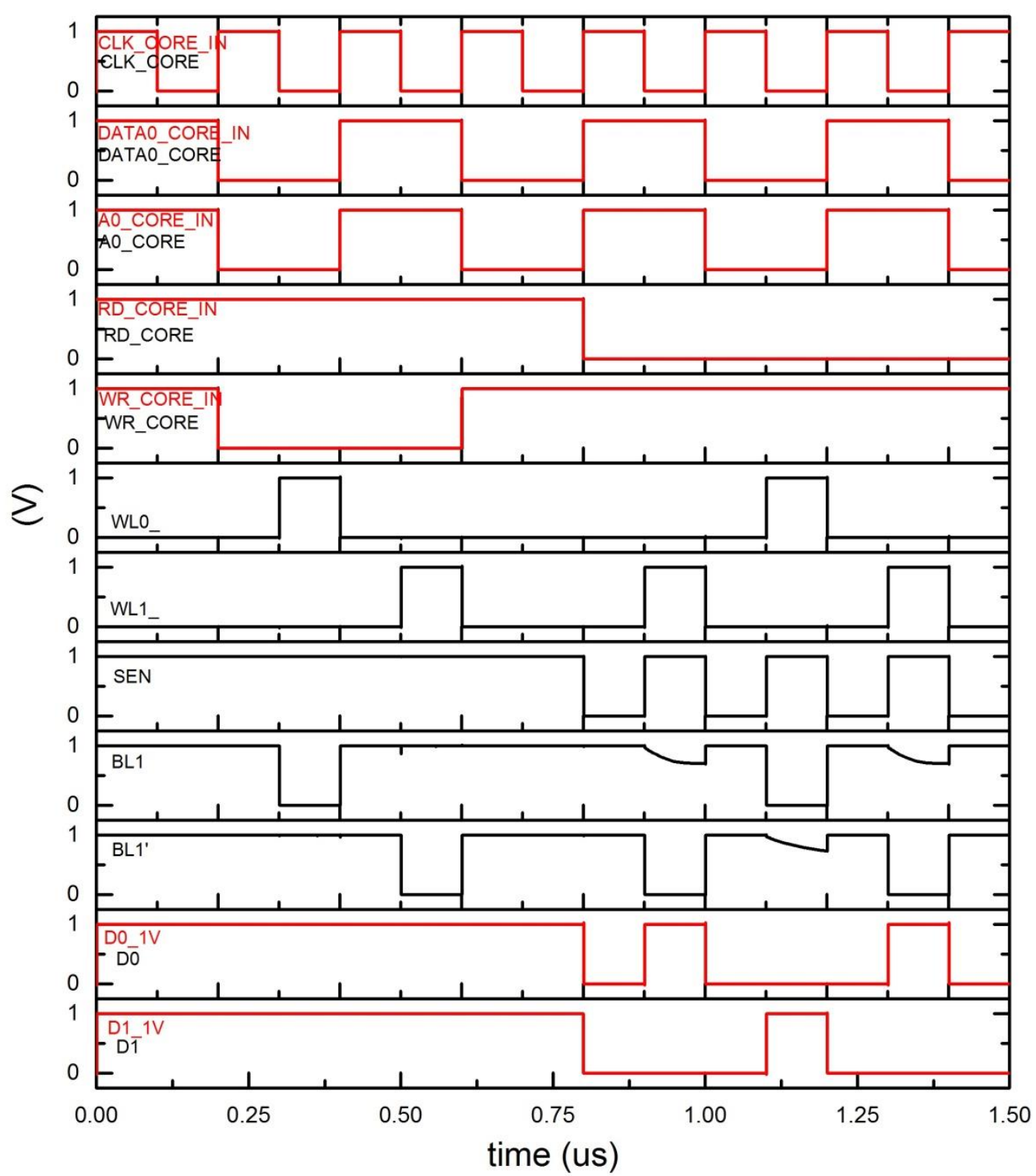

Figure 4.10 Whole 6T SRAM with $V_{D D}=1 \mathrm{~V}$ 


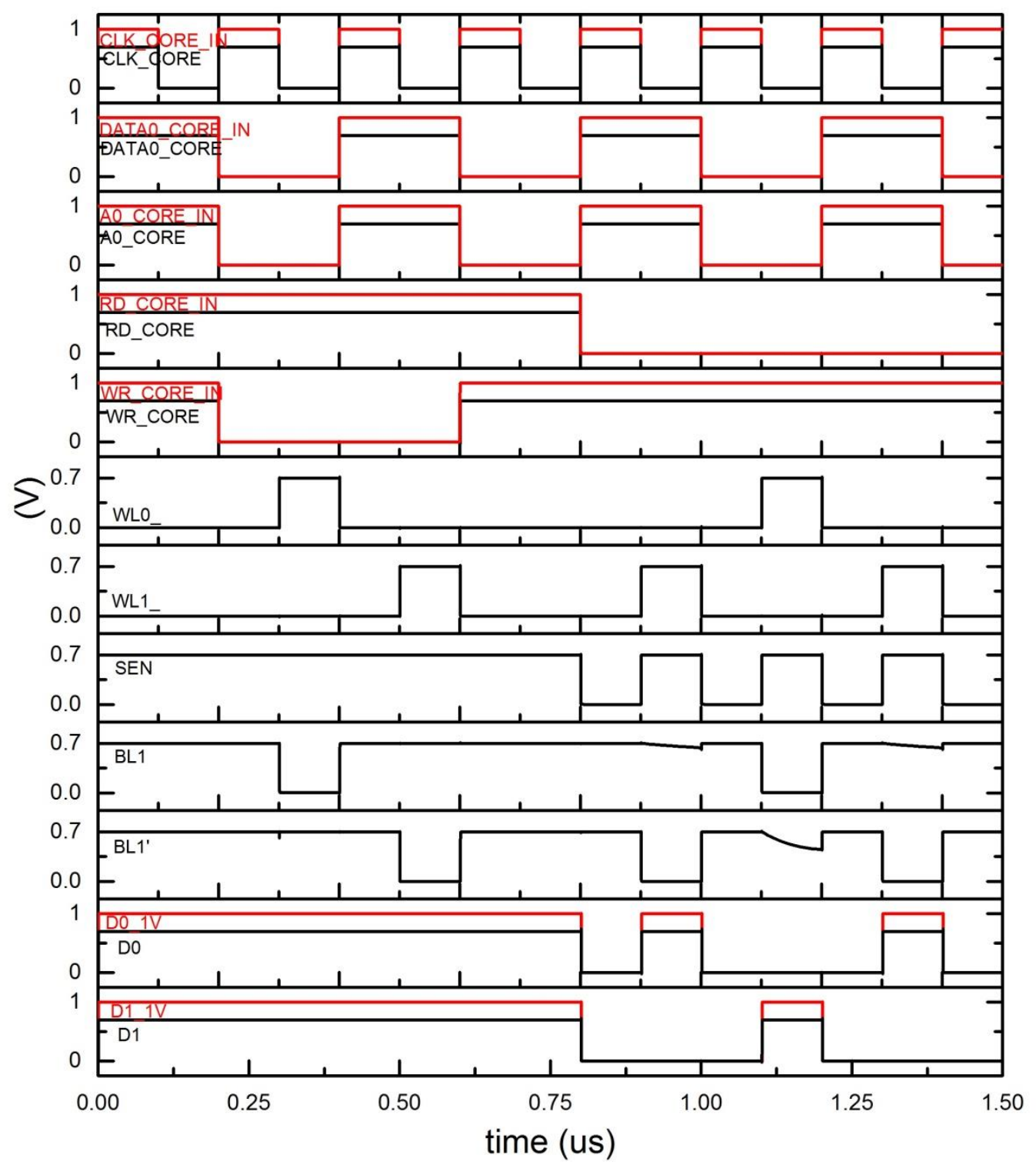

Figure 4.11 Whole 6T SRAM with $V_{D D}=0.7 \mathrm{~V}$ 


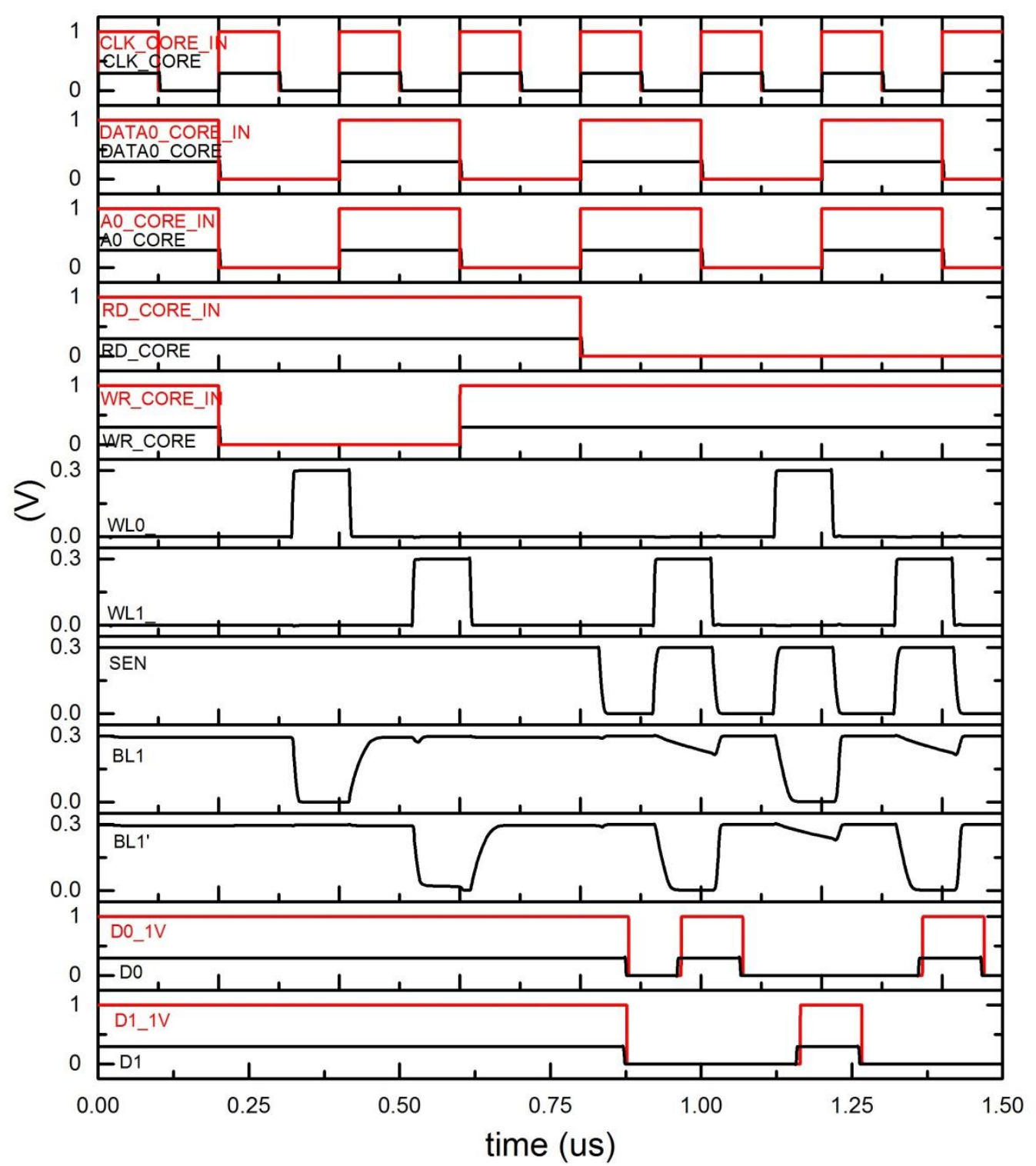

Figure 4.10 Whole 6T SRAM with $V_{D D}=0.3 \mathrm{~V}$ 


\section{CHAPTER 5}

TEST RESULT

\subsection{Test system setup}

The SRAM design was fabricated in TSMC CMOS 65nm, 9 metal technology. The package for the test chip is 80-pin Surface Mount Ceramic Quad Flat Package (CQFP80). In order to test the chip, a Printed Circuit Board (PCB) was designed and had the SRAM test chip soldered on it. The PCB was connected to a custom-designed FPGA testing system with Dual In-line Memory Module (DIMM). One 22-Ohm resistor was attached to each pin of the SRAM for impedance matching purposes. The PCB is illustrated in Figure 5.1.

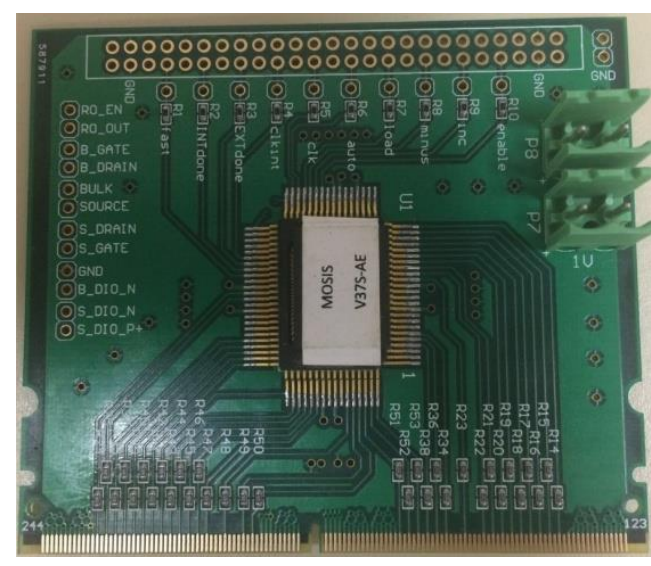

Figure 5.1 Photo of PCB with the SRAM chip soldered

The SRAM to be tested (DUT) is connected to a Virtex 5 FPGA board by inserting the PCB into the DIMM slot on the FPGA board. The test system setup is outlined in Figure 5.2. This block diagram includes the DUT, Virtex 5 FPGA, W7200MCU, internet switch, laptop computer, a regulator board and a power supply. The power regulator board provides the power supplies for the FPGA ( $5 \mathrm{~V}$ and $2.5 \mathrm{~V}$ ), the MCU of the Ethernet controller (3.3 V), and also the SRAM test chip, which includes VDDA_IO (2.5 V), VDDD_IO (2.5 V), and 
VDDD_CORE $(1 \mathrm{~V})$. Considering the fact that the regulator board cannot provide a voltage lower than $1 \mathrm{~V}$, an extra power supply is required to provide the analog power supply (0.3-1 V) for the the SRAM. Besides the regulator board and power supply, W7200MCU and the switch is adopted to allow the FPGA board to communicate with the computer. A program written in $\mathrm{C \#}$ is developed for test configuration and analyzing test results. The program is divided into three parts, the first of which being to load the test configuration and send them to MCU, the second part being to receive outputs sending from MCU and save them in a file, and the last part being to analyze the outputs and compare them with data written into the SRAM and get the error bit map. MCU programmed in $\mathrm{C}$ is used to transfer the test configuration from computer to FPGA, as well as to transfer output data read from the SRAM to the computer.

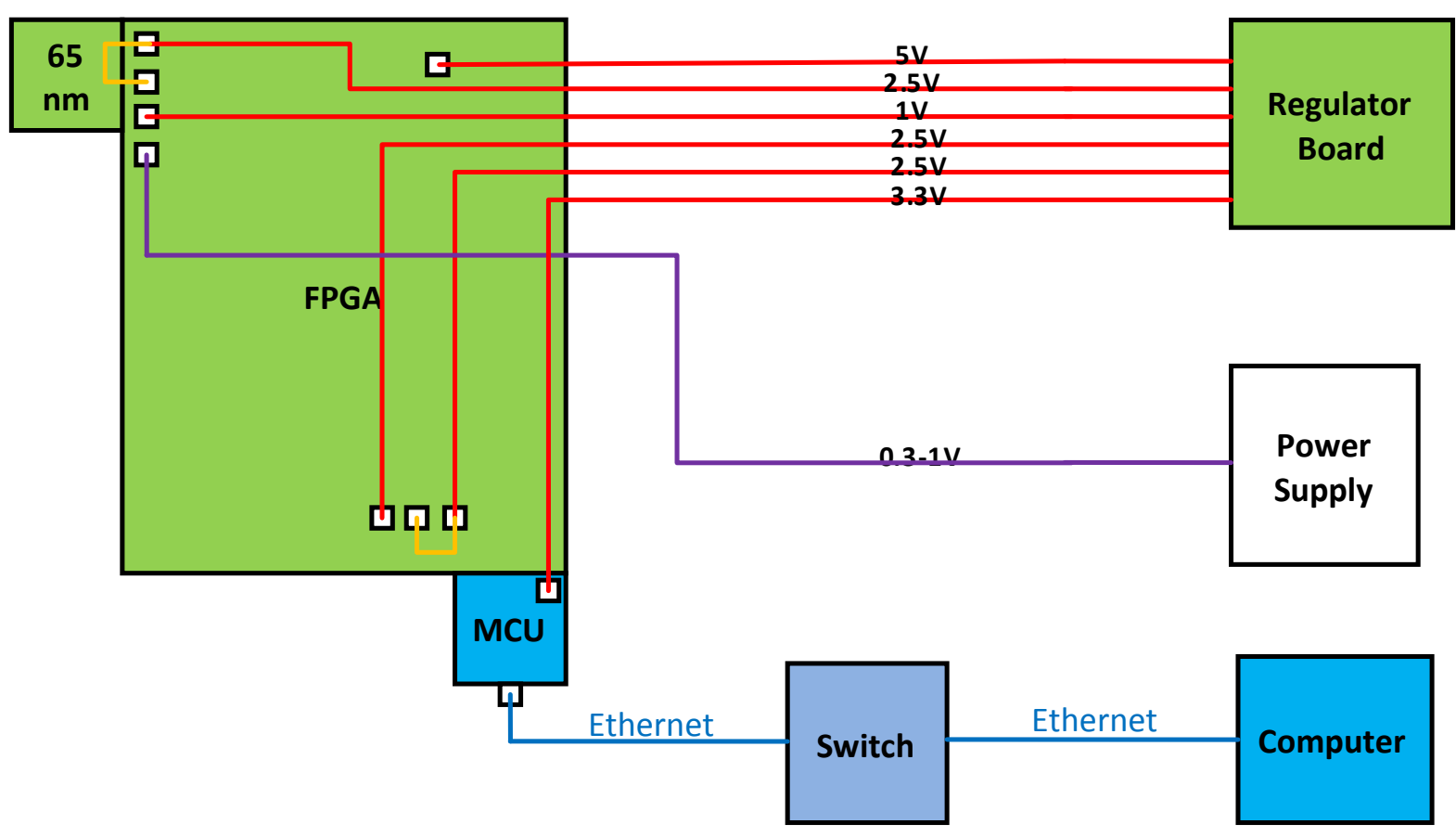

Figure 5.2 SRAM testing system setup 
At the beginning of the testing, data pattern, clock frequency, and SRAM array are selected by the configuration data file and loaded into the PC program. Following this, the configuration data is sent to the W7200MCU and then transferred to the PFGA. The FPGA generates the required Clock, write enable, read enable, address and data according to the configuration file, and is subsequently sent to the DUT. In addition, the FPGA samples outputs from the DUT at positive clock edge and sends the results back to the MCU. After the MCU receives all of the outputs from the targeted address, they are sent back to the computer through the ethernet cable. All of the inputs to the DUT, with the exception of supply power, are all 2.5 and all generated from the FPGA. The power supply to the SRAM is from the external power supply, ranging from $0.3 \mathrm{~V}$ to $1 \mathrm{~V}$.

\subsection{Functional Test}

In regards to a functional test with a variable supply voltage, the read and write functionality test with standard voltage $1 \mathrm{~V}$ was performed first, ensuring that the SRAM would perform adequately with the standard $1 \mathrm{~V}$. For the read and write test with a lower voltage than $1 \mathrm{~V}$, if same voltage was used for the read and write operation, it would be hard to determine whether it was a read error or write error. In this research, for the write test, the data was written to the SRAM at different voltages (0.3-1 V) and then read back at a high voltage $(1 \mathrm{~V})$ to ensure that the outputs were the same with the data stored in the memory cells. For the read test, data was written to the SRAM at a high voltage (1 V) to ensure the write data was written into the cells correctly, and subsequently read back at different voltages. In this case, when an error occurs, it is easy to identify whether it is a read or write error. 
Four different data patterns are written into the SRAM and then read back from the SRAM to investigate whether the contents are the same as what was written into the SRAM. The four data patterns are '00000000', '01010101', '10101010', and '11111111', respectively. These four data patterns are chosen as typical data patterns to test both ' 0 ' and ' 1 ' of each cell. The write and read procedure for one memory array is described in the following steps: First, set the supply power to I V and write the same data into the SRAM from lowest address (0) to highest address (511). Following this, read the contents out from address 0 to 511 . The testing results convey that with $\mathrm{V}_{D D}=1 \mathrm{~V}$, correct outputs can be obtained from all addresses for all four data patterns. Furthermore, it can be concluded that both read and write operations function as expected with the standard $1 \mathrm{~V}$ power supply.

After verifying the read and write functions with standard $V_{D D}$, read and write testing with lower supply voltages were performed. For the read testing, data was written into the SRAM with $V_{D D}=1 \mathrm{~V}$ first to ensure that all cells were written correctly. Following this, the supply voltage was changed to the target value and read from addresses from 0 to 511. For the write testing, data was written into the SRAM with targeted $V_{D D}$, and read from the SRAM with the standard power supply to ensure that all outputs read from the SRAM were synonymous with the data stored in the memory cells. All outputs were recorded and compared to the written data.

For the write functionality testing, the results showed that all addresses were written with correct data, with a power supply ranging from $1 \mathrm{~V}$ to $0.3 \mathrm{~V}$. This signifies that the SRAM is able to write the data correctly for sub-threshold and super-threshold voltages. 
For the read functionality test, data was written into all addresses with $V_{D D}=1 \mathrm{~V}$. The data read from the SRAM began to have errors when the supply voltage was lowered down to $700 \mathrm{mV}$. The testing results are shown in Table 5.1. Error rate for ' 0 ' means the possibility of getting ' 1 ' for content ' 0 ', while error rate for ' 1 ' means the possibility of getting ' 0 ' for content ' 1 '.

Table 5.1 Read error rates of the four cells from $0.3 \mathrm{~V}$ to $0.7 \mathrm{~V}$

\begin{tabular}{|c|c|c|c|c|c|c|}
\hline \multicolumn{7}{|c|}{ DICE } \\
\hline \multirow{2}{*}{0} & $V_{D D}$ & $700 \mathrm{mV}$ & $600 \mathrm{mV}$ & $500 \mathrm{mV}$ & $400 \mathrm{mV}$ & $300 \mathrm{mV}$ \\
\cline { 2 - 7 } & error number & 269 & 537 & 985 & 1709 & 2339 \\
\hline \multirow{2}{*}{1} & error rate (\%) & 6.57 & 13.11 & 24.05 & 41.72 & 57.104 \\
\cline { 2 - 7 } & error number & 45 & 156 & 343 & 708 & 1057 \\
\cline { 2 - 7 } & error rate (\%) & 1.10 & 3.81 & 8.37 & 17.29 & 25.81 \\
\hline
\end{tabular}

\begin{tabular}{|c|c|r|r|r|r|r|}
\hline \multicolumn{9}{|c|}{$10 \mathrm{~T}$} \\
\hline \multicolumn{2}{|c|}{$V_{D D}$} & $700 \mathrm{mV}$ & $600 \mathrm{mV}$ & $500 \mathrm{mV}$ & $400 \mathrm{mV}$ & $300 \mathrm{mV}$ \\
\hline \multirow{2}{*}{0} & error number & 0 & 128 & 128 & 336 & 1473 \\
\cline { 2 - 7 } & error rate (\%) & 0.00 & 3.13 & 3.13 & 8.20 & 35.96 \\
\hline \multirow{2}{*}{1} & error number & 0 & 3 & 64 & 192 & 551 \\
\cline { 2 - 7 } & error rate (\%) & 0.00 & 0.07 & 1.56 & 4.69 & 13.45 \\
\hline
\end{tabular}

\begin{tabular}{|c|c|r|r|r|r|r|}
\hline \multicolumn{7}{|c|}{ Quatro } \\
\hline \multicolumn{2}{|c|}{$V_{D D}$} & $700 \mathrm{mV}$ & $600 \mathrm{mV}$ & $500 \mathrm{mV}$ & $400 \mathrm{mV}$ & $300 \mathrm{mV}$ \\
\hline \multirow{2}{*}{0} & error number & 0 & 0 & 59 & 145 & 837 \\
\cline { 2 - 7 } & error rate (\%) & 0.00 & 0.00 & 1.44 & 3.54 & 20.43 \\
\hline \multirow{2}{*}{1} & error number & 0 & 0 & 0 & 0 & 317 \\
\cline { 2 - 7 } & error rate (\%) & 0.00 & 0.00 & 0.00 & 0.00 & 7.74 \\
\hline
\end{tabular}

\begin{tabular}{|c|c|r|r|r|r|r|}
\hline \multicolumn{9}{|c|}{$6 \mathrm{~T}$} \\
\hline \multicolumn{2}{|c|}{$V_{D D}$} & $700 \mathrm{mV}$ & $600 \mathrm{mV}$ & $500 \mathrm{mV}$ & $400 \mathrm{mV}$ & $300 \mathrm{mV}$ \\
\hline \multirow{2}{*}{0} & error number & 0 & 79 & 296 & 478 & 1224 \\
\cline { 2 - 7 } & error rate (\%) & 0.00 & 1.93 & 7.23 & 11.67 & 29.88 \\
\hline \multirow{2}{*}{1} & error number & 0 & 0 & 0 & 31 & 257 \\
\cline { 2 - 7 } & error rate (\%) & 0.00 & 0.00 & 0.00 & 0.76 & 6.27 \\
\hline
\end{tabular}


As is evident from the reading test results, DICE has the worst low-power performance, which is in conflict with previous simulation results. After analyzing the results and reviewing the design, a possible reason for the errors can be discerned.

Recall the schematic of the sense amplifier in chapter 3.1.5, which is designed to be dynamic rather than static. If $B L$ and $B L B$ did not set up enough of a potential difference when $S E N$ was asserted, then the results could be incorrect. In this design, SEQ and SEN signals are shorted, meaning that when the sense amplifier begins to work, the potentials on $B L$ and $B L B$ are still both equal to $V_{D D}$. At this point, small noise on $B L$ or $B L B$ may be captured by the sense amplifier, resulting in an incorrect output. If one was to recall the simulation results in Chapter 4.1.2, it is evident that DICE has the slowest read speed, rendering the sense amplifier for the DICE cell more susceptible to noise. Further evidence for the conclusion can be drawn from the fact that most of the errors were column based, meaning that the outputs from all cells connected to the same sense amplifier were incorrect when errors occurred. This further indicated that the phenomenon was due to the timing of the sense amplifier.

This problem may be resolved by adding some delay to $S E N$ signal after $S E Q$, which ensures that an adequate potential difference has been accumulated to overcome any possible noise in the bitlines. One simple way to apply delay to $S E N$ signal is to add a buffer chain prior to sending $S E N$ to the sense amplifier. 


\subsection{Radiation Test}

Although the SRAM was not fully functional with the subthreshold power supply, we can still find some columns that function for full $V_{D D}$ range from $0.4 \mathrm{~V}-1 \mathrm{~V}$, and for both input ' 0 ' and input ' 1 '. After discerning the "correct" columns, a radiation test is performed to assess the radiation performance of cells with different supply voltages. The test system is programmed to write '00000000' to addresses from ' $\mathrm{xxx} 000000$ ' to 'xxx111111', and write '1111111' to addresses from 'xxx100000' to 'xxx1111111.' Following this, the system waits for 4 seconds and reads the content from the test chip following the sequence of DICE, 10T, Quatro, and 6T, from address 0 to 511. After reading out the data from all of the addresses, the data is refreshed again to recover the "upset" bits. Then, the whole cycle is repeated until preconfigured testing cycles are reached. When the computer receives the outputs from SRAM, "bad" columns, which have been detected through functional tests, are masked; only "good" columns are compared with input data to determine if SEUs occurred in that this 4-second time period. Error bit maps, which show if an upset occurs on each bit (physical location), are constructed. By analyzing the error bit maps, single-bit upset (SBU) and multi-bit upset (MBU) can be distinguished.

In the common SRAM design, memory cells are placed as tightly as possible to obtain high density [10]. As the feature size becomes smaller in advanced technology, adjacent cells become closer. For this reason, single-event induced charge is able to spread to an area that is larger than that of one cell and multi-node upsets (MNUs) and multi-bit upsets (MBUs) may occur [11]-[14]. Memory cells are even more sensitive to particle strikes if supply voltage 
reduction is taken into consideration, and MNUs and MBUs probabilities could be predicted to be higher.

In this thesis, single bit upset and multi bit upset are defined as in Figure 5.3. The figure shows nine cells with their physical location. The black dot in the middle cell signifies that an upset occurs at that bit. In the left figure, blank signifies that the other 8 adjacent cells are all correct, and this upset is considered a single cell upset. In the right figure, if an upset occurs at any one of the 8 adjacent cells, indicated by X, it is considered a multi bit upset.

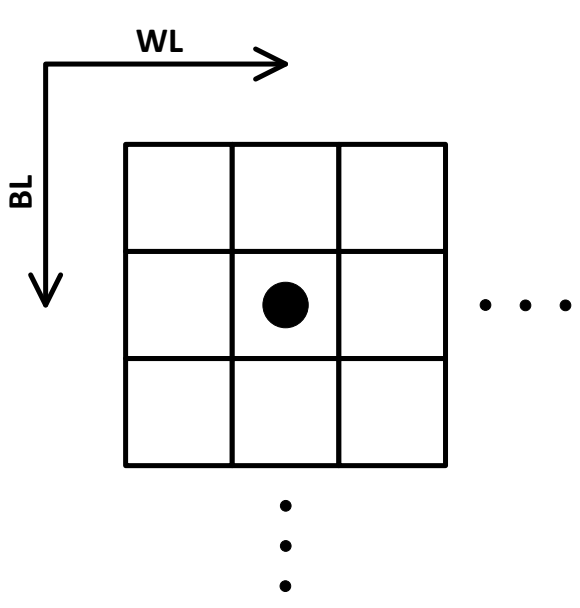

(a) Single-bit upset

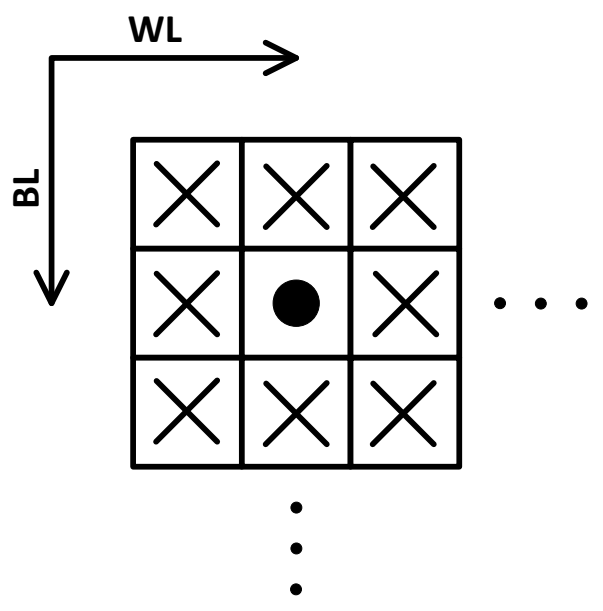

(b) Multi-bit upset

Figure 5.3 SBU and MBU definition

\subsubsection{Alpha Radiation Test}

First, an alpha radiation source is applied right above the chip. The flux of the alpha particle source is $4.61 \times 10^{7} \mathrm{~cm}^{-2} \mathrm{~h}^{-1}$. For each supply voltage, the chip was radiated for 15 minutes, and the upset number was counted for each SRAM array. Error rate was calculated as the number of upsets per bit in 15 minutes. Testing results are listed in Table 5.2. 
Table 5.2 Alpha particle radiation test result

\begin{tabular}{|l|r|r|r|r|r|}
\hline DICE & '0'->'1' error & '1'->'0' error & total error & capacitance & Error rate (\%) \\
\hline $1000 \mathrm{mV}$ & 0 & 0 & 0 & 4096 & 0.00 \\
\hline $900 \mathrm{mV}$ & 0 & 0 & 0 & 4096 & 0.00 \\
\hline $800 \mathrm{mV}$ & 0 & 0 & 0 & 3904 & 0.00 \\
\hline $700 \mathrm{mV}$ & 0 & 1 & 1 & 3648 & 0.03 \\
\hline $600 \mathrm{mV}$ & 0 & 0 & 0 & 3200 & 0.00 \\
\hline $500 \mathrm{mV}$ & 0 & 0 & 0 & 2304 & 0.00 \\
\hline $400 \mathrm{mV}$ & 5 & 4 & 9 & 1472 & 0.61 \\
\hline
\end{tabular}

\begin{tabular}{|l|r|r|r|r|r|}
\hline $10 \mathrm{~T}$ & '0'->'1' error & '1'->'0' error & total error & capacitance & Error rate (\%) \\
\hline $1000 \mathrm{mV}$ & 239 & 247 & 486 & 4096 & 11.87 \\
\hline $900 \mathrm{mV}$ & 278 & 269 & 547 & 4096 & 13.35 \\
\hline $800 \mathrm{mV}$ & 327 & 315 & 642 & 4032 & 15.92 \\
\hline $700 \mathrm{mV}$ & 343 & 344 & 687 & 4032 & 17.04 \\
\hline $600 \mathrm{mV}$ & 367 & 381 & 748 & 3968 & 18.85 \\
\hline $500 \mathrm{mV}$ & 421 & 408 & 829 & 3840 & 21.59 \\
\hline $400 \mathrm{mV}$ & 461 & 480 & 941 & 3648 & 25.79 \\
\hline
\end{tabular}

\begin{tabular}{|l|r|r|r|r|r|}
\hline \multicolumn{1}{|c|}{ Quatro } & '0'->'1' error & '1'->'0' error & total error & capacitance & Error rate (\%) \\
\hline $1000 \mathrm{mV}$ & 0 & 1 & 1 & 4096 & 0.02 \\
\hline $900 \mathrm{mV}$ & 3 & 1 & 4 & 4096 & 0.10 \\
\hline $800 \mathrm{mV}$ & 4 & 5 & 9 & 4096 & 0.22 \\
\hline $700 \mathrm{mV}$ & 24 & 30 & 54 & 4096 & 1.32 \\
\hline $600 \mathrm{mV}$ & 52 & 40 & 92 & 3904 & 2.36 \\
\hline $500 \mathrm{mV}$ & 99 & 56 & 155 & 3520 & 4.40 \\
\hline $400 \mathrm{mV}$ & 102 & 79 & 181 & 3136 & 5.77 \\
\hline
\end{tabular}

\begin{tabular}{|c|r|r|r|r|r|}
\hline $6 \mathrm{~T}$ & '0'->'1' error & '1'->'0' error & total error & capacitance & Error rate (\%) \\
\hline $1000 \mathrm{mV}$ & 327 & 315 & 642 & 4096 & 15.67 \\
\hline $900 \mathrm{mV}$ & 333 & 337 & 670 & 4096 & 16.36 \\
\hline $800 \mathrm{mV}$ & 392 & 364 & 756 & 4096 & 18.46 \\
\hline $700 \mathrm{mV}$ & 396 & 422 & 818 & 4096 & 19.97 \\
\hline $600 \mathrm{mV}$ & 479 & 467 & 946 & 4096 & 23.10 \\
\hline $500 \mathrm{mV}$ & 498 & 526 & 1024 & 4032 & 25.40 \\
\hline $400 \mathrm{mV}$ & 560 & 591 & 1151 & 3840 & 29.97 \\
\hline
\end{tabular}

From the alpha testing result, we can come up some conclusions: 
1. The possibility of an '0'->'1' and ' 1 ' $->$ ' 0 ' upset is almost the same, which is in accordance with the symmetric structure of memory cells.

2. Error rate for $10 \mathrm{~T}$ cells is slightly smaller than the 6T cell. Both $6 \mathrm{~T}$ and $10 \mathrm{~T}$ cells have much larger error rates than DICE and Quatro cells; this result matches the critical charge simulation results in 4.1.2.

3. Quatro cells are more SEU susceptible than DICE cells with alpha source radiation. This is in agreement with the simulation results, which convey that DICE cell does not flip with a single node strike, while two out of eight configurations of the Quatro cell will lead to SEUs.

\subsubsection{Heavy Ion Radiation Test}

After the chip was tested using an alpha radiation source, heavy ion radiation experiments were performed at China Institute of Atomic Energy (CIAE). Heavy ions are very significant tools to study the SEE, as they are ubiquitous in the space. LET (unit $\mathrm{MeV} \cdot \mathrm{cm}^{2} / \mathrm{mg}$ ) describes the energy that a charged ionizing particle transfers to the material per unit distance. The LETs of the heavy ions are related to both the ion types and the ion energies. The same testing system and procedure as alpha testing is used for heavy ion testing. The experiment setup for heavy ion testing in CIAE is displayed in Figure 5.4 and Figure 5.5. Five heavy ions with different LET ranging from 0.44 to $22 \mathrm{MeV} \cdot \mathrm{cm}^{2} / \mathrm{mg}$ were selected. The parameters of the heavy ions applied are listed in Table 5.3.

Only the DUT, FPGA board and MCU controller were placed in the vacuum chamber.

The regulator board, power supplies, switch and computers are located in the radiation room, which is outside the chamber. Connections between the radiation room and vacuum chamber 
are through female DB25 connectors on the chamber board. Another computer, named computer_2, was used in the control room to remote control computer_1 in the test room.

Table 5.3 Heavy Ions Parameters

\begin{tabular}{|c|c|c|c|c|}
\hline Particle & $\begin{array}{c}\text { Energy } \\
(\mathrm{MeV})\end{array}$ & $\begin{array}{c}\text { LET } \\
\left(\mathrm{MeV} \cdot \mathrm{cm}^{2} / \mathrm{mg}\right)\end{array}$ & $\begin{array}{c}\text { Range } \\
(\mu \mathrm{m})\end{array}$ & $\begin{array}{c}\text { Flux } \\
\left(\# / \mathrm{cm}^{2} / \mathrm{s}\right)\end{array}$ \\
\hline $\mathrm{Li}$ & 45 & 0.44 & 259.6 & $1 \times 10^{6}$ \\
\hline $\mathrm{C}$ & 80 & 1.73 & 127 & $4.3 \times 10^{5}$ \\
\hline $\mathrm{F}$ & 110 & 4.2 & 82.7 & $3.4 \times 10^{4}$ \\
\hline $\mathrm{Si}$ & 140 & 9.1 & 53 & $2.9 \times 10^{5}$ \\
\hline $\mathrm{Ti}$ & 165 & 22 & 33.9 & $5.2 \times 10^{5}$ \\
\hline
\end{tabular}

Vacuum Chamber

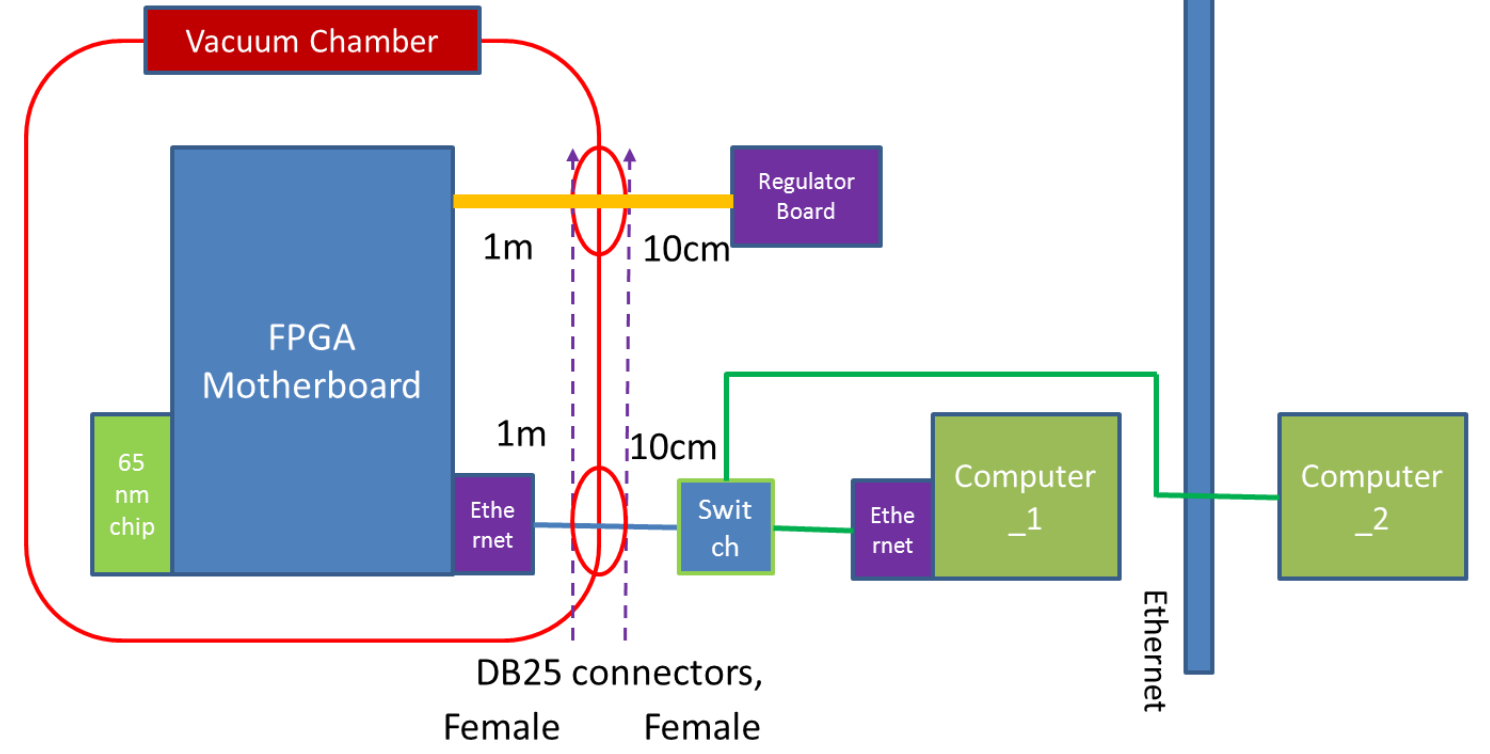

Figure 5.4 Heavy ion test environment setup 


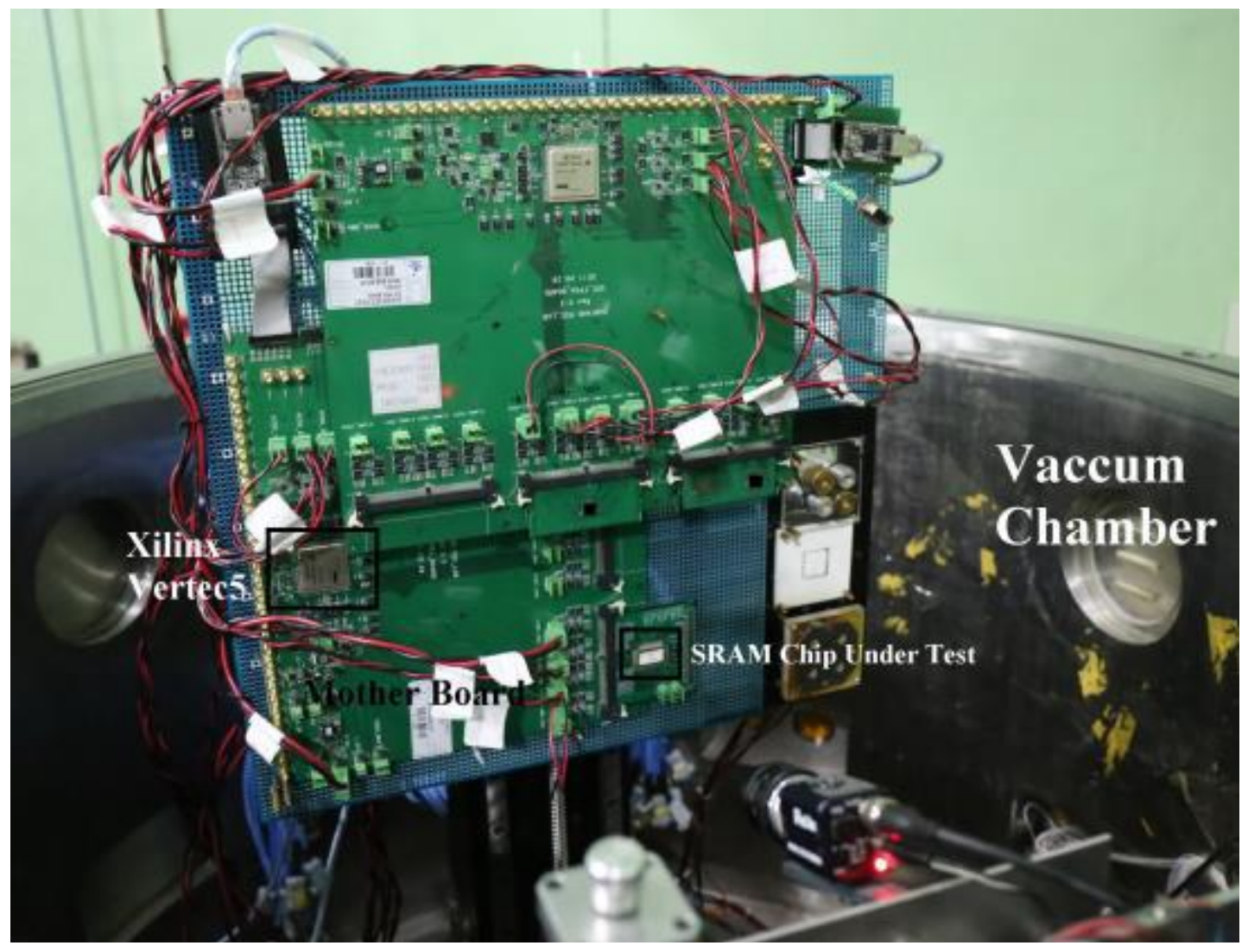

Figure 5.5 Heavy ion test system

During the testing, the selected particles were ionized, accelerated, and struck on the test chip. LET and fluence (which is defined as the total number of particles in a unit area, unit $\mathrm{cm}^{-2}$ ) of the charged ionizing particle, bits capacitance of the SRAM chip, the number of SEUs, and locations of the errors were recorded by the computer.

\subsubsection{Cross Section Analysis}

In nuclear science and physics, cross section is used to express the possibility of interaction between particles. In SEU study, cross section per bit can be used to describe the SEU sensitivity, and is expressed by SEU error numbers vs. fluence vs. capacitance, as defined in equation 5.1 . 


$$
\sigma=N_{\text {Event }} /(F * V)
$$

Here, $N_{\text {Event }}$ is total amount of upsets, $F$ is particle fluence, and $V$ is the volume of irradiated devices (size of an SRAM array). The particle fluence $F$ and volume $V$ can be treated as known and fixed parameters. The random characteristic of cross section comes from $N_{\text {event }}$. Under a similar radiation condition, the amount of upsets of digital cells is a discrete random variable, which follows Poisson distribution with an expectation $\lambda$ and variance $\sigma^{2}$. According to Central Limit Theorem, the number of tests times is large enough, Poisson distribution can be considered as Normal distribution. For Normal distribution, $2 \sigma$ error bar means that the random variable has a probability of $95.4 \%$ to remain in the $[\mu-2 \sigma, \mu+2 \sigma]$ range.

From equation 5.1, cross section per bit with a $2 \sigma$ error bar can be calculated for each test case and the results are outlined in Figure 5.4. In the figure, data points at each supply voltage are purposely offset in the horizontal direction to distinguish the corresponding error bars. Zero data points, which signify that no SEU occurs, are indicated with arrows pointing to the $\mathrm{x}$ axis. 


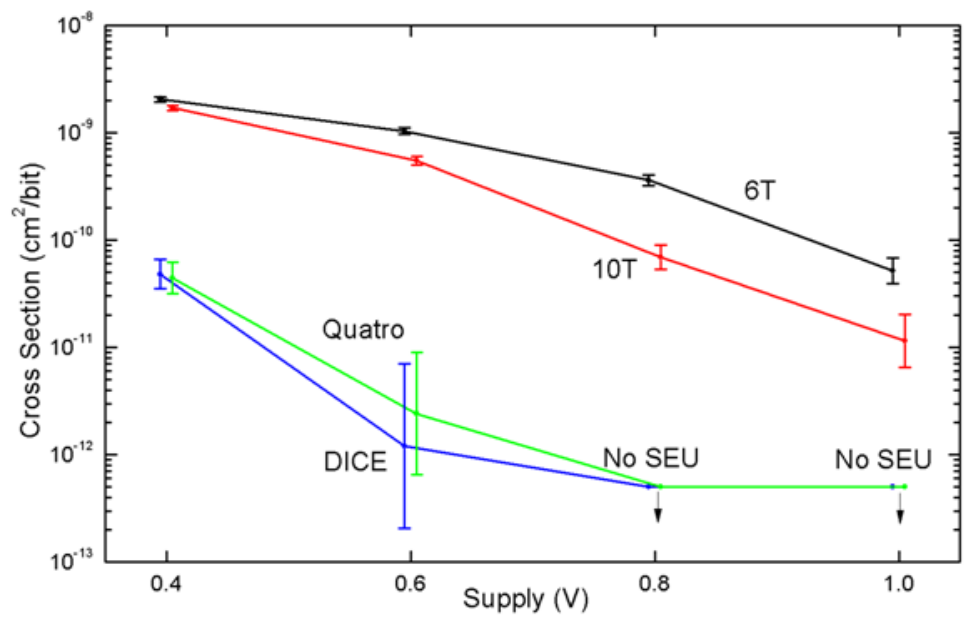

(a) $\mathrm{LET}=0.44 \mathrm{MeV} \cdot \mathrm{cm} 2 / \mathrm{mg}$

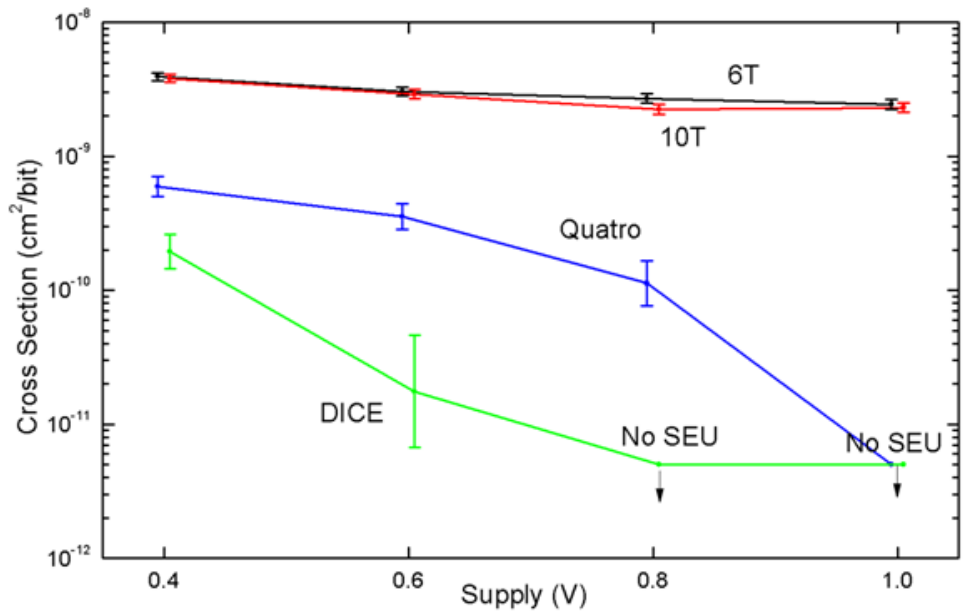

(b) $\mathrm{LET}=1.73 \mathrm{MeV} \cdot \mathrm{cm} 2 / \mathrm{mg}$ 


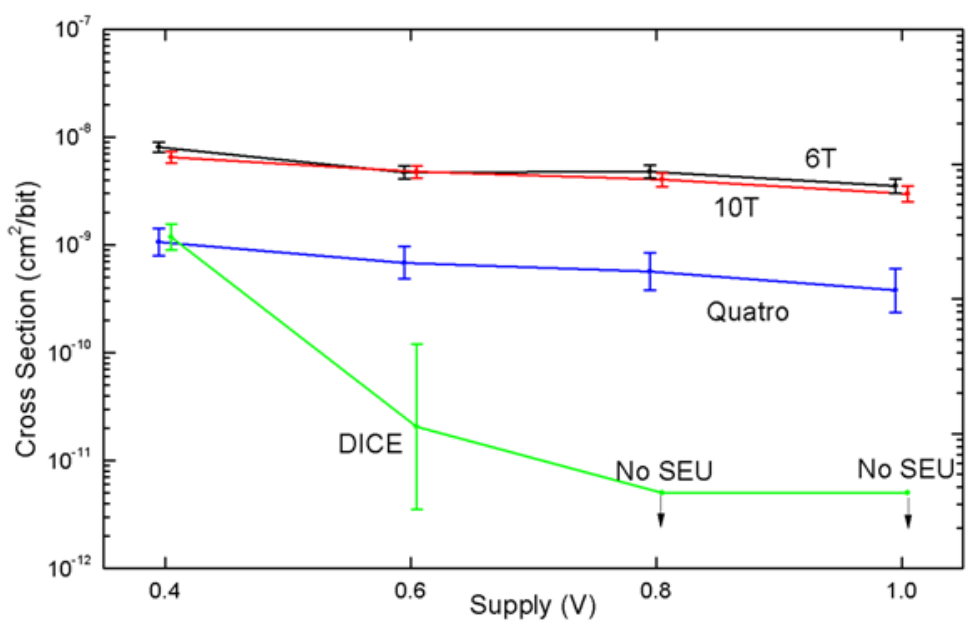

(c) $\mathrm{LET}=4.2 \mathrm{MeV} \cdot \mathrm{cm} 2 / \mathrm{mg}$

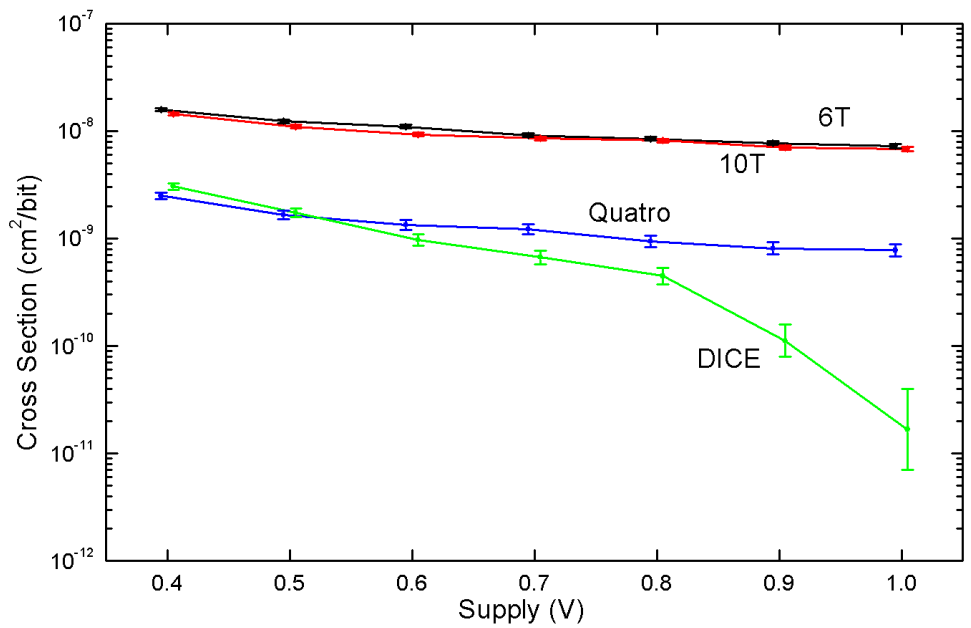

(d) $\mathrm{LET}=9.1 \mathrm{MeV} \cdot \mathrm{cm} 2 / \mathrm{mg}$ 


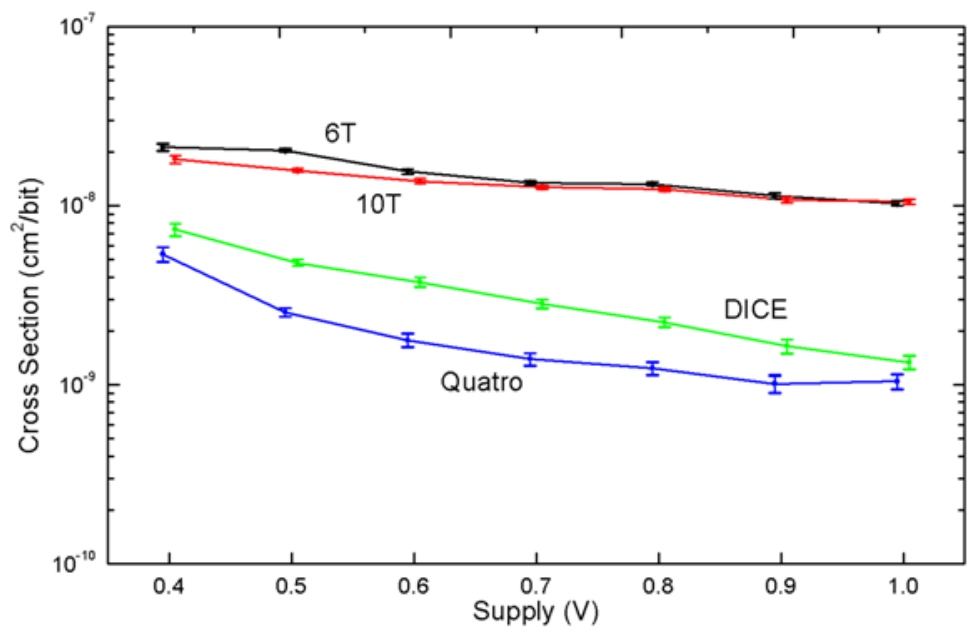

(e) $\mathrm{LET}=22 \mathrm{MeV} \cdot \mathrm{cm}^{2} / \mathrm{mg}$

Figure 5.6 Cross section of the four SRAM cell arrays.

Results are analyzed as following:

1. For all types of SRAM cells, as an expected result, the cross section increases as the supply voltage decreases. The results are also in accordance with an alpha radiation test and critical charge simulation results.

2. Cross sections of two radiation-tolerant cells (DICE and Quatro) are obviously lower than those of two un-hardened cells (6T and 10T) at each testing condition, meaning that these two RHBD designs can effectively reduce soft error rates, even at low supply voltages.

3. From Figure 5.6 (a) it is evident that, when irradiated by lower energy ions ( $\mathrm{Li}$, in this study, LET $=0.44 \mathrm{MeV} \cdot \mathrm{cm}^{2} / \mathrm{mg}$ ), $6 \mathrm{~T}$ cells present higher cross sections than $10 \mathrm{~T}$ cells. This difference was especially obvious when supply voltages are high. One 
possible explanation is due to the extra transistors in $10 \mathrm{~T}$ cells. Recall the schematic of the 6T cell and 10T cell in Figure 3.3 and Figure 3.4. As compared to the 6T cell, the $10 \mathrm{~T}$ cell has one additional transistor at each side, named $N 5$ and $N 6$, which are connected to storage nodes $Q$ and $Q B$, respectively. According to equation 4.1, increased capacitance on the node will consequently increase the critical charge. This additional capacitance enhances radiation tolerance of $10 \mathrm{~T}$ cells compared to $6 \mathrm{~T}$ cells. This benefit is especially obvious when supply voltage is $1 \mathrm{~V}$, say a 4.5 times smaller cross section. However, in cases of larger LETs and smaller supply voltages, this benefit becomes almost negligible, which also explains the fact that the differences between cross sections of $6 \mathrm{~T}$ and $10 \mathrm{~T}$ not significant enough for other ions except Li. For all other particles except $\mathrm{Li}$, the $2 \sigma$ error bars associated with these data points of $6 \mathrm{~T}$ and $10 \mathrm{~T}$ cell are largely overlapped. Hence, it is difficult to determine which one is more reliable than the other according to this data.

4. In Figure $5.6(a, b, c)$, it can be observed that with relatively low LET (Li, C, F), cross sections of the DICE cell are smaller than the Quatro cell, especially when the supply voltage is larger than $0.6 \mathrm{~V}$. Based on the results, it can be concluded that the DICE cell is more reliable than the Quatro cell with lower energy particles. This can be explained by single-node upsets, as described in Chapter 4.1.2. Unlike the DICE cell, the Quatro cell is not fully single-node upset tolerant.

In Figure 5.4 (d), when supply voltage goes under $0.6 \mathrm{~V}$, the cross section of DICE cells show no significant advance compared to Quatro cells. When it comes to high LET radiation conditions, for example, $\mathrm{LET}=22 \mathrm{MeV} \cdot \mathrm{cm}^{2} / \mathrm{mg}$ (e), DICE cells begin to lose in 
the reliability competition with Quatro cells for all power supply ranges, as shown in Figure 5.4(e). This phenomenon can be explained by SEEs charge spreading, which could induce multi-node upsets (MNUs) inside a single cell and multi-bit upsets (MBUs) in the array. DICE cells are not multi-node upset tolerant, since DICE presents more sensitive node pairs than Quatro for double-node upsets [27]. Both MNUs and MBUs would obviously degrade the single-event robustness of SRAM arrays and lead to larger cross sections.

MNUs inside a memory cell cannot be identified, however, MBUs can be quite easily observed, considering the fact that the location of each cell upset in each read cycle can be recognized and recorded. Based on the experimental results, cross sections induced by SBUs and MBUs were further separated to observe contribution of SBUs and MBUs to the total cross sections shown in Table 5.4. For LET $=22 \mathrm{MeV} \cdot \mathrm{cm}^{2} / \mathrm{mg}$, MBUs induced cross sections of DICE cells are larger than those of Quatro cells with most supply voltages, especially in a low supply voltage range. This result indicates that Quatro cells are more robust to charge spreading than DICE cells, which also supports the conclusion given above. From Table 5.4, it can be discerned that the DICE cells also have larger SBUs contributed cross sections for all supply voltages when LET=22 $\mathrm{MeV} \cdot \mathrm{cm}^{2} / \mathrm{mg}$. According to the SBUs cross sections data, it can be inferred that the MNUs inside the DICE cell play an indispensable role to its single-event robustness degradation, which is consistent with the work presented in [27]. 
Table 5.4 SBUs and MBUs Cross Sections of the Four SRAM Cell Arrays

\begin{tabular}{|c|c|c|c|c|c|c|c|c|c|}
\hline \multicolumn{10}{|c|}{ SBUs / MBUs Cross Sections $\left(10^{-10} \mathrm{~cm}^{2} / \mathrm{bit}\right)$} \\
\hline \multirow{2}{*}{$\begin{array}{c}\text { LET } \\
\left(\mathrm{MeV} \cdot \mathrm{cm}^{2} / \mathrm{mg}\right)\end{array}$} & \multirow{2}{*}{$\begin{array}{c}\text { Supply } \\
\text { Voltage } \\
\text { (V) }\end{array}$} & \multicolumn{2}{|c|}{$6 \mathrm{~T}$} & \multicolumn{2}{|c|}{$10 \mathrm{~T}$} & \multicolumn{2}{|c|}{ Quatro } & \multicolumn{2}{|c|}{ DICE } \\
\hline & & SBUs & MBUs & SBUs & MBUs & SBUs & MBUs & SBUs & MBUs \\
\hline \multirow{4}{*}{0.44} & 1 & 0.517 & 0 & 0.115 & 0 & 0 & 0 & 0 & 0 \\
\hline & 0.8 & 3.61 & 0 & 0.689 & 0 & 0 & 0 & 0 & 0 \\
\hline & 0.6 & 9.85 & 0.532 & 5.35 & 0.121 & 0.0121 & 0 & 0.024 & 0 \\
\hline & 0.4 & 19.3 & 1.17 & 16.0 & 0.122 & 0.481 & 0 & 0.44 & 0 \\
\hline \multirow{4}{*}{1.73} & 1 & 23.8 & 0.675 & 22.6 & 0.464 & 0 & 0 & 0 & 0 \\
\hline & 0.8 & 26.4 & 0.519 & 22.0 & 0.440 & 1.13 & 0 & 0 & 0 \\
\hline & 0.6 & 29.6 & 0.789 & 28.6 & 0.438 & 3.55 & 0 & 0.175 & 0 \\
\hline & 0.4 & 37.4 & 1.90 & 36.2 & 1.90 & 5.93 & 0 & 1.95 & 0 \\
\hline \multirow{4}{*}{4.2} & 1 & 35.4 & 0 & 29.5 & 0.421 & 3.79 & 0 & 0 & 0 \\
\hline & 0.8 & 47.7 & 0 & 40.2 & 0 & 6.56 & 0 & 0 & 0 \\
\hline & 0.6 & 47.2 & 0 & 47.6 & 3.20 & 6.83 & 0 & 0.21 & 0 \\
\hline & 0.4 & 78.2 & 2.37 & 64.9 & 4.74 & 9.96 & 0.711 & 11.8 & 0 \\
\hline \multirow{7}{*}{9.1} & 1 & 66.1 & 6.41 & 62.6 & 5.67 & 7.71 & 0.0671 & 0.168 & 0 \\
\hline & 0.9 & 70.1 & 7.60 & 65.0 & 5.39 & 8.10 & 0 & 1.12 & 0 \\
\hline & 0.8 & 76.6 & 8.21 & 74.4 & 7.08 & 9.03 & 0.342 & 4.48 & 0 \\
\hline & 0.7 & 83.2 & 8.45 & 76.1 & 9.37 & 11.5 & 0.677 & 6.56 & 0.14 \\
\hline & 0.6 & 97.5 & 13.1 & 82.7 & 10.4 & 12.8 & 0.576 & 9.61 & 0.11 \\
\hline & 0.5 & 10.5 & 18.1 & 97.0 & 13.5 & 16.1 & 0.588 & 16.4 & 0.88 \\
\hline & 0.4 & 13.0 & 28.0 & 121 & 23.0 & 23.7 & 1.23 & 27.9 & 2.74 \\
\hline \multirow{7}{*}{22} & 1 & 82.8 & 20.4 & 84.4 & 21.1 & 10.2 & 0.208 & 13.0 & 0.391 \\
\hline & 0.9 & 88.4 & 25.5 & 85.4 & 22.4 & 9.83 & 0.323 & 15.6 & 0.84 \\
\hline & 0.8 & 100 & 11.7 & 94.7 & 28.6 & 11.6 & 0.828 & 21.1 & 1.29 \\
\hline & 0.7 & 99.7 & 34.4 & 96.3 & 30.3 & 13.4 & 0.560 & 26.2 & 2.11 \\
\hline & 0.6 & 103 & 52.2 & 98.5 & 38.7 & 16.9 & 0.878 & 34.3 & 3.14 \\
\hline & 0.5 & 111 & 93.0 & 102 & 55.7 & 23.5 & 1.89 & 43.2 & 4.76 \\
\hline & 0.4 & 118 & 94.3 & 105 & 76.4 & 41.9 & 11.8 & 65.1 & 8.56 \\
\hline
\end{tabular}

\subsubsection{Bit-Cell Upsets Distributions Analysis}

As described in Chapter 5.1, the error bit map with the location of each cell upset was recorded in each read cycle. In this case, the total number of upsets occurring in each cell can be obtained by accumulating each error bit map together to analyze the distribution of upsets 
in each SRAM array during each test. The probability distribution of upsets in a SRAM array is expected to follow Poisson distribution [30], [31].

According to the expression of Poisson distribution as equation 5.2,

$$
P\left(\mathrm{~N}_{\text {upset }}=k\right)=\frac{\lambda^{k}}{k !} \times e^{-\lambda}, k=0,1,2 \ldots
$$

the mathematical expectation of Poisson distribution can be calculated as

$$
\lambda=-\ln \left(\frac{N_{\text {no_upset }}}{N_{\text {total }}}\right)
$$

Here, $N_{\text {no_upset }}$ is the number of cells not upset during radiation test, and $N_{\text {total }}$ is the number of total cells. Using $N_{\text {no_upset }}$ and $N_{\text {total }}$, mathematical expectation $\lambda$ and the probability distribution of all numbers of upsets can be calculated. To verify the assumption that the number of upsets is in accordance with Poisson distribution, some test data was randomly chosen and analyzed. The upset probability distributions are plotted in Fig.5.8.
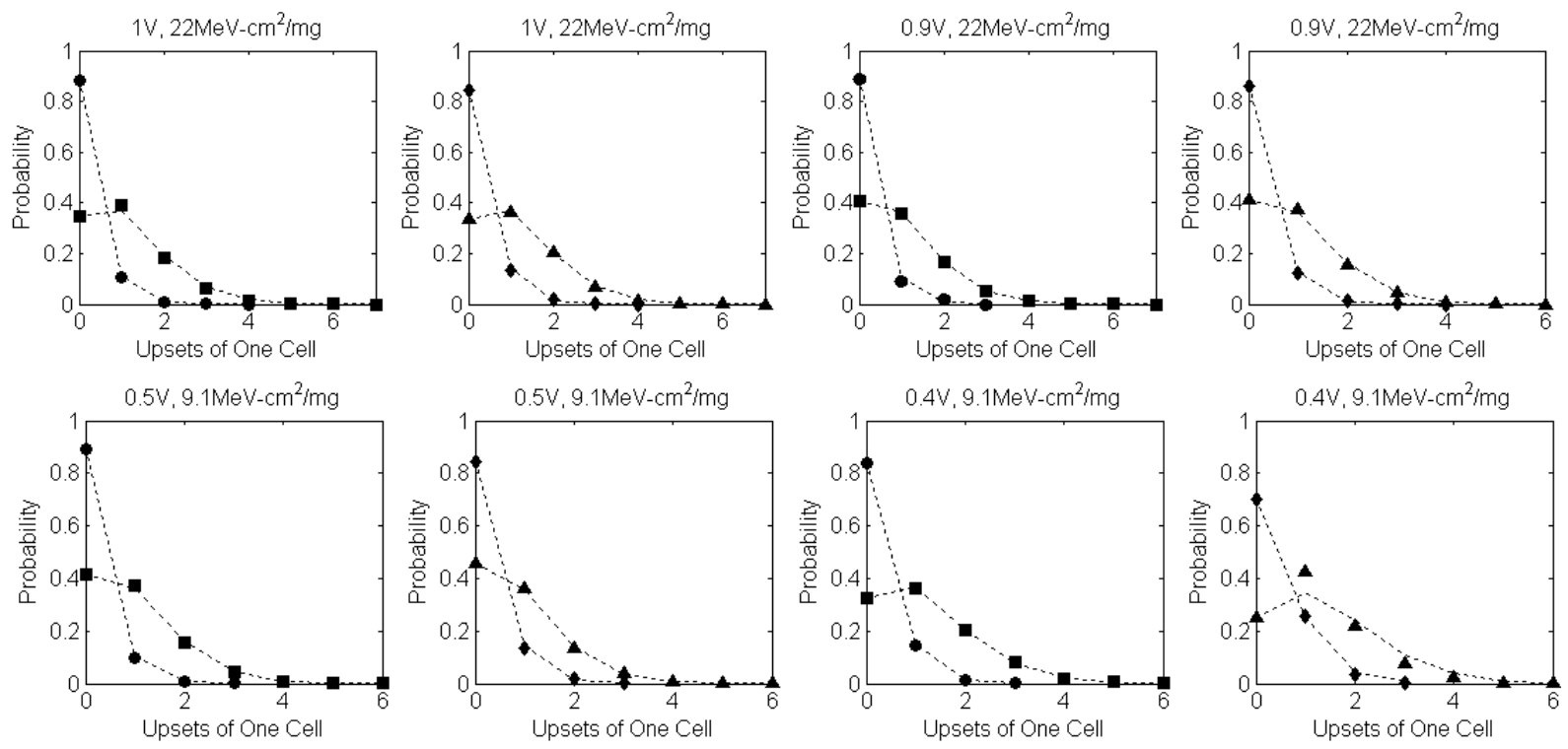

Figure 5.8 Bit-cell upsets distributions of SRAMs 
These figures present the probability distribution of four cells in four conditions, $1 \mathrm{~V}$ with $\mathrm{LET}=22 \mathrm{MeV} \cdot \mathrm{cm}^{2} / \mathrm{mg}, 0.9 \mathrm{~V}$ with $\mathrm{LET}=22 \mathrm{MeV} \cdot \mathrm{cm}^{2} / \mathrm{mg}, 0.5 \mathrm{~V}$ with $\mathrm{LET}=9.1$ $\mathrm{MeV} \cdot \mathrm{cm}^{2} / \mathrm{mg}$, and $0.5 \mathrm{~V}$ with $\mathrm{LET}=9.1 \mathrm{MeV} \cdot \mathrm{cm}^{2} / \mathrm{mg}$, In all sub-figures, the symbols

, $\boldsymbol{\Delta}$, and $\diamond$ represent data points of 6T, Quatro, 10T, and DICE, respectively. The solid dots represent the probability distribution discerned from tests results, while the dotted lines show the corresponding Poisson distributions theoretically predicted from equations 5.1 and 5.2 .

As depicted in Figure 5.8, all of the experimental data is in accordance with the respective theoretical probability distributions. In fact, the expectation $\lambda$ obtained can also be used to describe the SEUs sensitivity memory cell operated and irradiated in a certain condition. A larger $\lambda$ indicates a lower SEU robustness. The test and calculated results presented in Figure 5.8 further substantiate that Poisson distribution can be employed as a tool to describe the statistic behavior of bit-cell upsets in SRAM arrays, which could also build a foundation for applying Poisson distribution to generate SEU-like fault injections for simulations. 


\section{CHAPTER 6 \\ CONCLUSION AND FUTURE WORK}

\subsection{Conclusions}

To study the supply voltage dependence of SEU on SRAM, this work presents the alpha and heavy ion test results of four types of SRAMs: 6T, 10T, Quatro, and DICE, fabricated on a $65 \mathrm{~nm} 1 \mathrm{~V}$ process. These SRAMs have been operated with different supply voltages ranging from standard $1 \mathrm{~V}$ to sub-threshold $0.4 \mathrm{~V}$, and also irradiated by alpha particles and heavy ions with various LETs $\left(0.44,1.73,4.2,9.1\right.$, and $\left.22 \mathrm{MeV} \cdot \mathrm{cm}^{2} / \mathrm{mg}\right)$. Both alpha and heavy ion radiation results demonstrate accordance with the simulation results. Single-bit upset and multi-bits upset on four types of memory cells, as well as the bits-cell upset distribution were also investigated and analyzed.

One common trend for all SRAMs is the fact that the decrease of supply voltage increases their sensitivities to SEEs, which is mainly a result of the reduction of critical charge. This trend should be given more attention, considering the fact that low voltage operation is currently a popular method for large scale SRAMs design to reduce power, and SRAMs' increased SEEs susceptibilities can be a serious threat to reliable applications in radiation environments.

According to our test results, the radiation-hardened DICE and Quatro cells remain a more reliable option than the unhardened $6 \mathrm{~T}$ and $10 \mathrm{~T}$ cells in the near sub-threshold and sub-threshold regions. The winner of the reliability competition between DICE and Quatro depends on both supply voltage and LET. With lower LETs, DICE presents more optimal 
reliabilities, considering the fact that it is completely hardened against single-node upsets, whereas Quatro cell is not. However, in the case where LET increases to $9.1 \mathrm{MeV} \cdot \mathrm{cm}^{2} / \mathrm{mg}$ and supply voltage drops to lower than $0.6 \mathrm{~V}$, or LET=22 $\mathrm{MeV} \cdot \mathrm{cm}^{2} / \mathrm{mg}$ for all supply voltages, Quatro performs better than DICE. Analysis on SBUs and MBUs revealed that the possible explanation for this phenomenon is that the DICE cell operated with relatively lower supply voltages and/or irradiated with relatively higher LETs demonstrates more consequential sensitivities to SEEs charge spreading than the Quatro cell. Charge spreading induced MNUs and MBUs finally contribute to the larger cross sections of DICE.

Poisson distribution is applied to analyze the statistic behavior of bit-cell upsets in all SRAMs. There is evidence that the actual upset distributions in the four SRAM arrays fit their theoretically predicted Poisson distributions very well. This conclusion can also serve as the foundation of applying Poisson distribution to generate SEU-like fault injections for simulations.

\subsection{Future work}

In this work, the SRAM was not fully functional in a subthreshold region. Thus, when the supply voltage was lower than $0.7 \mathrm{~V}$, functional errors began to occur. This thesis analyzed the results and proposed an improvement strategy in order to resolve this problem. Future research should be conducted to improve the design and make it fully functional for all superthreshold and subthreshold voltages.

This paper explored the supply voltage dependence of SEU in four different cells. The new radiation hardened cell by design and the new radiation tolerant cell by layout technique will be a promising work, utilizing the SRAM structure and test system. 
The capacity of the SRAM is $4 \mathrm{~K} \times 4$ bits in this project, and significantly smaller than SRAM used in modern digital systems. This is especially applicable in cases of signal processing and data collection systems, both of which require a large capacity SRAM. Therefore, designing subthreshold SRAM with larger capacity is also an interesting research objective that can be explored in the future. 


\section{REFERENCES}

[1] Andrei Pavlov and Manoj Sachdev, CMOS SRAM Circuit Design and Parametric Test in Nano-Scaled Technologies, Springer, 2008, pp.1-2.

[2] Neil H.E.Weste and David Money Harris, Integrated Circuit Design (Fourth Edition), Publishing House of Electronics Industry, 2011, pp.475-478.

[3] Benton H. Calhoun and Anantha P. Chandrakasan, "Static Noise Margin Variation for Sub-threshold SRAM in 65-nm CMOS”, IEEE J. Solid-State Circuits, vol. 41, no.7, pp. 1673- 1679, July. 2006.

[4] Benton H. Calhoun and Anantha Chandrakasan, "A 256kb Sub-threshold SRAM in 65nm CMOS”, in IEEE ISSCC Dig. Tech. Papers, 2006, pp.2592-2601.

[5] Benton Highsmith Calhoun and Anantha P. Chandrakasan, “A 256-kb 65-nm Sub-threshold SRAM Design for Ultra-Low-Voltage Operation", IEEE J. Solid-State Circuits, vol.42, no.3, pp. 680-688, Mar. 2007.

[6] Sina Hassanzadeh, Milad Zamani, Khosrow Hajsadeghi, and Roghayeh Saeidi, “A Novel Low Power 8T-cell Sub-threshold SRAM with Improved Read-SNM", in Proc. International Conference on Design \& Technology of Integrated Systems in Nanoscale Era, 2013, pp.35-38.

[7] P. Roche, J. M. Palau, C. Tavernier, G. Bruguier, R. Ecoffet, and J. Gasiot, "Determination of key parameters for SEU occurrence using 3-D full cell SRAM simulations," IEEE Trans. Nucl. Sci., vol. 46, no. 6, pp. 1354-1362, Dec. 1999. 
[8] J. M. Palau et al., "Device simulation study of the SEU sensitivity of SRAMs to internal ion tracks generated by nuclear reactions," IEEE Trans. Nucl. Sci., vol. 48, no. 2, pp. 225-231, Apr. 2001.

[9] Shah M. Jahinuzzaman, Mohammad Sharifkhani, and Manoj Sachdev, "An Analytical Model for Soft Error Critical Charge of Nanometric SRAMs", IEEE Trans. Very Large Scale Integr. (VLSI) Syst., vol.17, no.9, pp. 1187-1195, Sept. 2009.

[10] Naveen Verma and Anantha P. Chandrakasan, “A $256 \mathrm{~kb} 65 \mathrm{~nm}$ 8T Subthreshold SRAM Employing Sense-Amplifier Redundancy”, IEEE J. Solid-State Circuits, vol.43, no.1, pp.141-149, Jan. 2008.

[11]Tae-Hyoung Kim, Jason Liu, John Keane, and Chris H. Kim, “A 0.2 V, 480 kb Subthreshold SRAM With 1 k Cells Per Bitline for Ultra-Low-Voltage Computing”, IEEE J. Solid-State Circuits, vol.43, no.2, pp.518-529, Feb. 2008.

[12] Jinhui Chen, Lawrence T. Clark, and Tai-Hua Chen, “An Ultra-Low-Power Memory With a Subthreshold Power Supply Voltage”, IEEE J. Solid-State Circuits, vol.41, no. 10, pp.2344-2353, Oct. 2006.

[13]Ik Joon Chang, Jae-Joon Kim, Sang Phill Park, and Kaushik Roy, “A 32 kb 10T Sub-Threshold SRAM Array With Bit-Interleaving and Differential Read Scheme in 90 nm CMOS”, IEEE J. Solid-State Circuits,vol.44, no.2, pp.650-658, Feb. 2009.

[14]Ming-Hsien Tu, et al., “A Single-Ended Disturb-Free 9T Subthreshold SRAM With Cross-Point Data-Aware Write Word-Line Structure, Negative Bit-Line, and Adaptive Read Operation Timing Tracing”, IEEE J. Solid-State Circuits, vol.47, no.6, pp.1469-1482, June. 2012. 
[15] Yi-Wei Chiu, et al., “40 nm Bit-Interleaving 12T Subthreshold SRAM With Data-Aware Write-Assist”, IEEE Trans. Circuits Syst. I, Reg. Papers, vol.61, no.9, pp.2578-2585, Sept. 2014.

[16]T. Calin, M. Nicolaidis, and R. Velazco, "Upset Hardened Memory Design for Submicron CMOS Technology", IEEE Trans. Nucl. Sci., vol.43, no.6, pp.2874-2878, Dec. 1996.

[17] Shah M. Jahinuzzaman, David J. Rennie, and Manoj Sachdev, “A Soft Error Tolerant 10T SRAM Bit-Cell With Differential Read Capability", IEEE Trans. Nucl. Sci., vol.56, no.6, pp.3768-3773, Dec. 2009.

[18] Guohe Zhang, Jun Shao, Feng Liang, and Dongxuan Bao, “A novel single event upset hardened CMOS SRAM cell”, IEICE Electronics Express, vol.9, no.3, pp.140-145, Feb. 2012.

[19]P. Hazucha, T. Karnik, J. Maiz, S. Walstra, B. Bloechel, J. Tschanz, G. Dermer, S. Hareland, P. Armstrong, and S. Borkar, "Neutron Soft Error Rate Measurements in a 90-nm CMOS Process and Scaling Trends in SRAM from 0.25-mm to 90-nm Generation,” in Proc. IEDM Tech. Dig., Dec. 2003, pp. 21.5.1-21.5.4.

[20]Hiroshi Fuketa, Masanori Hashimoto, Yukio Mitsuyama, and Takao Onoye, “Alpha-Particle-Induced Soft Errors and Multiple Cell Upsets in 65-nm 10T Subthreshold SRAM,” in Proc. IEEE Int. Reliab. Phys. Symp., May. 2010, pp. 213-217.

[21]Hiroshi Fuketa, Masanori Hashimoto, Yukio Mitsuyama, and Takao Onoye, "Neutron-Induced Soft Errors and Multiple Cell Upsets in 65-nm 10T Subthreshold SRAM,”IEEE Trans. Nucl. Sci., vol.58, no.4, pp. 2097- 2102, Aug. 2011. 
[22]Paul E. Dodd and Lloyd W. Massengill, "Basic Mechanisms and Modeling of Single-Event Upset in Digital Microelectronics,” IEEE Trans. Nucl. Sci., vol.50, no.3, pp. 583-602, Jun. 2003.

[23] Jan M. Rabaey, Anantha Chandrakasan, Borivoje Nikolic. "Digital Integrated Circuits-A Design Perspective, Second Edition.” 658-661.

[24]Neil H. E. Weste, David Money Harris. "Integrated Circuit Design, Fourth Edition." 458-460.

[25]Leland Chang, Robert K. Montoye, Yutaka Nakamura etc. “ An 8T-SRAM for Variability Tolerance and Low-Voltage Operation in High-Performance Caches," IEEE Journal of Solid-state circuit, vol.43, no.4, Apr 2008.

[26] Y.Morita et al., "A Vth-variation-tolerant SRAM with 0.3-V minimum operation voltage for memory-rich SoC under DVS environment," Proc. VLSI Circuits Symp., 2006, pp. $13-14$

[27]K.Zhang et al., “A 2-GHz 70-mb SRAM in 65-nm CMOS technology with integrated column-based dynamic power supply,” JSSC, vol.41, no. 1, Jan. 2006, pp. 146-151.

[28]T. Kim, H. Eom, J. Keane, and C. Kim, "Utilizing reverse short channel effect for optimal subthreshold circuit design," in Proc. Int. Symp. Low Power Electronics (ISLPED’06), Oct. 2006, pp. 127-130.

[29]Robert C. Baumann. "Radiation-Induced Soft Errors in Advanced Semiconductor Technologies," IEEE Trans. On Device and Materials Reliability, Vol. 5, NO. 3, Sep. 2005, pp. 305-316. 
[30] J. Bisgrove, J. Lynch, P. McNulty, W. Abdel-Kader, V. Kletnieks and W. Kolasinski, “Comparison of Soft Errors Induced By Heavy Ions And Protons,” IEEE Transactions onNuclear Science, Vol. NS-33, No. 6, pp. 1571-1576, Dec. 1986

[31]J. S. Fu, C. L. Axness and H. T. Weaver, "Memory SEU simulations using 2-D transportcalculations,” IEEE Electron Dev. Lett., Vol. EDL-6, No. 8, pp. 422-424, 1985

[32]C.L. Axness, H. T. Weaver, and J. S. Fu, "Mechanisms Leading to Single Event Upset,’IEEE Transactions on Nuclear Science, Vol. NS-33, No. 6, pp. 1577-1580, 1986

[33] J. A. Zoutendyk, L. S. Smith, G. A. Soli and R. Y. Lo, "Experimental Evidence For a NewSingle-Event Upset (SEU) Model in a CMOS SRAM Obtained From Model Verification,'IEEE Transactions on Nuclear Science, Vol. NS-34, No. 6, pp. 1292-1299, 1987

[34]R. L. Woodruff and P. J. Rudeck, "Three-Dimensional Numerical Simulation of SingleEvent Upset of an SRAM Cell," IEEE Transactions on Nuclear Science, Vol. 40, No. 6, pp.1795-1803, 1993

[35]P. E. Dodd and L. W. Massengill, "Basic Mechanisms and Modeling of Single-Event Upset in Digital Microelectronics," IEEE Transactions on Nuclear Science, Vol. 50, No. 3, pp. 583-602, Jun. 2003

[36]P. E. Dodd, F. W. Sexton, G. L. Hash, M. R. Shaneyfelt, B. L. Draper, A. J. Farino, and R. S. Flores, "Impact of technology trends on SEU in CMOS SRAMs," IEEE Trans. Nucl. Sci., vol. 43, pp. 2797-2804, Dec. 1996.

[37]H. T. Weaver, C. L. Axness, J. D. McBrayer, J. S. Browning, J. S. Fu, A. Ochoa1, Jr., R. Koga, “An SEU Tolerant Memory Cell Derived From Fundamental Studies of SEU 
Mechanisms in SRAM," IEEE Transactions on Nuclear Science, Vol. NS-34, No. 6, pp.1281-1286, Dec. 1987

[38] Shah M. Jahinuzzaman, David J. Rennie, and Manoj Sachdev, "A Soft Error Tolerant 10T SRAM Bit-Cell With Differential Read Capability," IEEE Transactions on Nuclear Science, Vol. 56, No. 6, pp. 3768-3773, Dec. 2009

[39] S. Jagannathan, T. D. Loveless, B. L. Bhuva, S.-J. Wen, R. Wong, M. Sachdev, D. Rennie, and L. W. Massengill, "Single-Event Tolerant Flip-Flop Design in 40-nm Bulk CMOS Technology”, IEEE Trans. Nucl. Sci., vol.58, no.6, pp.3033-3037, Dec. 2011.

[40]Kuande Wang. "Ultra Low-Power Fault-Tolerant SRAM Design in 90nm CMOS Technology." Thesis for Master of Science, University of Saskatoon, 2010, [41]Sven Lütkemeier, Ulrich Rückert. "A Subthreshold to Above-Threshold Level Shifter Comprising a Wilson Current Mirror", IEEE TRANSACTIONS ON CIRCUITS AND SYSTEMS-II: EXPRESS BRIEFS, VOL. 57, NO. 9, pp. 721-724, SEPTEMBER 2010. 